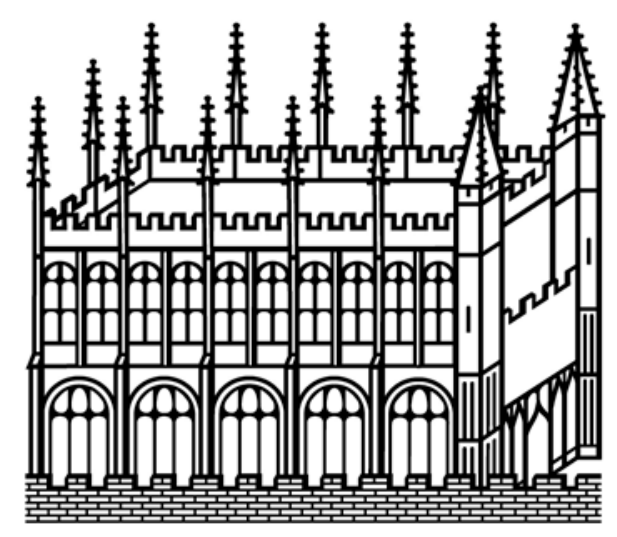

\title{
Bodleian Libraries
}

\section{UNIVERSITY OF OXFOR D}

This book is part of the collection held by the Bodleian Libraries and scanned by Google, Inc. for the Google Books Library Project.

For more information see:

http://www.bodleian.ox.ac.uk/dbooks

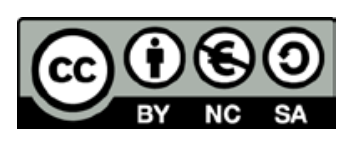

This work is licensed under a Creative Commons Attribution-NonCommercialShareAlike 2.0 UK: England \& Wales (CC BY-NC-SA 2.0) licence. 


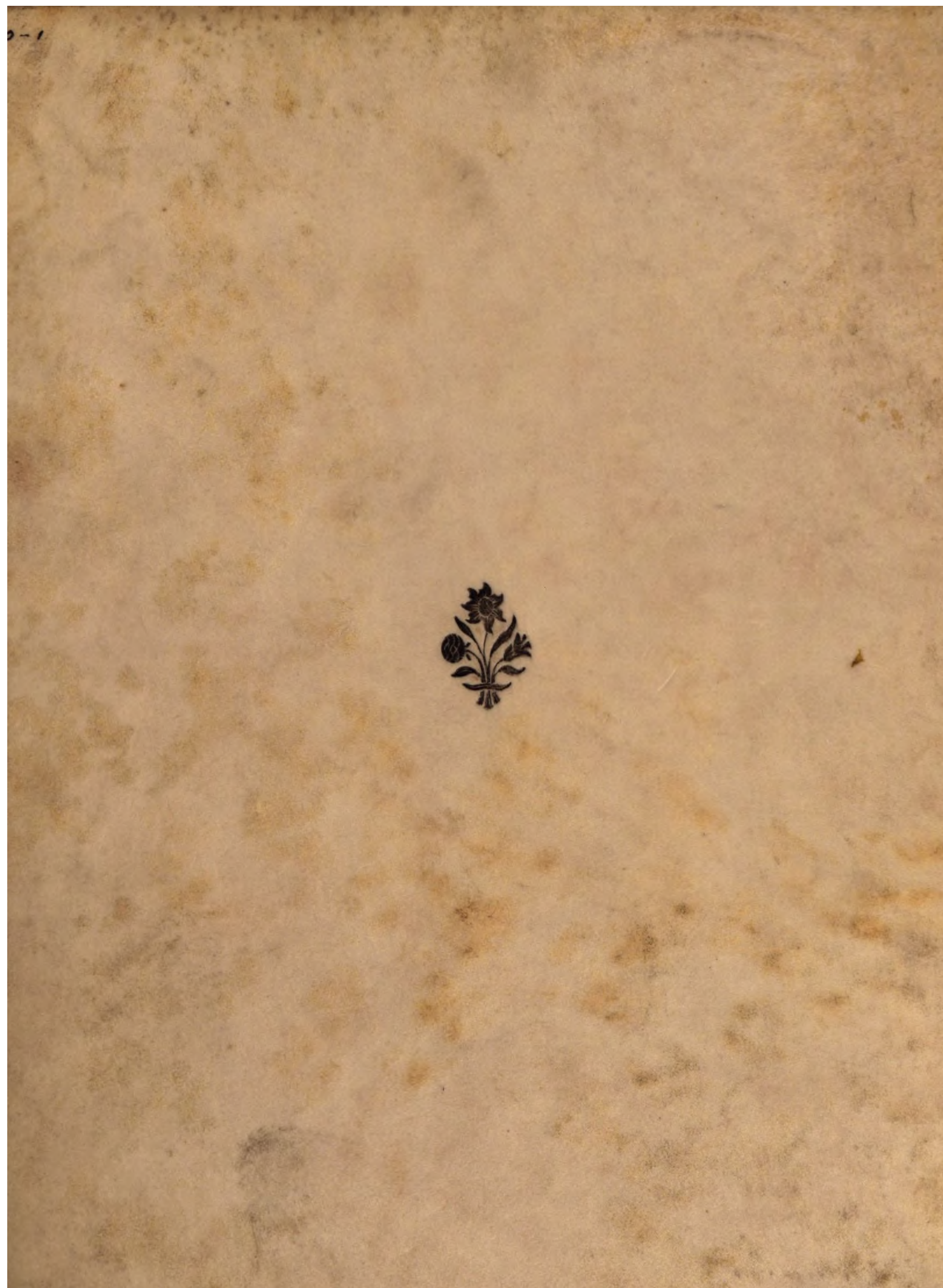





$\checkmark$ 


$$
\text { ㅈ... }
$$




\section{feterbael ban de bíer ertife \\ SCHIP-V AERDEN Der}

Hollandtfche en Zeeuwfche SCHEPEN, N A A R

N O V A Z E M B L A,

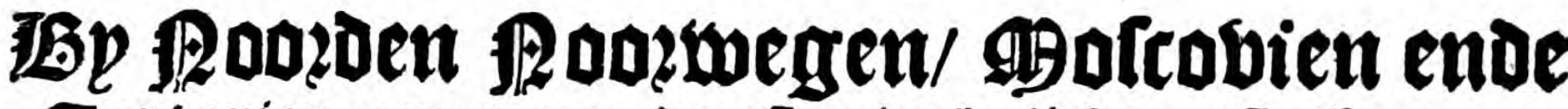
ILartarien om / na de Coninckrijcken Cathap en Cbina. untgevaren in de Faren I 594. 1 595. 1 596. en 1609. ende

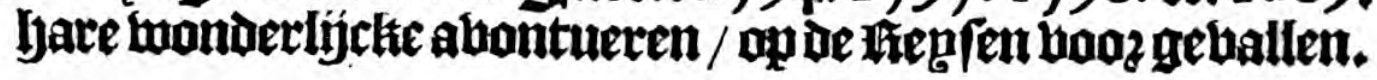
Denlaetfen Druck vannieuzvs overffen, en met fchoone Figueren verbetert.

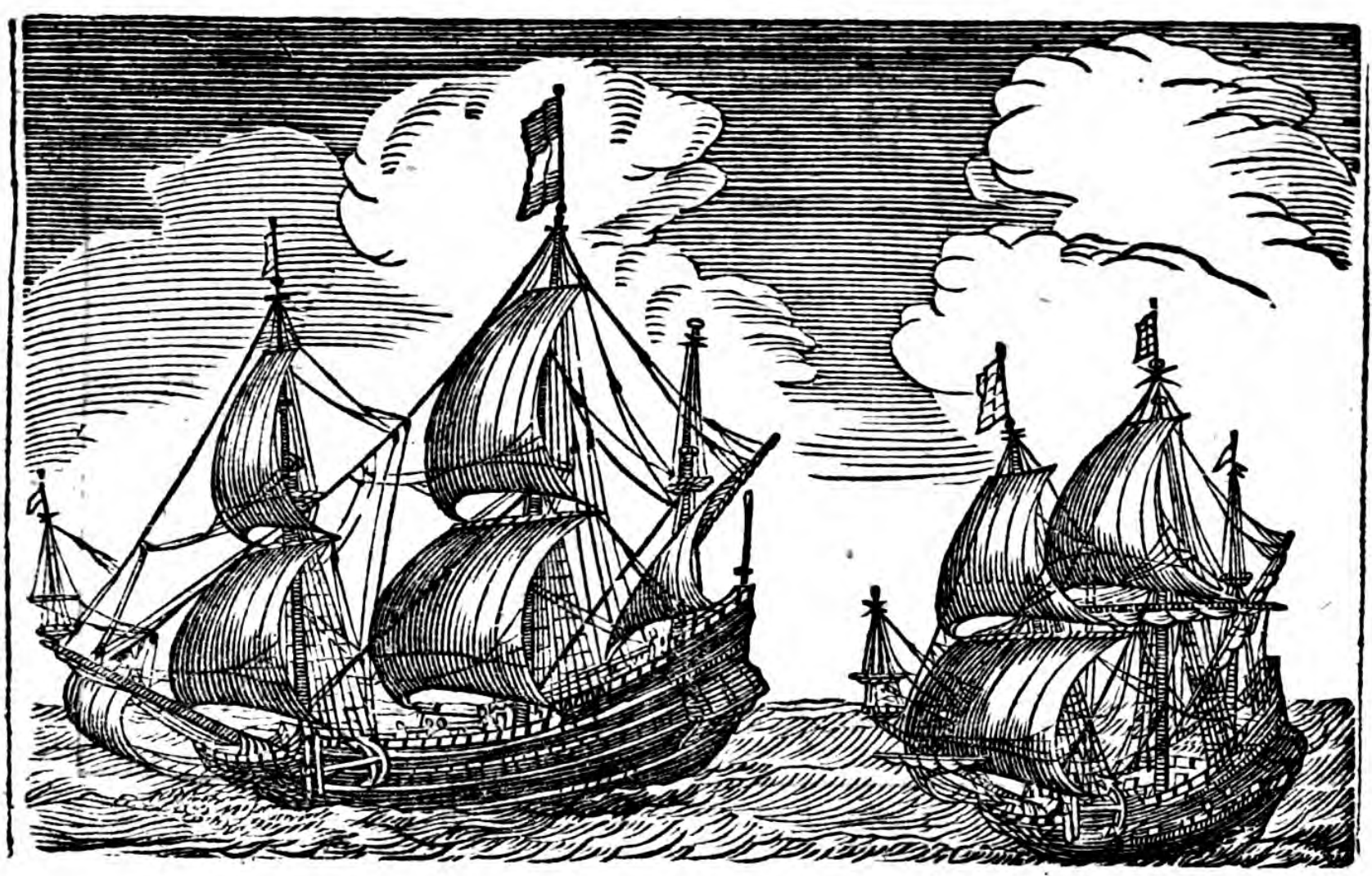

T'AMSTERDAM, 203. f 332

Gedquckt bp GILL Is JoOs TEN SA E G H MAN, Bbeck-Dzucher

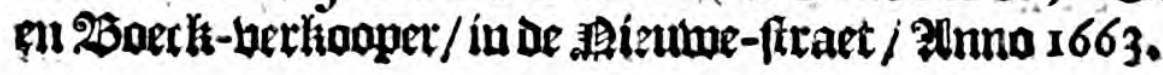




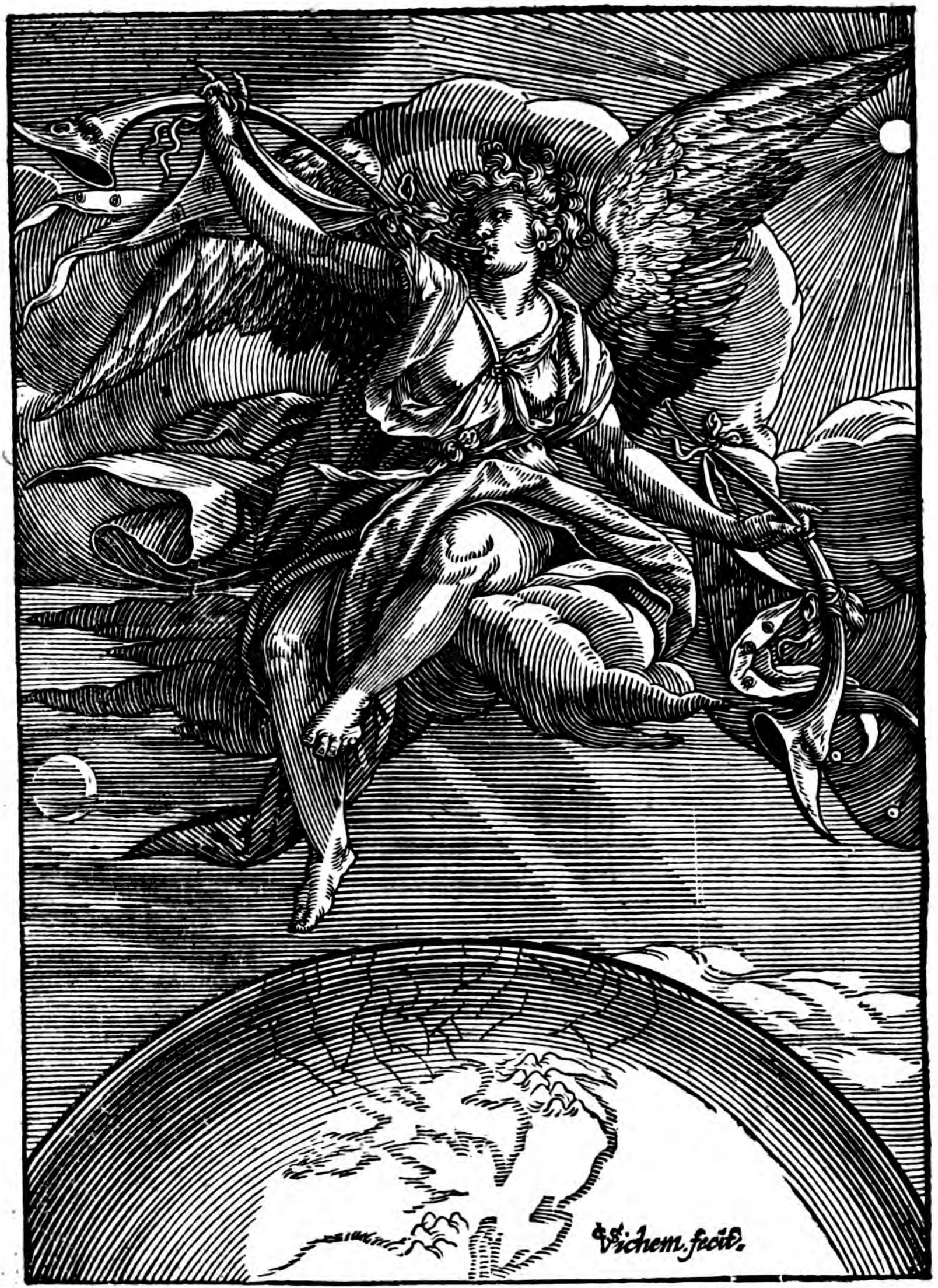

Tk roon u hier in Druck roem-waerde Batavieren, De ftreecken van ' $t$ Compas, en wat men al voor Dieren Vindt in het koude Noord'? ick wijs u meê de Re'en Waer dat men veyligh leydt in noodt van quade Zeên. Ick ftel 't u pertinent, ick noemt u met de Naem; Daerom en koopter geen dan met dees vlugge $F_{A} \mathrm{E}_{\mathrm{M}}$. 


\section{Kort Verhael vand' eerfte Schip- vaert der Hollandtfche ende Zeeufche} Schepen, na Nova Zembla, by Noorden Noorwegen, Mofcovien ende Tartarien om, na de Coninghrijcken van Cathay ende China, uytgevaren in den Jare I 594. Getrocken uyt het fournael van Gerrit de Veer.

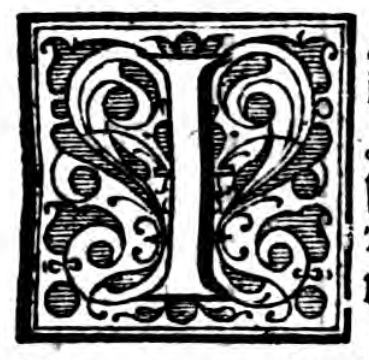

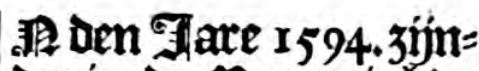
Det in de Beremighioe Fetriarlanden/tēn ber= foeke ban eenen BalthaZar Moucheron 隹oop= man / wounende tat

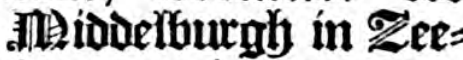
lanot / ente fijn Compagnie / met com=

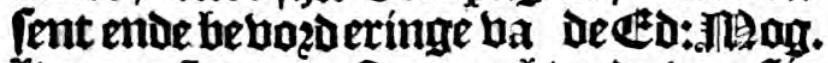
*eren Staten Benerarl/enie ban fin

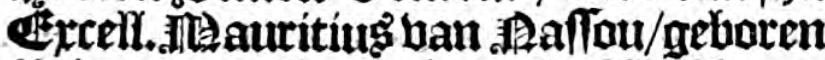

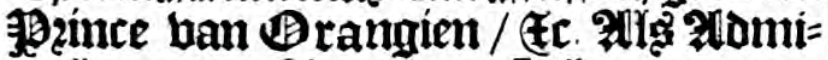
rael ban der \%ee / Defue Srbepen toege= maeckt/een tot \$imfterdam/een in ₹ere lanot/ ende een tat Gincfiljumfen/am op te baen de gelegenthepot ente frectínge nae de landen ende coningbrijcken ban Cathay ente China, by Jooguen Noorwegen, Mofcovien ende Tartarien am.

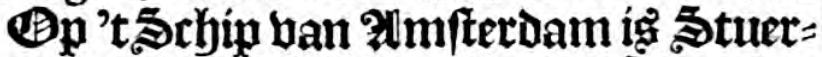
man gebeeft MBillem 2 barent/5 ban der Scljellíng/25ozger der felber Staut/een feet filoerf / bermatert / moe bedreben man in de tanft ban de \%ee-bart / bu fem febtende een Scljellinger Bifferter Slacht / om lom in fín booghtjenomen repfe (bp aloien fy ban d' andere thee feljepen quame te frbeuoen) gefelfichap te jouben.

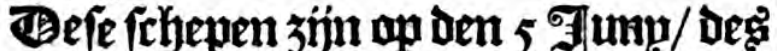

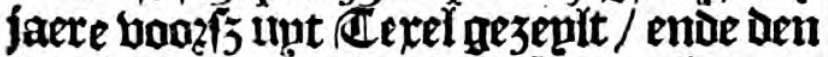
23. Dita met gaeden boappoet grekamen

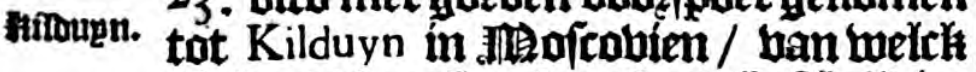

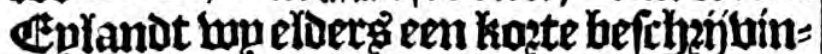
ghe fulfen ftelfen/ Opg sen 4 Glung gepl=

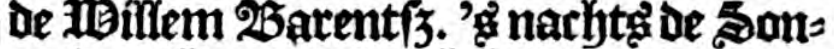
ne doen hy op 't laeghfte was/ te weten

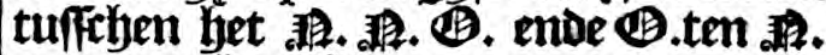
ende bont hem felben op 73. mrad. 25.min joolz fooghte. Dit gefchiebe ontrent 5.0ft6.mijlen ban't lant genaemt Nova Sembla: Jaet na benoen in't ooftwaet ober / ende quamen na 5. mijlen zeplente by een lage untfeltende boect / Die fu noemien Langenes : Therht aen Defen xat boeck na.'t ooften wateell groote bag/ albaer fin met de boot aen fant boeren/ maer en bonden geen bolels. Tufichen Capo Baxo, dat 4. mílen uall Langenes lepot ende de luefthoecti ban Loms-bay, waren twee intuíctien.

Loms-bay íg een groote bujfor 2 ban / ? ende beeft aen de weftzíde een fobone yaben 6.7 .8 .badem diep/Daer boeten=

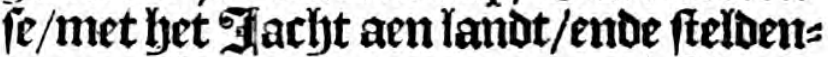
oer een baken ban een oude maft die fi daer bonien. Jefe Baen noembene Loms-bay ban megen eenerlep aett ban xon bogelen alfo genoemt / Diefe baex in grod= oft th te menighte bonden/wefenie gront ban pege licljaem / maer fo hlepn ban bleugeld Dat het wonder íg / boe die filenne bleus gelg Dat fware líchaem Dzagen fommen. Sn befben lyaer neften op fteple bergen/ om ban de beeften befajermt te jijn/ bzoeden maet een ep teffenga / ente jín booz oe meniclien fo mepnigh bebeeft/ Dat al gríjptmen oer eeníge op lyaer neft/ fo en bliegen noclytans de andere daer ontrent niet weght. 
ittepar

Dam Loms-baey zepldené nae het anderen mozp maer 6. ende ftract daet

Egla:k.

Admiraliteyts Evlandt, 't welck aen de na weder 10,1 1.1 2. Ten 6. Fulu/aly de ooft-zíjoe niet feljoon maer berre black Som Jaozon was / quamenife met tis / ende daeroin een groot ftuck weegg fichoon weder neffeng de Swarten-hoeck, hos moet gef hout zijn/ te meer om dat het leggende op 75.grad. 20. min.en ontrent

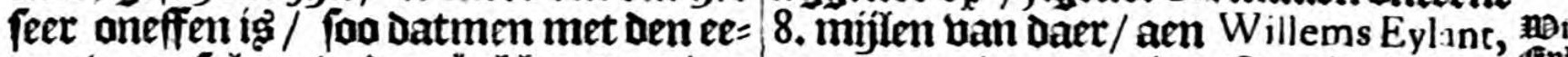

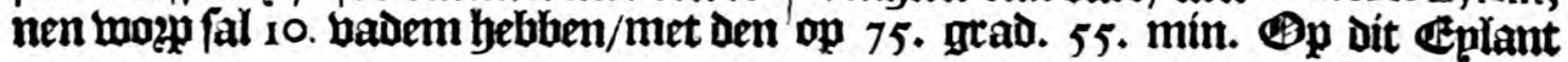
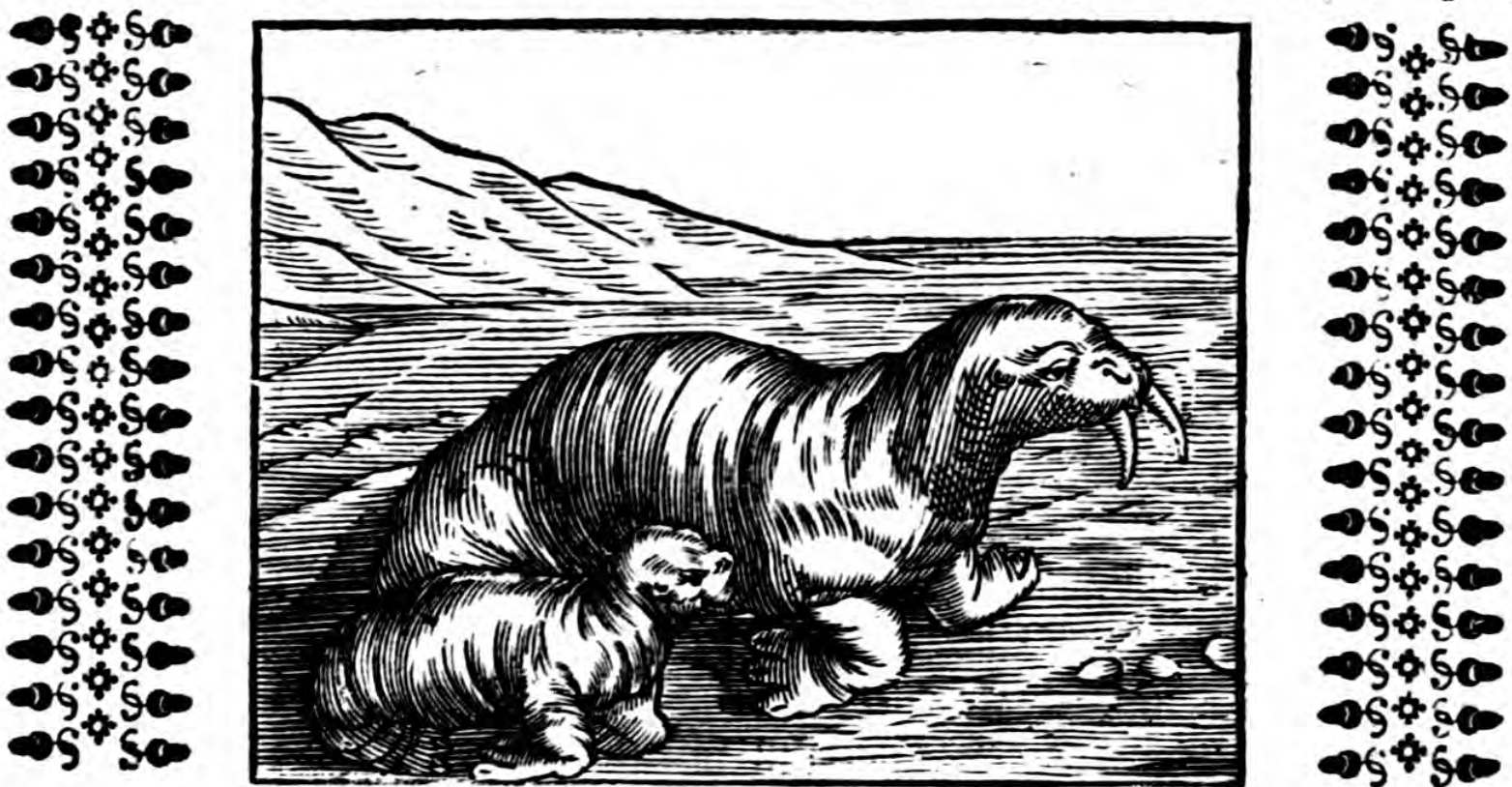

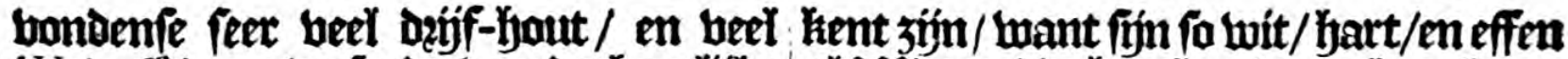

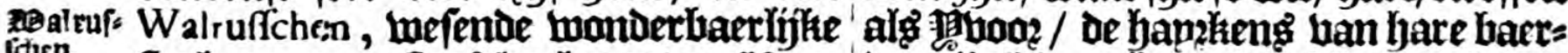
fithen.

fterhe zer-monfter:s/ueet grooter algen Den zín filepue horenkeng//bp Kange eben

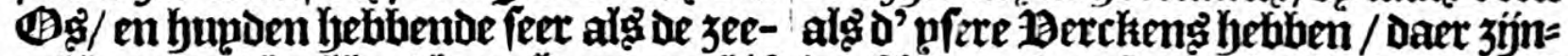

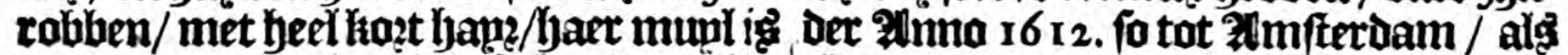
Der leeturen mupl gelijk/ph boudent dik= eldergi in follant / twee te fien getweeft /

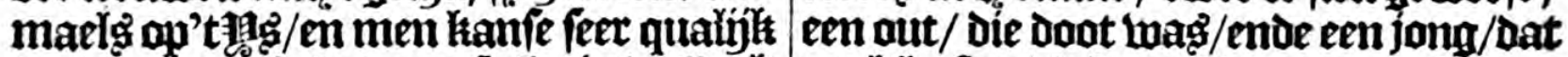

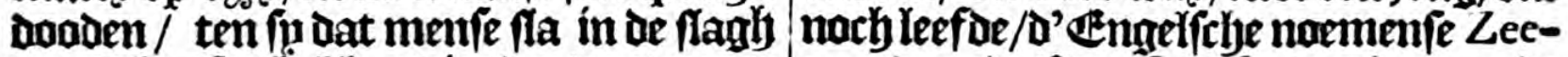
ban't hooft / hebben bier boeten / maet geen ouren / beengen maer een of twee fongen boot en als de Diffelyets baer op

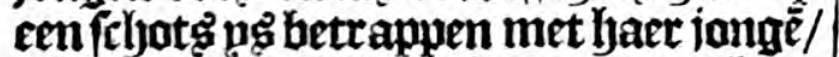
fon werpenfe de felbe jongen boo baet in't water/ nemenfe in haer armen / en bompelen daer mede op en neder / en alf = in fe haer aen de fehumten willen lweeken / maer de 2 ber dies niet tegenftaende be= ofte te were ftellen/fo werpenfélyaer jon=, wergeen wonderbaerlijclie ktarht/ dier=

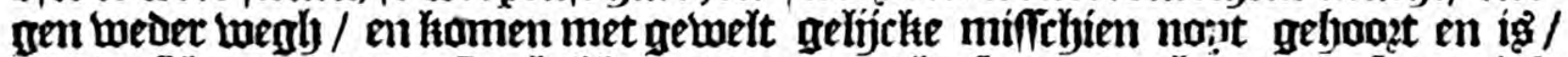
nae de felyuten toe. Sop hebben twee want lyp fpeang nach ap/enoe nwom in't

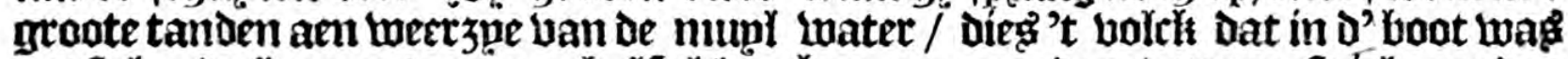
intftekende/lang ontrent een half el/ $\mathrm{de}=\mathrm{h}$ em nae roepden / en een firich om den

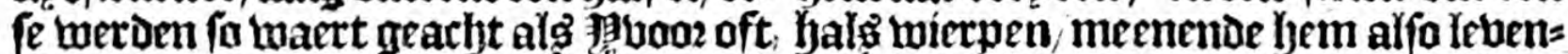

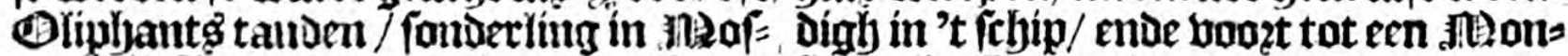
robien/ Iartarien / cu elderge daer fe be=, fter in 

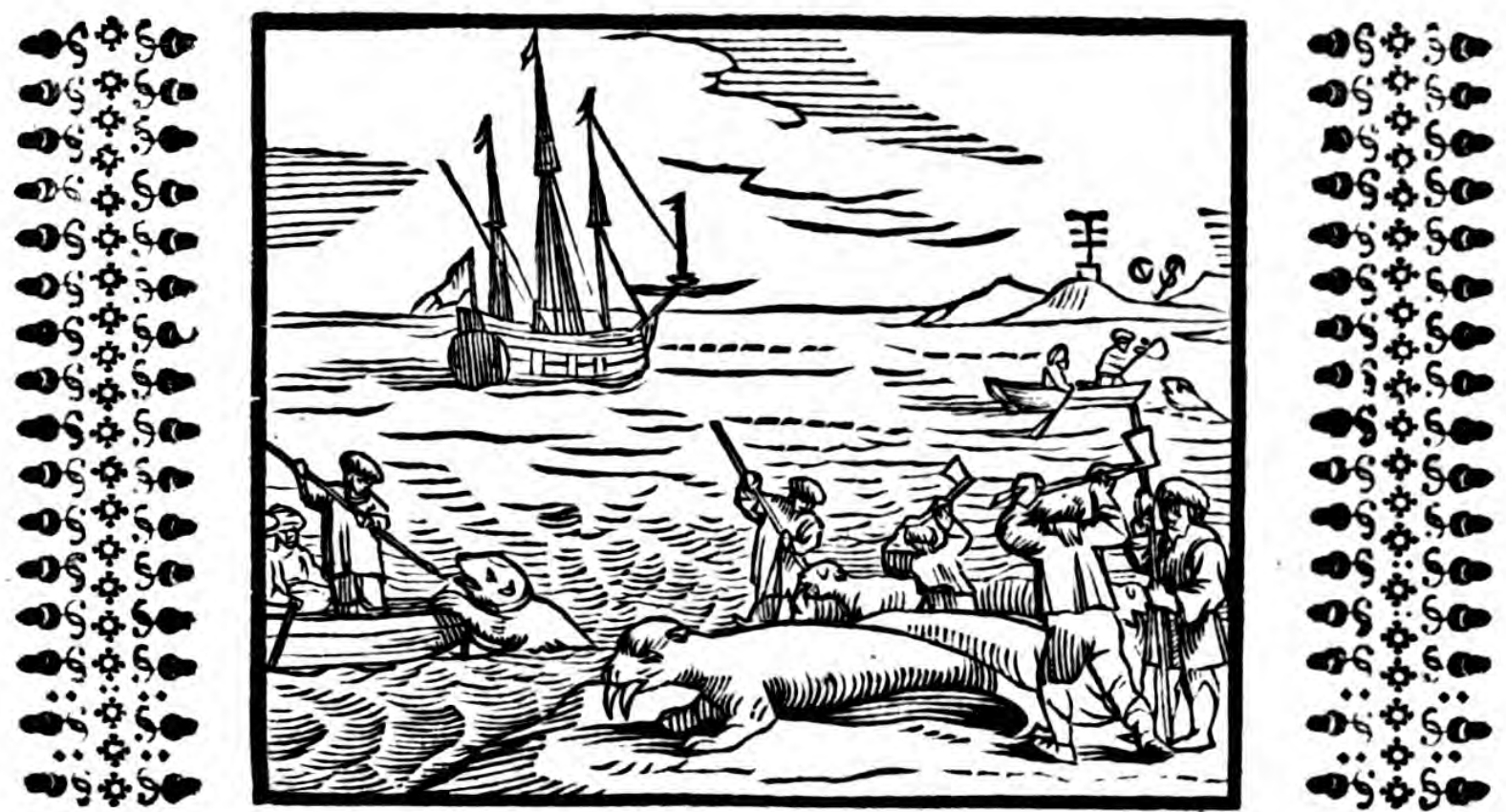

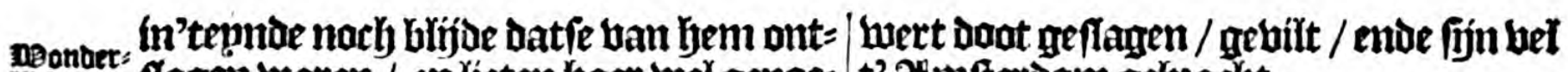

lijthe

trache ban 2 geet. flagen waren / en líeten faer wel genae = $t^{\prime}$ \#mfterdam gehzacht.

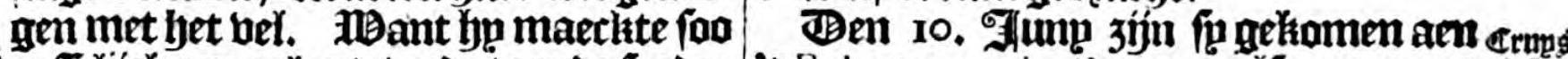

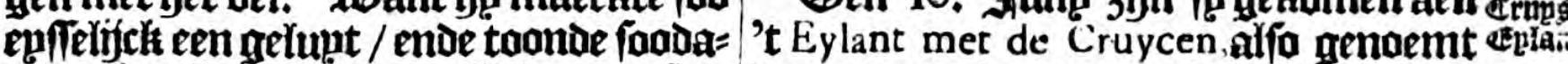
nigen kraclyt/Dat het qualijek om te feg= wam 2. groote Grupern die daer op fton= gen / ende noch min boos den onerbare $=$ den/en leggende ontrent 2.groote mílen

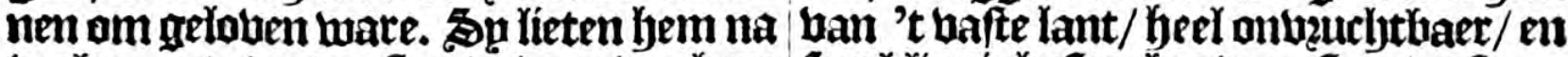
beel moepte wat ruften/ende gaben bem feer Klippigh/ftreckende ooft en weft on= wat meer bots met het toutw dat hy om trent een balf mijl langly /met een rif op Den hals hadde / flepende ljem foo ban elef eunoe. Bntrent 8 . mílen ban daer langer hant na/om hem mat te makken/ Iepht ien Hoeck van Naffouwen op 76. rouw. ende DBiflem Sarent/3. palmoe bem al- grad. zijnde een lange en blacke te-met wat aen / maer de 2 beet f wom diemen beg feljoulwen moet / want daet aen 't hoot/en floegly fín poten daet ach= wag dzoogte ban 7. vadem feer berte ban

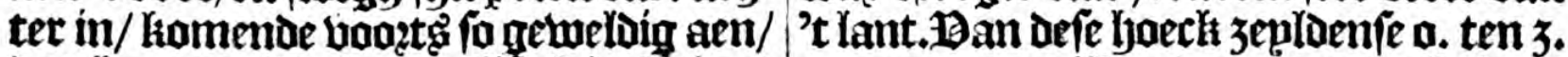
Dat ho met fín lyalbe líf al in 't boot en a. 3.0. 5. mílen / en doen oocht lyaet

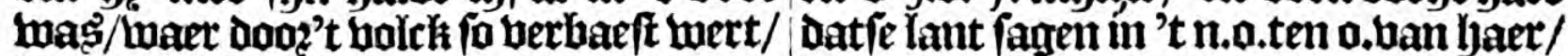
datten fp bluchten ban acliteren't boot daet fp nae toe zeploen/mepnende't was naer booren/ mepnende andergs niet dan een anoer lant dat nooztwaert ban NoDat palle lijueloog waren/Docly werden va Zembla lagh/maer't began fon ftíf noch Doo een fonderlinge abontuer ber = te waepen / Dat fe fracx affe de zenlen in loft / mitg de frrick die de 2 Seer am den nemen moeften/ende de 3 ee gingh fo lyol/

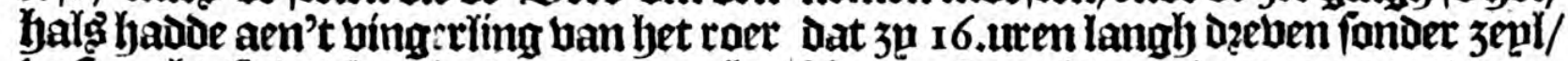

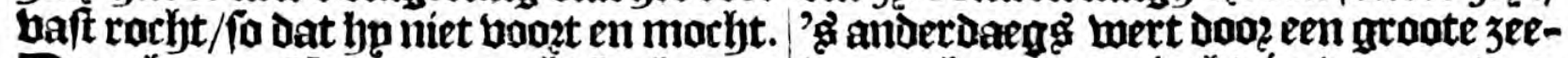
Toen lyg nu alfoo in onmarbt lagh/ver- water haer roep-jacljt in be gront ge: ftoute fif een ban't volfi/ende ftack hem fmeten/alipo dat ín't vetlooren/en noch met een fyalue lancie dat ly ban't boot langly fander 3 enl dzenen/ tot dat fo met neder te water biel / roevende fo te met de 3. w. Son oicht aen 't lant ban Nova bost nae't fethip moe hem naeflepende/ Sembla quamen. Den r3. Flimp ont=

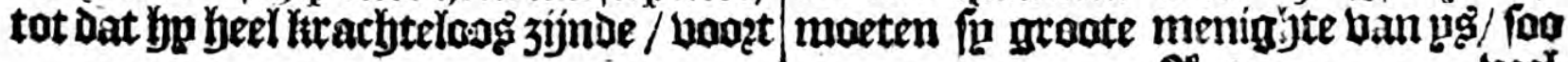




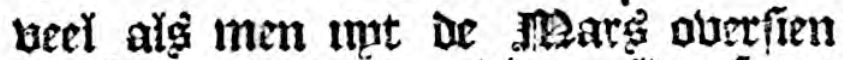
martit / ende den r4. Uíto zepliden pa tot be boogte vall $77^{\frac{1}{2}}$.grad. ch quamen boen weder aen een effen velt pa/ po groot dat= men 't niet ouerfien kande. Den 19. 'gi mos gheng quamen fo weder dicht by't lant van Noya Sembla ontrent de vaoef Capo de Capo de Naffou, ende den 26. aen den Eraoft. Capo de Trooft. Den 29. Díta bnaen fux haer op 77. graden / ende toen lagly de allernoazdelijeftite hoek ban Nova Semmogect. bla, genaemt Y fhoeck, reefht onft ban haer. Taer bondenfe eeníglye fteentjeng die glinfterden alg gaut/ende naemben

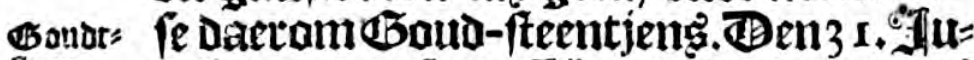
fteen. lu laberden ín tuffechen't landt ende pge betwen met lieffelijét ftil weder / ende En!an: quamen aen de Eylanden van Orangien, ten tan onder anderen/aen cen/op welche fipon yien.

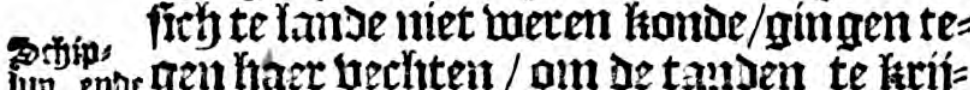

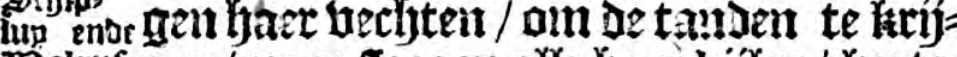
10almur: ren/maer flaegen alle haer bijlen/ Ytazte = ferberpot. Yaffen / ende fuieffen daer ap in ftuctien fonder een daet uan doat te flaen/ dam dat ju'er eenen een tandt unt floegen/Die fu mede namen. Siende dam datie met becliten niet op haer wimnen fanden / fa Gefloteníe t' fichep te baren om grof ge= felyut te halen / maex lyet lieftont fo feer te haauen / ende't po aen groate ftucken te feheuten / Dat fir't naelaten moeften. Op de felve tijt lromben fe aock een quan= te witte 2 ber flapende / en feljoten ljem in fín lumt / maer hy liep noch weady / ende begaf hem in't water / Doch lyet

2heer boobt ce flagen. bolck raevie hem met bet jacht na/flae= gen ljem boast doot / enoe feepten lyem op't ngs / daex in fyem aen een lyalue lan= rie bafi maeckten / en menndenfe te ko= men balen/ dan muluín ljet boe langlyer boe lyarder begon te wamen / ende lyet pge te foheuren / foo is daer mede niet lam gevallen.

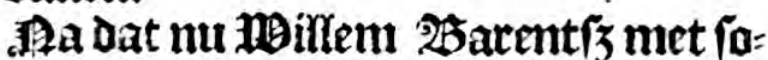
Danigly abontuer alg uethaelt ig / tot de
Eslanden van Orangien gehonten wags / eribe nae alle gesame arbept bentain/

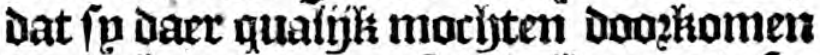
am de begannen repfe te baibaeren/ ofte 't lant boozder ap te doen / ende 't bolck oock beftandt berdacietígly te wozden/ foa werdat het boos goet aengefien dat/e we= derom fouden fieeren / am alfo bu de an= orr Brbepen te flomen die na de Waygats ofte Straet de Naffou, yaer courge ano= men badden/ende te bernemen wat ope= nínge die aldaer gebonden badoen.

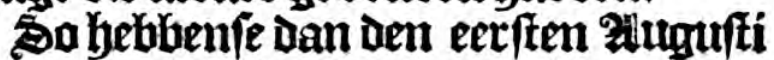
haer courgi weder te rumge gewent/ende jún woag bn den Y hoek, Capo de Trooft, Capo de Naffou, ende andere baecten meer / op den 8. Dito geftomen aen een laeg hlepn Eplamdelten leggende op een baif mijl aen't lanot /'t welck ip noem= Dent het Swarte Eylandt, om dat het bo= ven fwat wag. Taer peploe IBiflem 25 arent $\left\{3\right.$ de hoogte deg pools op $71 \frac{1}{2} \cdot$ gr . en daer was een mooten inwoich / 't wel= He HBiflem 2Barent/3 gifte de plat te wefen daet Glíbier 2 Benmel te boren ge= weeft hadde/genaemtConftintfarg. Bas míl'ten ban 't Swarte Eylandt boniuení noctyeen andere fiechte hoech / daer een crunga ap ftant/ende noemben bem daet: am de Cruyfhoeck. Bam Daer zenloenfe hu de wal Yangsi 4. mílen/ ende quamen ${ }^{\text {bo }}$ Doen aen een andere flechte boeck / Daer achter een gronten invónch was / die naemden in den vijfoen lyoeck / ofte S.Laurens-hoek. Degn mílen boozder lent de Schans-boeck, met een laghe pwartere Filip dielft aen't lanot/Daer een fitupgs op faet. Hier boerenfe met het boot aen be lant/ende bernamen datter bolf geweeft was / Die om lyarent wille geblucht wa: ten / want fu bonden aldaer feg factien met fioggen-med brgraben / ende een fteenhoop bp 't krupgs. Een grootelínex itjoot van daex ftondt noch een fatups met depe hup/en ban bout gemaecht op

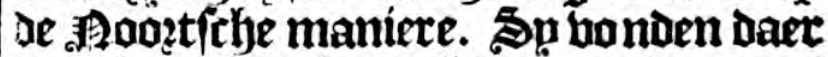
oackín íne fupfen veel tamen-dungen / waer wot fa betmoeden datter eenige 


\section{Om't Noorden na China.}

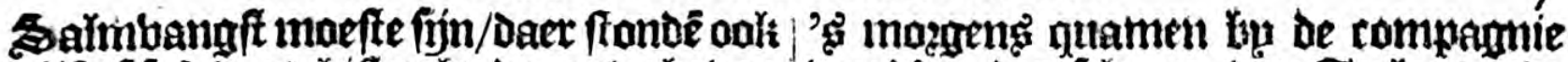

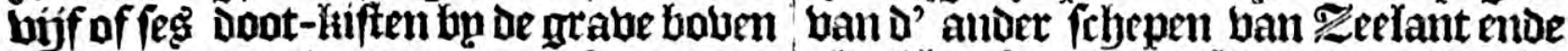

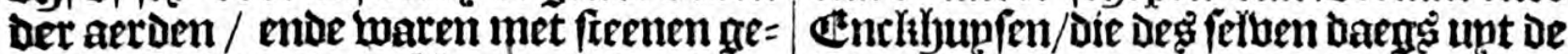

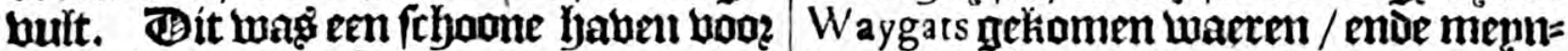

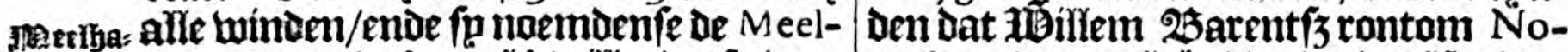
ben. haven, om deg meeld wilfe Datfe Daer bonien. Fuffelyen de Meelhaven ende de 7. Schans-hoeck leut De Baeye van S. Laul-

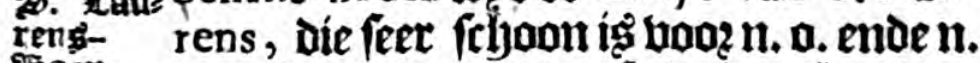

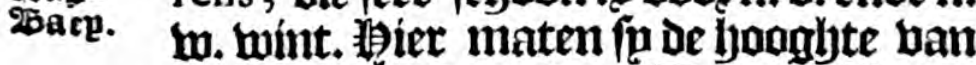
be son ap $70 \frac{1}{2}$. graden. Then 12 . Flugu=

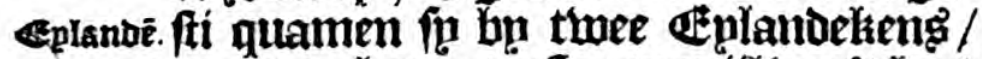
dare ban lyet unterfte een mujl ban't lant D. Clara lagly / Defe noemdefée. Clara. Jen 15 . di= to 3. w. Son heeft Millem $25 a$ atent 13 ge $=$ meten dega jooolz bougte ap 69. maden 15. min. ende doen gingenfe nocly 2. mity=

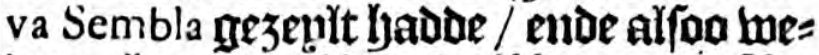
der wefeert unaj Dooz de Weygats. 23u malktanderen komentoe dedenfe tephenga ban blijfrchap met eerfelyoten ende ans Dersis / bertellende elck anderen waer já al geweeft waren/ ende wat elck bezenft ende apgedaen ljadde.

TBit gedaen zimoe / bebbenfe haer rours mederom naet huys getwendt/en=

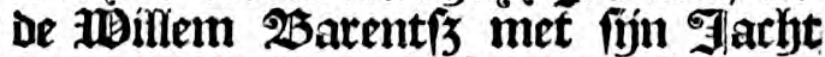
quamen op den 16. Septembeis booz Theterdam / mede bzengende een THal=

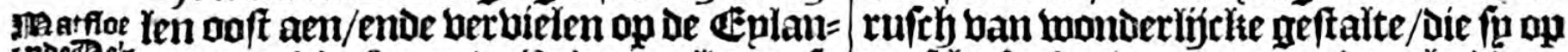
goe.

Kort Verhael van de tweede Schip-vaert by deHollantfche ende Zeeufche Schepen, gedaen Anno 1 595. achter Noorwegen, Mofcovien ende Tartarien om, naer Cathay ende China.

Medegetrocken uyt bet Fournael van Gerrit de Veer.
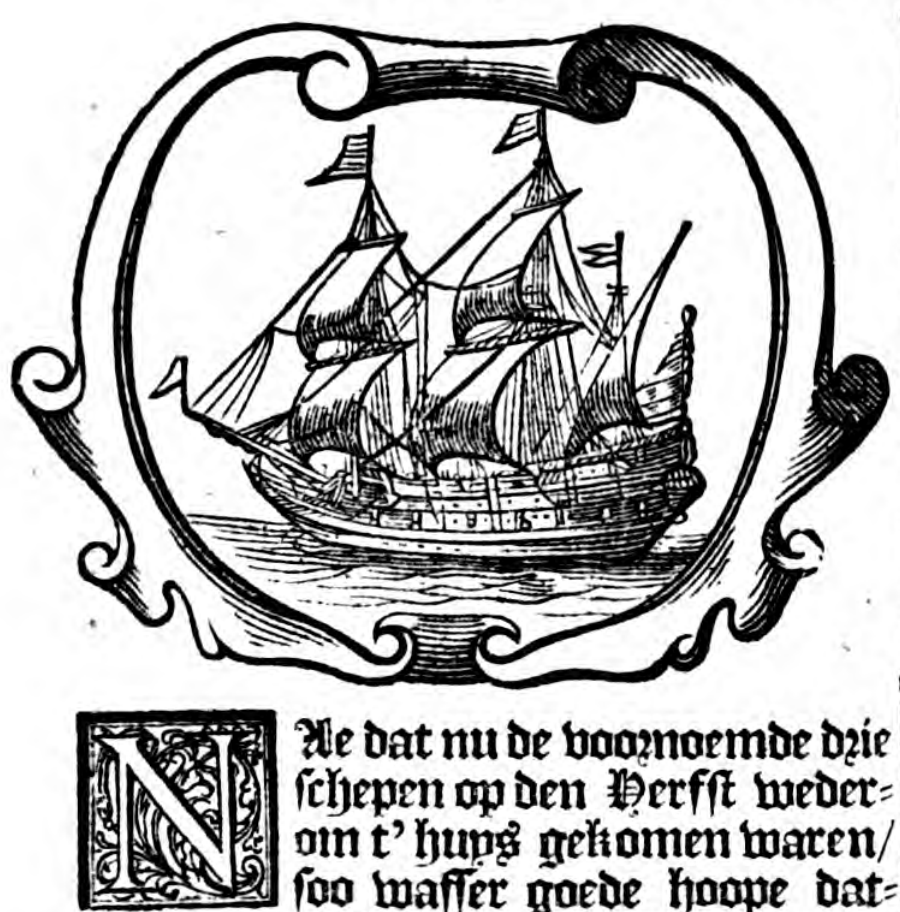

He dat nu de baomarmbe dzie ichepen op den Werfit weder= oun t' hupg teltomen waten foo waffer yoede happe dat

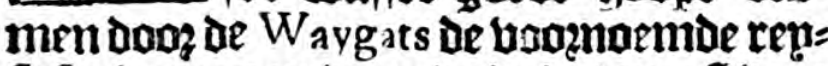
fe foude inogen inen/ende dat meeft boo? 't berfhalen Lan' $\mathrm{t}$ Zeeurche ende't đent=

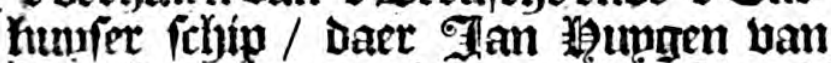

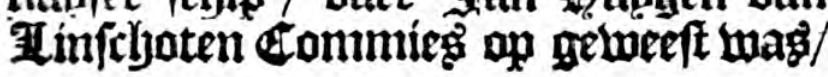

Die De fakie begn wat byeed boot-geftelt

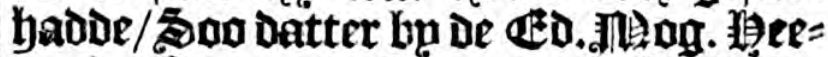

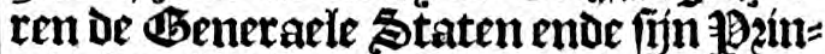
refirke Extellentir. hrefioten wert tegen 't boozjaer hederom eenitge fetbepen toe te maken / om niet alleenilich / ald te bo: ren / de baert op te doen ende te befipie= den/ maer ook eenige waren ende koop: man/chappen derwaert te te fenden/Daex in De kooplupden mortjten frbepen foo: Danig goet ; ale 't haer belief de / metee = nige $\mathbb{C}$ ommifen om 't felve te verbandes Ien ter plaetfe daer fu aen-Komen fou= Den/ende dit alleg bzant-ende litent-byg.

Petrus Plancius, een wermaert $\mathbb{C o f m a =}$ graphys ofte 1Berelt-fethýyer/ is mede een peincipael helender ende bebozderaet ban defe Sclhip-baerdt getweeft / die de peincipale ozoze van de caurfen trozói= ncert beeft webadt/alg bock be ftreckin= ge van de Kanden van Tartarien, Cathay, enveChina, maer wat ban de felbe te oos-

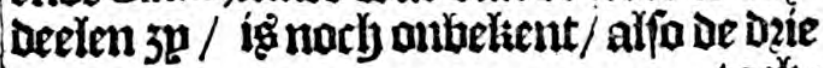
tact): 
'tochten sart om gedaen tot geenen ge= wenityten eunioe gyjeliamen / noch de courfeu bu ljem gheftelt badiomentyick achter-bolght pijn/ ende dat doos eenighe baoz-geuallen inconvenienten / díe boo? de kaztlyent deg tijot niet en kolnden berbetert wozoen.

Daer fín dan in den booefy Faxe 1595 / ban wegen den Generale staten ier Bereenichoe J Jedertanden ende fin dexellentie feuen ichepen tae-gemaecht om te 3 enlen dooz de Waygats of Siraet $\operatorname{van} \mathrm{Naffouwen,} \mathrm{na} \mathrm{de} \mathrm{Coningljrijetken}$ wan Cathay ende China, twee tot $31 m=$ fterdam/twee in 2 erlant/twee tot dine= hupfen/ende een tot lifatterdam/de feffe met aflerleu foopmanichap ende gijeit geladen/ met Commifen daer bp om de hoopmanichap te Déjuen / ende het $\mathfrak{k}=$ bende een Gacljt / die bebel lyadoe als de andere Grijepen om de Caep de Tabin (alielyouden zijude booz den untterften

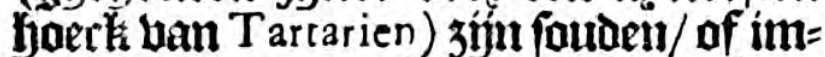
mets po verre/ dat ju zundelick mochten aengaen/ende geen quaet of berlet meer

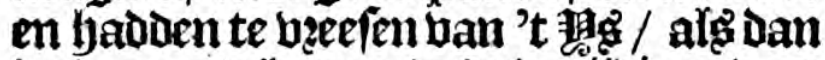
wederom te keeren/ende de tijoinge daer ban te bengen. Bp't groot Srbip luan ¿mfterdam wag soljipper endestuur= man de boonuemde Willem Barentíz, tot fommiş hebbende Jacob vanHeemskerck, de felbe die ontrent twaelf jaren Daer na/namentlick Zunno 1607 . in dient neweloigen sclyeepsi-ftrijt binmen de Baey enue onder' $t$ geffhut des $\mathbb{C}$ afteels bai Gribralter, den gemennen Dadex= lande ende lyem felben een onfterfíctiten naem beeft berwogen. Alset Dit felue Soljip boer bock Gerrit de Veer, unt

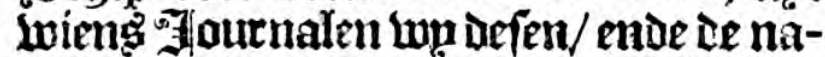
bolande derden tarbt katelick lyeluben getrodten.

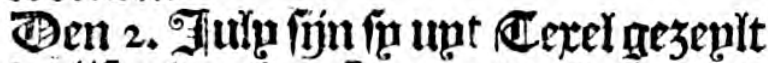
in't rij́f ban der sonne / ende taregen den 14. dito't lant ban Noorwegen in't geficht. Ten 22, na de middagly 3. 3. w. Son fagen fucen groote Malbifity recljt boo? de Loegl leggen fapen / die doo? 't gredunich ban't aen-zeplende Schíp en: De 't geroep ban't bolck warker wert/en alfo ont fwommen is / ander badoen in hem op 't líf moeten zeplen. T'Den 4. Flu=

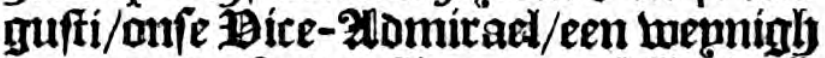
boos unt wefende/ftiet op een Hlip/Dach Don? man weder geraelaten daer licftes: lijekten ban / lup fultr yernemende beb: ben't voosta gewent. Den 6. dito/ qua= men wh en den Dice-3amirael tegenta den anderen te ftaten / maeckende mal= fanderen feer reddelong. Jon 7. Dito/ ontmoetede haer een Enchbupfer fethip hamenue unt de witte zee / Den 14 . Dita mett de booghte deg gools bebonden 70. graden 47. mínuten/ ende den i 8, ja=

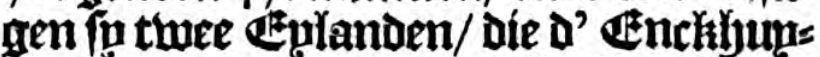
kers Den naem gaben van fín Excellentie ende ban fín beaeder Graef Hendrick, fas gen oork wefter son ten feluen dage de W aygats p.n.o.ban baex legten omtrent 5. múlen / wan de 70. graden tot aen de Waygats zenloen fu meeft al dooz getoga=

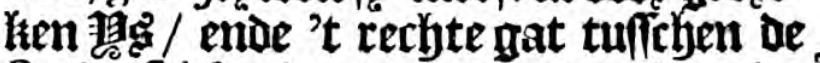
Beelt of Afgoden-hoek ende der Samoieden lant wag Daer fo bol af / Dat het niet wel mogelícli wasi te pafferen / daetam fo op de reede aelopen iñ in de 25 aep die fó naemben de T raenbaey, am dat fu daer beel fraen bonde. Tít ígen goede baey

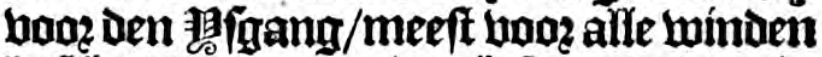
befefut/ende men mach fo berre daer in zenlen alfo men wil / 5 .4. ende 3. vadem goede fteek-gront/Dach bu den ooft-wat is't diep/te water.

Den 21. 20lugufti/tracken fu ap't lant ban de Waygats met 54. perfoonen / om te bernemen nae de gelegenthent aldar. Omtrent 2. mílen te landẹwaert inkas= mende/hebben fug gebonden berfelyenden fleden met Befle-werck / firaen / ende diergelijcke waer/oork boetftappen bam menfinen ende wheeden / daer doo? fu bermoesen datter menfeben amtrent marfen moneil / oft immers ltamen hanteren. Dit marbtmen te meer befpa= ren aen de menigte ban beeloen/die men aldaer op Den Beelc-hoeck (alfo ban lyaer 


\section{Om't Noorden nae China.}

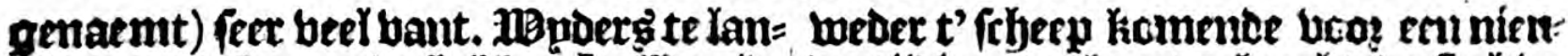

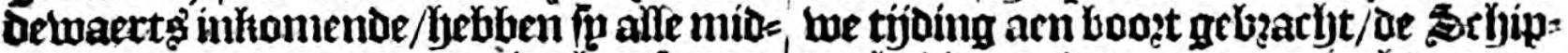

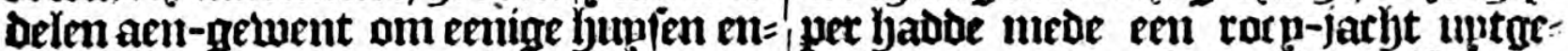
De menftyen te bunden / waer boot fip on= fonden oun te fien oft be citartariftye $3 e e$ Derbefen mochten wozben ban de gele= open was / maer fu mochten in be zee

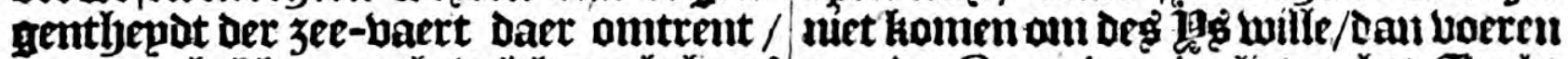

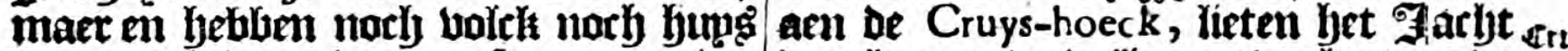
fommen binden / daetom fo tot naerder daer Ieggen / ende liegen ober lant na de bort

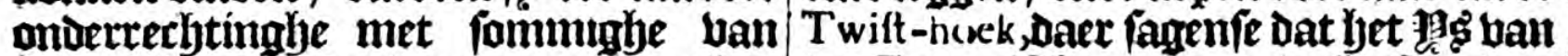

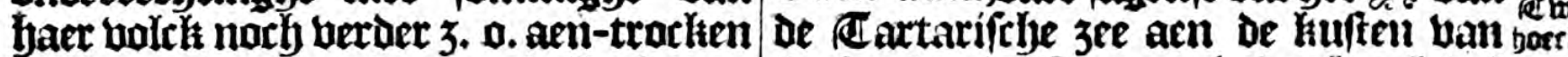

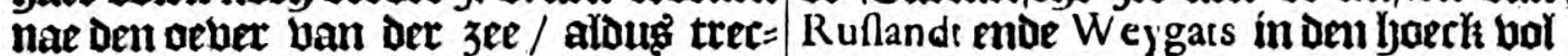

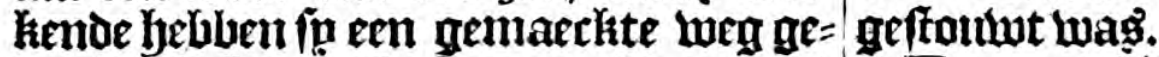

bonden in lyet mog of morag / ontrent Dell 23. Dito lyeblom fo ecn Lodgie ter balber knpe diep / want alfo diep tres wan $\mathrm{P}$ it zore gebonden/Die ban baft te fa= dende bonden fu harden gront / op 't al= men genarpt wag / ente badoe ncogte lecondiepfte loag het ouer de fehoenen waert op gelweeft om aldaer ecnighe

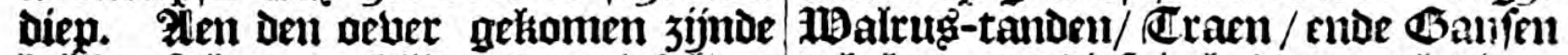
bebluen fo lyaer berblijt / obermitg ljaer te bekomen / Diefe in hadoen tot ladirge

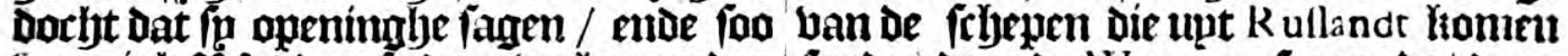

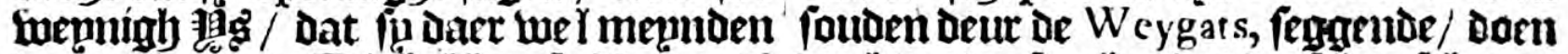

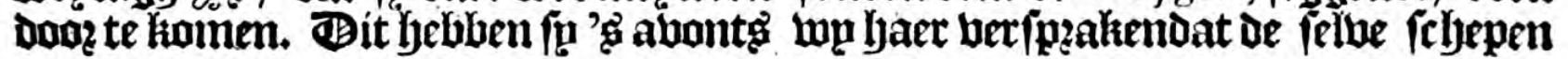
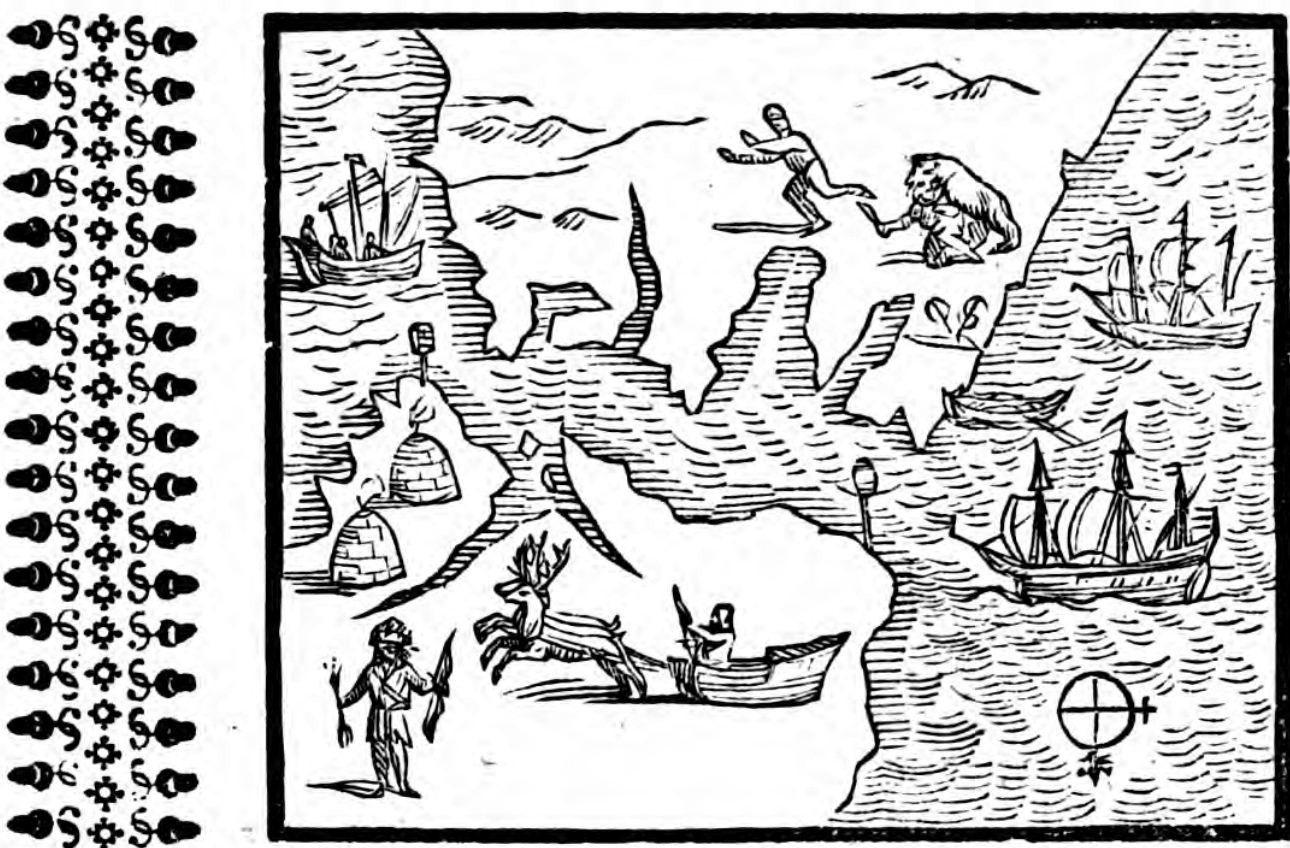

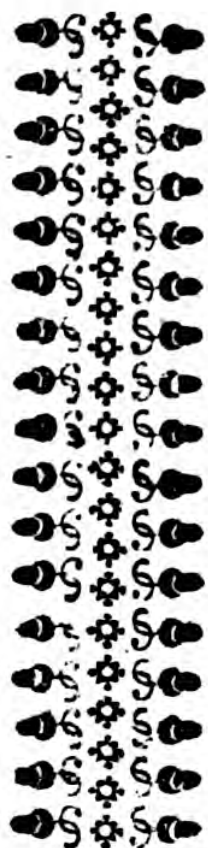

fouden Kamen om te zeplen in de Jarta- $3 \mathfrak{E e} /$ die fa noemben Marmare. Den

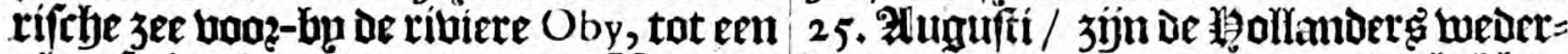
plaetie in Tartarien genaemt Ugolita, om mae de Lougie gegaen ende ljebluen

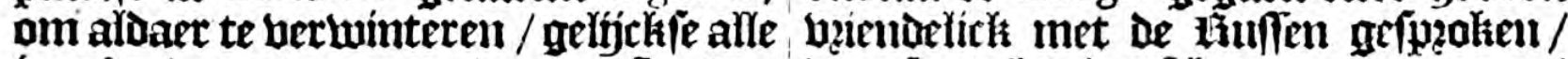

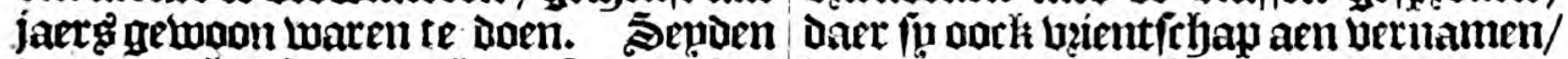
boozder / het foude nocly 9. of ro. Lueken want gaben lyaer fract in't aetilionien aenlopen / eer' 't gat foude tae viefen / aclit bette gaúen / die fu beel ín't rum maer toe wefende/dat men dan ober 1 gis mocljt loopen tot in Tartarien, ober be ban haer Lodgie badoen leggen. De bu= fe ber forbten/ fị willoen ren of twe met 


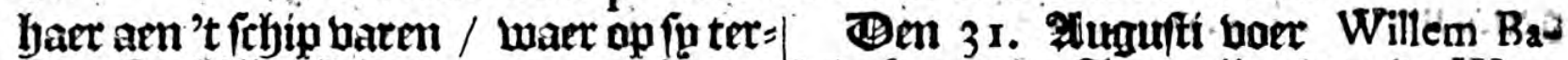

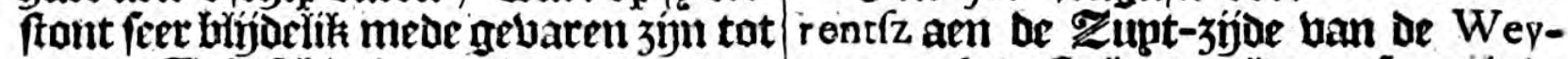
7. tae. Fn't felyip fiomende bertwonoer= gats aen't bafte lant / allwaer jue enighe uen fon lyaer feer bande grootlyent all gae = witde menichen / genaemt Samojeden

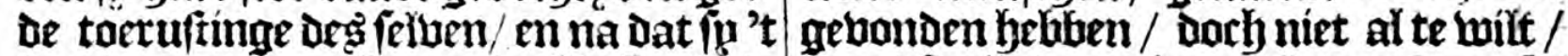
bau booren tot acljteren wel be/ien bad $=$ want fug jijnde 20 . in getale/met be onfé den/hebben de onfe lyaer t'eten boos je= die negen waeten/ ppaek gebouden beb:

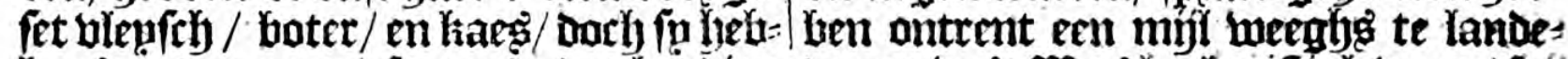

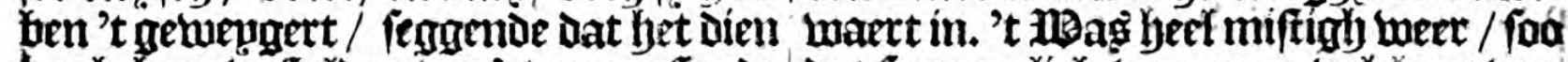
bagth bare baftetoag wag / maer fiende dat p monelick ban geen bołck en vers ten laeften pelielfyaring/ lyebben daer af = moeden / ende de onfe fagenife in thee Ie te famen ban gegeten / met ljooft met boopen bíf ende bijf te famen gepar= ftaert met al/ban boven af bijtende. BE $=$ tuurt / ende waren feer dicht bu haet ert

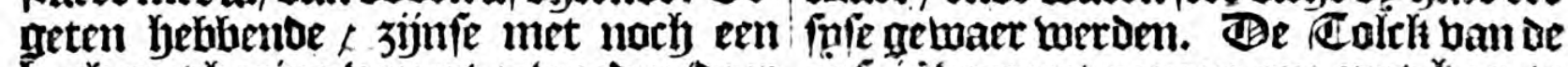
back met lyaring bereert yeluogden/saer onfe is boos unt gegaen om met haer te

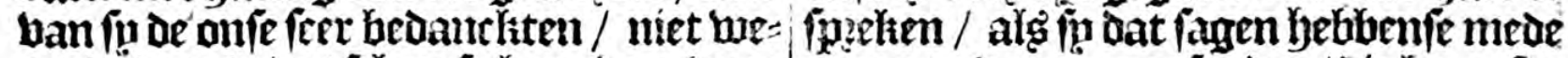

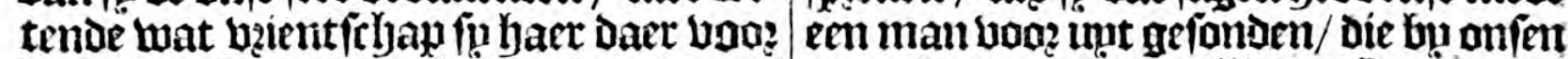

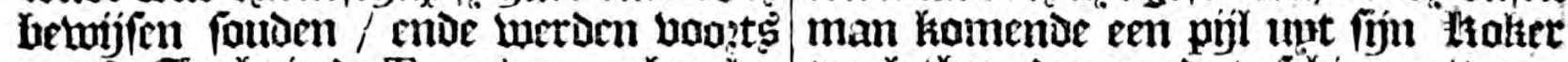

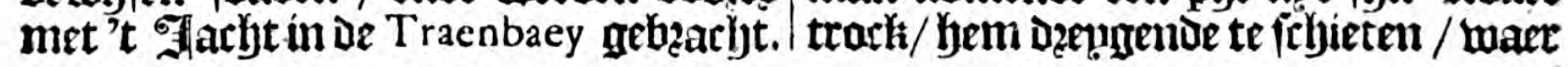
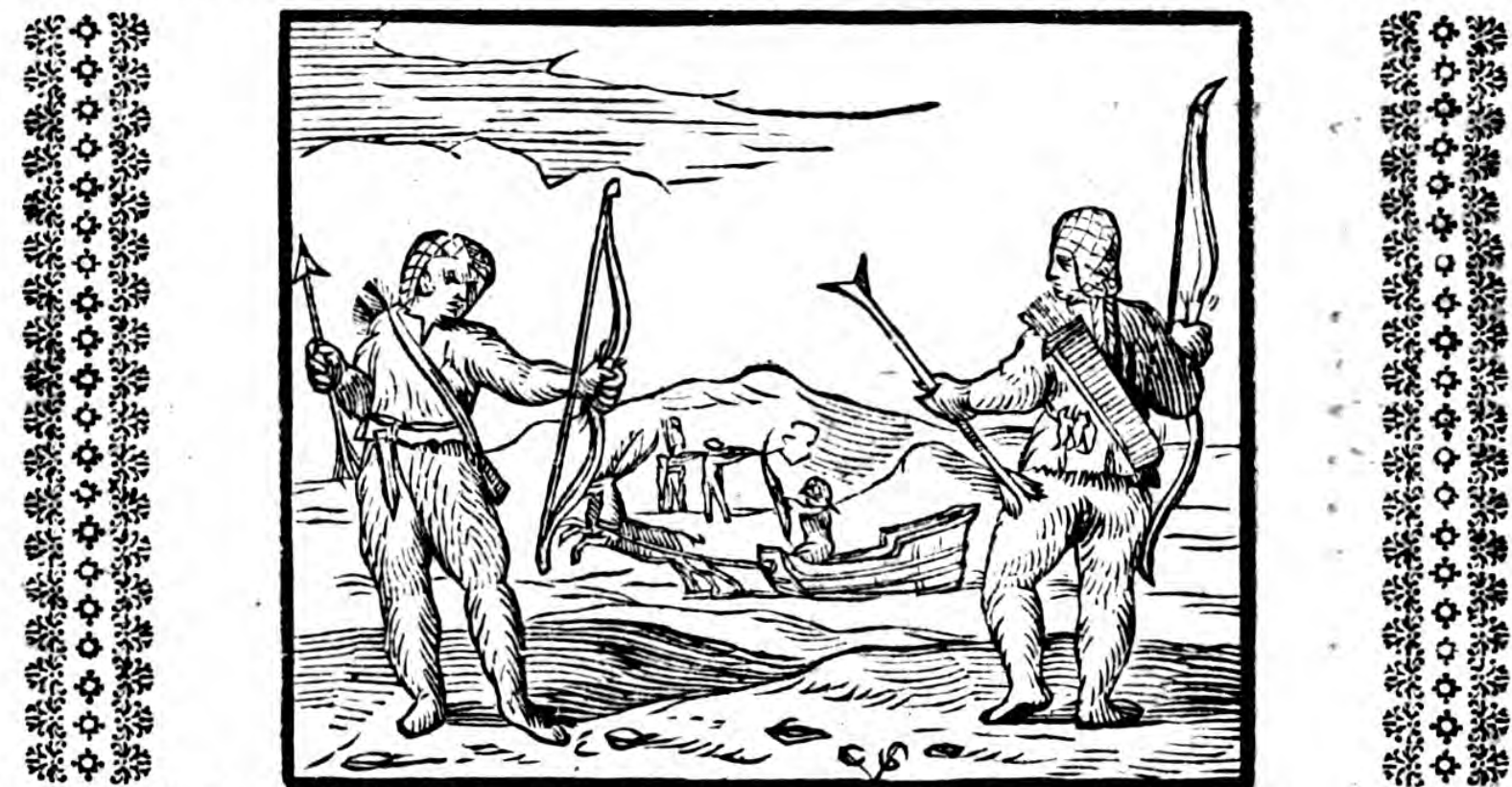

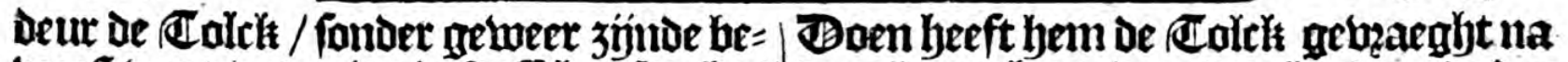
beeft wert/ende riep in linfelyet ppatte/ fifiet niet / wp zín beiendon / o' anoer dit boorende / heeft fín pijl ende boogh ter aerde getwoupen / Daer mede te ken= nen gebende dat hy wel hegrerde met onfe folck pratte te bouden / de melelte andermael iegrende / woj zijn bienden/ beft beeft de wilde geantwonet / fijt dan wel= wel wiften / obermit een ban baer daet

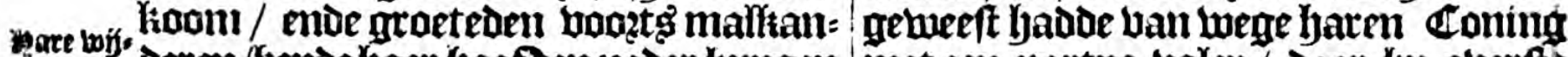
ie Dan Deren/Gepde haer hoof den neder bungen met een partpe bolex / Daer bu duerfte sroeten. De ter aerden/ nae der fufen maniere. ban getweeft was. 
Om't Noorden nae China.

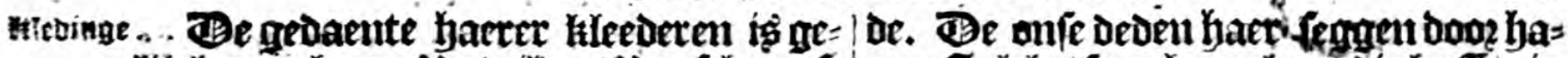

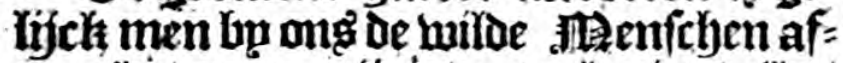
maelt / maex jijn ebenwel niet wilt / want gebaucken goet verftant. Sp zijn geftleet met bellen bau Iflyeeden uan oen boof de tot de boeten / untgefoniert de boomaenfte / Die't booft bedecken met eenigb gecaleut II aeken gebordert met pelterpe / De antere dzagben alle mut:

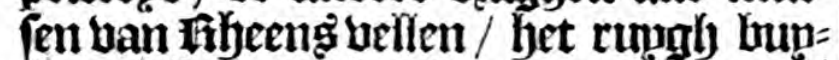
ten/Diclyt aen't hooft gebargt/ende luel paffende. En Dzagen launglyage in een blecht geblochten lyangenoc binten haer Kileederen op den ruguge / zijn meeft fiogt

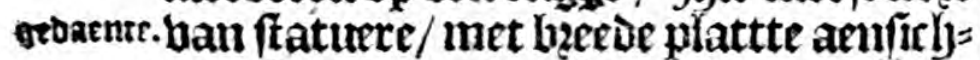
ten / Glicpne ougen / Yoote beenen / laaec fuijen fiaen untwaertes / ende fin feer rafel in 't loopen entor fyingen. Ben beemoen fletien hetroumen fu hepnity/ Dat bleech Dart aen / Dat als be onfe/ (Die lyaet nochtang affe eere ende byient= fillap betweien baiden/ den eer/ten Sep= tembigig andermael aen. lant quamen / ende een boough ball haex enfeliten om die te befien / Dat fu't weygerden / ende eqn tepelten deben niet daer toe te willen betfaen. De gente tie fu haeten $\mathbb{C a}=$ nimgly noemoen ladoe feljilt-waclyt unt= faende/lettende op't gene datter omine gingh / ende gekocht of t betzacht wert. $\mathbb{U} \mathbb{E}^{2}$ ban de onfe genaefite hem luat na= Dex/ende betwers bem beient felyap na de bujfe by baet gebeupelieliek/ lyem gevenDe een bifruyt't welck by inet groater eerbiedinge aen-nam / ende terfitont op at / maer in't eten / als pock boo ende naex/nam lyp feljerp regard op al Datter omgingh. (Gaer feden ftomien altijt veer abroen. Digh met en oft twee fibeedrit ingefpan: nen / vie alfoorafth homen loopen met een mam oft twee daer op/ Dat ljet geen ban onfe paerden daer bu herden mogê. Uten van de onfe frljont imet een Jlauf= ket t' Zee-waert in / Daer van fu fo feer ber/ctyeichten/ dat fe liepen ende pigangen als unt innige menfchen / Doch befadig oen fu baer weoer / fiende dat bet unt geen quaetwillicheut of arclyeut gef bie $=$ placte te doen fien wat kracht men daet mede doen konde / fo lieeft ren ban de onfe een platie fieen / ban een lyalbe palm groat / een ftuck twecg!j wan bem op een bergh gefteit/ mue fp wel fiento bat fulcr uet te bedugdent hadde / jijn in 't ronde gam fiaen mel 50 . oft 60 . il getale/ fo wat af: wijekende. Doen heeft ly die de rus hadde na defteen gefelyoten/ende die foo geraerht / Dat lyy in ftutien vicl / lyicr auer waren fu norh meer bermondert alg te borm. - Daer nae liamen huy ong af-felyent ball malfianderen met moo: ter eerbiesingbe aen lueder zjobe/ enve in't Tarfjt zijnur fichten lun andormael

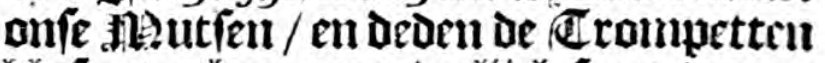
blafen tot lyaer eere / gclijek fị inede nae hate Lijfe ong alle ecrbieuninge on bient= fichap betwejen / gaende boost nac baer fleden.

foae dat fu ban ong gefelyepuen ende foo wat te landewaers ingerepft waren/ iffer een ban haer op frant komen rí $=$ Den / om te haleneen beelot unt den rank wen gefineden / Dat de onfe bau' 't frant mede genomen badoen/ende in 't $\mathbf{F}$ acht gelept. Zlla lyp nu in het Facht was g $\varepsilon=$ fomen/beeft lyp dit beelt glyefien/ende de onfe een teycken gegeluen / fu hadoen groot quact daer aen gedaen dat fu dat jeelt genomen badoen/De onfe dit mer= hende ljebben bem 't beelt wederam glye= geben/ende bu fteldet daer op een bergh dicht aen den ocber / fant daer een flede om/ende liet lyet alfoo baelen. IRae't aee= ne de onfe beinercken lianden/pa bieloen= fe fulcke beelorn boo? baer Soden/Lwant op den ljaecli bande Waygats, die de anje de Beelchoeck naemben / banden fu ette $=$ afgod licke hontordon fulche gefineden beelden/ beel roum / boben wat ront / ende in't medoen een ljetwerlken gelaten in plaets bau een neus / bouen de neufe thee ine: den in plaet fé ban ooglyen/ende onder de neuse een mede in plaets van de mont. 
32

Tweede Schip

Sp bomben oort boos de beeloen / beel afficle ende beenen ball tijeeden / Dare upt te qiffen igid de inwonders aldaer bare of exhanden gedaen lyebben.

Den berden Septembiga bebluen py ' moareng 3 eul gemaeck omtrent twee uxen boo?'t rijen ban der son/ende quanen metter Sommen opgangh am= trent een mijl bu ooften de T' wilt hoeck, zenlende op ten nooziden / tot de zimber Son / amtrent feg mílen/ inaer daen maeften fu't dooz de beellyept deg de d'ougbeftadighent deg wints" / als mede dooe de dicke mift/Dickmaels wen= ben/ ende berbieten epnoelick op de anftS'a'en Esplant.

Boorots zijoe ban het Staten Eylant, omtrent een murket fifjoot ban't lant. Op Dit $\mathbb{E} p=$ lant gingen fu altemet die daer feer beel waren; tembigs deg mozgengs zijuber eenige 23oot's-gefellen aen't valte lant gegaen om ghefteenten te forthen / zijnde een 䄱e manten. Eylant mede feer beele waren. In't foet =

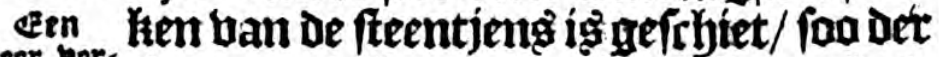
niert troce twee maet's bu malkanderen lagen/Dat=

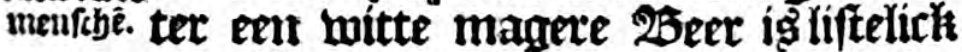
aengeftomen/ende greep/ D' eell ban de= fe twee in de nels / hp niet huetende wat het wag / riep / wie grípt mu daer bam achteren! sijn maet/ die bu hem in de I upl lach / fichte fijn lyooft op om te fien wie't huefen mocht / ende fiende dat lyet cen $23 e e r$ was;/ riep och mlaet/het ig een $2 \mathfrak{B e e r} /$ ftaende mits dien wel wacker ou / ende loopende al fijn beft wecls. TBe 2ier beet den eenen terftondt $t$ ' jooft in fucken / ende foogh bet bloet daer unt. 't inder boleli dat mede aen lant quam/ fiep flucr der twaert wel 20. ftereft/ om de mante berloften/oft ten minften ben 25eer lyet doode ficljaem 't ontjagen/ inaer ałs pin haer toergi endespiefen ge: belt hebbende na oen 2 Beer toe quamen/ die noch ftont ende at ban lyet doode lic= haem / foo igs bp feer weet ende onbe= fclyzoom tot baex in-gelopen/ende beeft norty een man unt baex wectygerulat en=

\section{Willem Barentfz.}

De berfelyeut / waer Doos de andere al te famen de blucht genomen bebluen. THe ban't schip en't Iacht fiende dat haex bolek geblucht quam nae den aeber/fijn metaller baeft in de feljupten gevallen / ende ha den oever geroeyt om haer volfe t'onfretten. Fen lant fomende hebben fip dat deerłich felyoufpel baul lyaer boicft gefien/ende praken derlyalven malftan= deren een moet aen/ dat fe gelijeker bant teugn den 2 beer fouden aentrer fen met Hoersi Coetelafien ende balbe ilancien/ fonder Dat petmant wíelien foube / Dach dit en fionoen fut alle foo nirt berftaen / caut fommige fepden / onfe maetş zijn boch al doodt / inp fullen oen $25 e e r$ wel kríglien al en ftellen wu ong in fo open= baren perijeket niet / mochten wp onfe

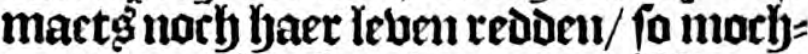
ten wu ong baeften / marx nuis't met reen baeft te doen / Dam wn moeten bem gewis nemen / ende dat tot onfe meefte berféliertljept/ want bu beblien met eetr weert fel ende berflindende beeft te fef af: fen. Joen zínder dzie ban de maet tat wat bet boost aen-getreden / De $25 e e r$ even= wel boot-barmde fijn aes te berfinden/ ende op alle hare menighte / boewel fo wel 30. fterch waren/ niet achtende. - Defe dxie Lwaten Cornelis Jacnbfz. feljip= per ban Willem Barent\{z Willem Gyjen Stuurman ban bet Flacht / ende Hans van Uffelen, frtyejuber ban Willem $\mathrm{B}_{2}$ -

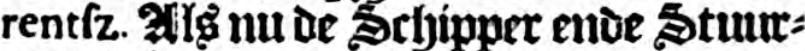
man deiemael gefeboten ende niet unt= gerecht lyadoen/ iq de feljeyjuer noch wat bet booz unt getrésen / ende den 2 beer biumen feboot tiende / ljeeft met zijn Hoer daer op aengelept/ende lyem doo?'t booft bn de oogen in-getteft / maer de Seer bieef noch al de man bp de neck youdende / ende beurde ziju booft nocl op met man met al / Docly bergonft wat te fupfebollen/Daer ober bebben de felyeí= ber ende een scljot/man lyem op de lyupt geflagen met haere Coetelaffen/fo datie in ftucken fwongen / fonder dat by fín aeg noch barlaten wiloe / in't epnie is Wil- 
Willen Gijfen toegreloopen / ende beeft daegbs boeren fi aen d' anber zjjoe ban be sber ben $23 e e r$ met fijn Boer ober be fmut de Weygat s nae't baftetant / om 't Cae wozt ges geftaglyen dat bp boen eerft ter aerden nael te diepen/ ende boeren beel in de boot. biel/ enoe W illen pyzangly hem op' $t$ lijf/ ende fuet bem de ftrot af. Baer nae bebhen fy de doode tichamen den 7 . Sep= tembiga op lyet Staten Eylant begraben / den 2 Ber gevilt / ende 't bet $t$ ' Dani gebzacht.

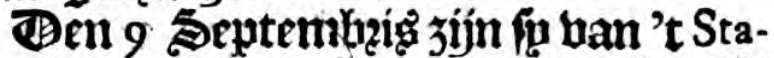
ten Eylant t' zenl gljegaen/ maer bet 19 quam po beel enue getweloigly ím / bat py liet bcoz en morljten / ende 'gabontts moeften weder keeren daer ig 'soz= geng̉ waren af-gebaren / zentende den Tomirael ende't Tlacht ban lotterdam op de klippen/ Dorth fonder groote febade Daer afrakentor. Jenl I 1 , Dito zeploen fo weder ím de. Tartarifebe zee / maer ge: raeckten andertwerf in beel fu mederom nade Weygats zeploen/ende fetteden 't by de Cruys hoeck. Den 14. begoft bet luere te beteren / de lwint liep ten n. m. ende de froom quam ftijf bocht aryter lyet tylandt met de fteert, daer ip een klepn bumfken ban bout bon: den / ende een groote afwateringlie. 's nazgeng wonden fa 't 3incker aen boot ende de fteng om boond/ meenende andermael te berfoecken om hate rep: fe te berbozderen/maer d' Fomirael ban ander meenínge jijnde ia bijijben leggen.

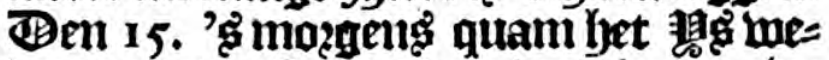
derom lyet ooft-eunt vall de Weygats in. Dejíbell / fo dat fi fract geomongen wa= ren lyare sinckers te lichten / ende fín

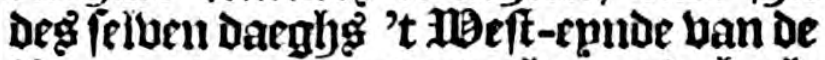
Weygats meder untgezenlt met de beele blote naer bups toe. Den 30. Dito qua men fo in't epland $W$ ardthuys, daex wp eloerģ beeder van felyetjoen fullen / ende lagen daer tot den 10. Betobeig/op weleken dagl fu wederom t' zepl gin= gen/ende fín den 18. Anobemberig daer aen in de In aes gettomen/na dat în unt= unt de J artarifthe 3 ee loopen/ Deg feltill gelweeft hadoen 4 maenden en 16 dagen.

Kort Verhael van de derde, ende Alder-wonderbaerlijck@te, feltfaemfte, jae te vooren noyt diergelijcke gehoorde Zeylagie, by den voornoemden Willem Barentfz.achterNoorwegen, Mofcovien ende Tartarien om, naer Cathay ende China gedaen, uyt de name ende van wegen des Eerbaren Raedts der vermaerde Coop-Stadt Amfterdam, in den jare 1596.

$$
\text { Mede getrockinuyt bet Fournael des vosrnoemden Gerrit de Veer. }
$$

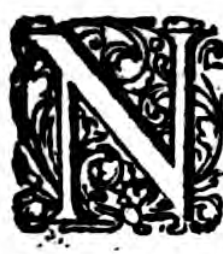

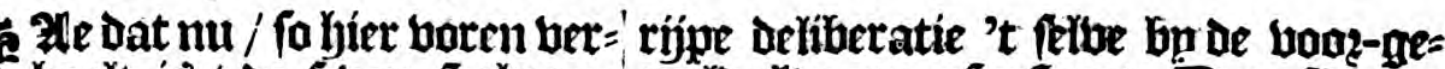

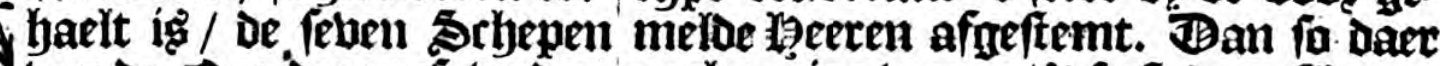
ban de z

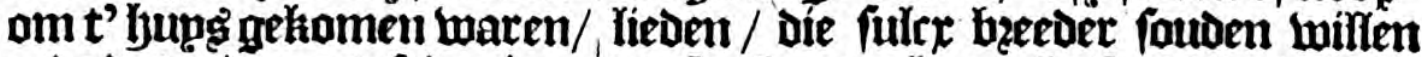
ende dat niet met fadanigen berfuecken tot haren kofte / dien wilden

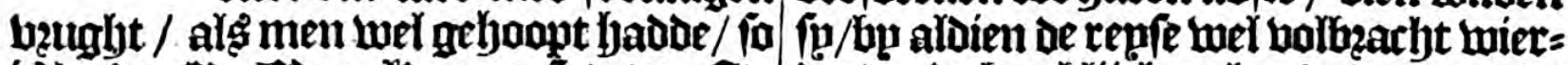

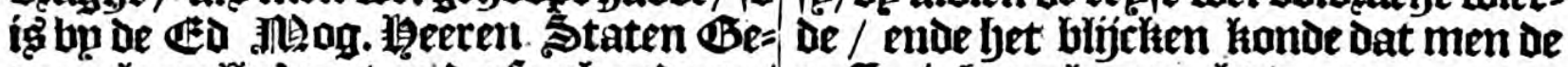
nerael op afteg goet onder/aeek gedaen/ paflagie bezenten morbt/geetne een goes ende de fake in beraet genomer / of men be bereeringe ban 's lantş wegen doen/

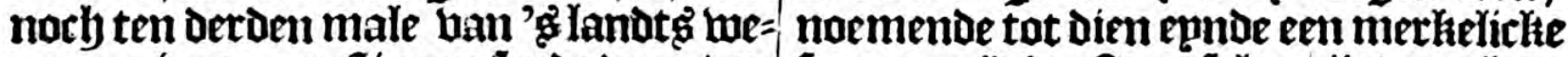

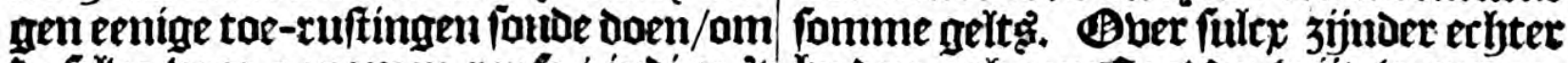
De felbe booz-genomen regefe / indien' 't hy den eerbarenlfaet der wijt-bermaer=

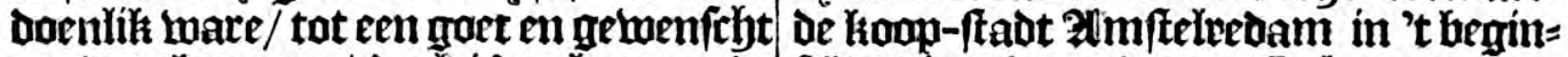

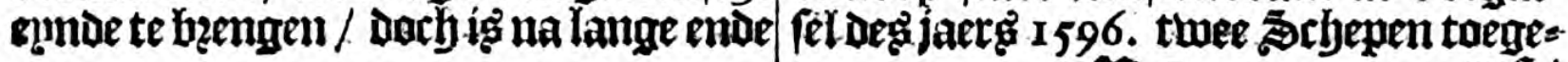


14. Derde Schip-vaert van Willem Barentfz.

kuft/ Baet wan het feyeepf-bolch op twees Derlen conditie aengenomen 3 ijn gewos=

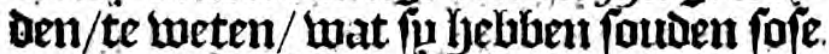
onberted Jter fafte wederam quamen/:n=

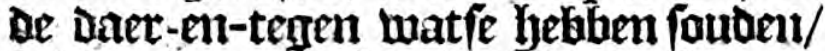
Indoien fo de repfe ballbartyten / yaer in fuften gevalle/belouende/om lyaet maes digh te maften / een merkelijełte goede be loninge/ ende fou beel angethoutoe per= foonen daer tae nemende als fu eenigly $=$ fing machiten/om te min booz ben treek tot wijf ende hinderen in 't werk te vert= fagen/of ban De repfe afgetogen te twoz= Den. Op defe conditie zîn de twee Brbe=

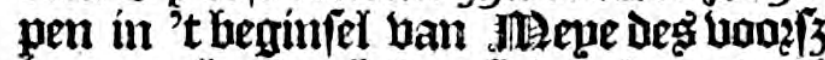
jaren zepl-rede glyetweeft / ap't cen wage alş Erhipper ende Clommigh ban de Hoopmanictyappen de boomaemde Iacob van Heemskerck Heyriderickfz.ende Willem Barentfz. ald Bpuer- 5 tuur= man/op't ander Ian Cornelifz. Rijp, ałå fehipuer en Commies van de Koopman= felyaupen bin Den Kaop-Ytugden daer íme

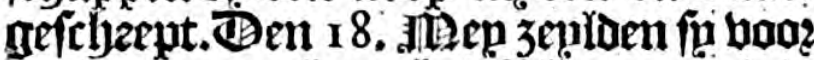
de tweede mael tugt lyet datie / ende den 22. Fregljen fu de Sethot felye deplanden

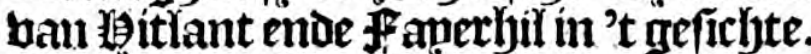
Dinn 30.warē fó op de hoogte bau 69.gr. 24. Min. en badoen den eerften \$ump treen naclyt.

Den 2.Dito / ontrent \%. \%. (B. Son naonor inch te: fagen fu een feer Luonderlijit ligemel-tep=

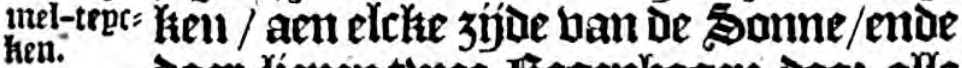

daer liepen twee litegenbogen doos afle Die be sommen benen / ende daer-ell-bo= ben noch thee lategenbogen/ d' eene hijt rontamme de Sannen/De andere divars boo? fyet groote randt / Dit groute rondt ftont de anderfte kant berljeben bouen Den Sorizon 28. oraden / deg midoarga

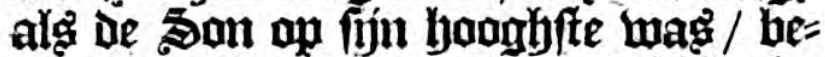
bantmen boo? 't meten deg alfrola= hiumg / dat fu waxen op de hooghte han oserbil = 7r. graden.

tufnthen 't Bchip van Ian Cornelifz. bielt de

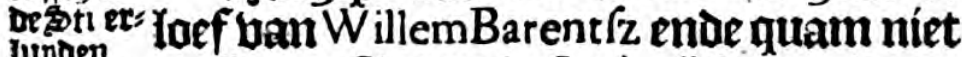
ban be tot lyem af/maet defe gingh den anderen

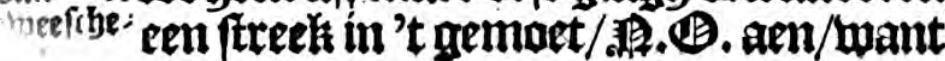

bem Docht lyn mag al te wefteliget $/$ fo't ook namaeldibleeck. ats fo mu? abont bp malkanderen quamen fepde bem Willem Barent\{z. Dat ip nach bet oofte= lích moeften aengaen / om dat íte ver= re lweft-waert luaren / maer Ian Cornelifz. Stuerman gaf tot antwoogat / Dat fin niet en begeeroen in den $\$$ Intham ban de Weygars te hefen. Haer rourg bag

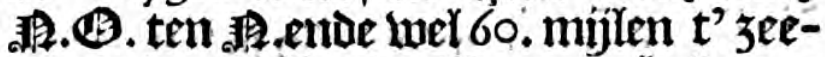
waett ban't il and / daetom lyad men Daen mennde Willem, beet eer beljoost

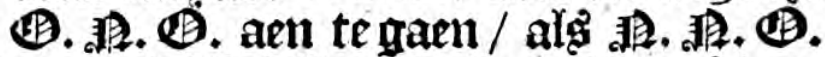
obermits fu fulfen ftucli om de tweft wa= ren / ja men lyadoe beel eer beljoost onft aen te aaen/ten minften eni Deel mijlen/ tot dat mell weder te recht gebearht hadde dat doos de quade winden aerlo: ren luas. Doch en wilde dieg niet tegen= ftaende lan Cornelifz. anderte niet dan

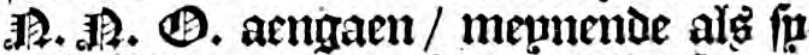
onfelijefter aenuaen fouden / Dat ju dan in de Weygats fauden Kamen / Dies ljem Willem een ftreeck te gemaet ging/ende 3enlden a. O. ten 3 . aen / daer pu an= Dert 3 . 19. ja noch ooftelícher fouden aenuegaen bebluen.

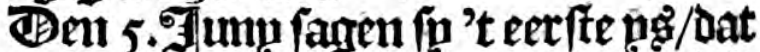
yaer feet wonder gat/ende meynden in't eerfte dat het witte Blwanen waxen / fo dat een ban lyaer wandelende on 't ber: defe onberfieng begon te raepen met lup= der ftemme/Datter buitte sluane fwom= men / die onder waxen fultex hoorende fpzongen fitur op / ende fagen Dat lyet ys was' / 't welch ban de mooten hoop af quam Dżjyen/en mitge dien yet tegen den abont wag / wel Slwanen weleeck. Deg midoernachte 3eplen fú daer boo? / ende onen was de son wel ontrent een graed

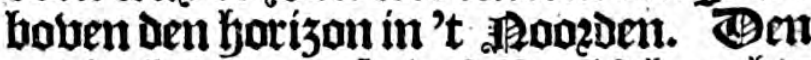

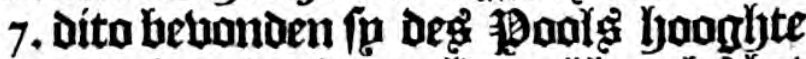
74. graden / ende zeploen al langly bet ngi henen/algs of fu tuffeljen twee landen qezenlt hadoen: (1) water was fo graen als gras / ende fu bermoeden datfe bn Groenlant waren / fomende lancr om meer in noch dicker pq. Den 9, vonden 


\section{Om t Noorden na China.}

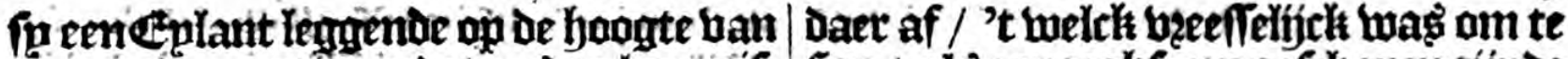

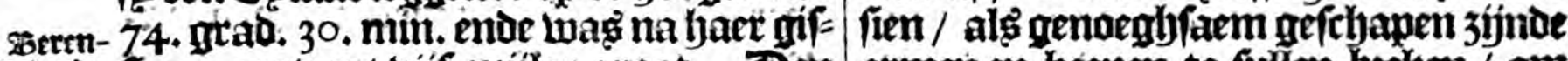
esplantr. imge outrent byf mílen moot. Ben atmen en beenen te fullen bęeften / om 1 r.aen lant barende boudon fp daer beef datter aen de boet van den berg fer beel Jilectuwen eperen / ende waten in proot períckel ban yaer lijf / want fing gingen een fteple fmer-bergh op / ende in 't af =

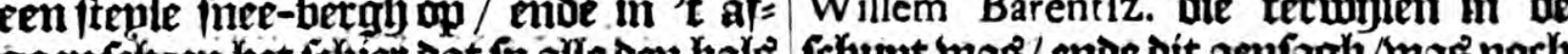

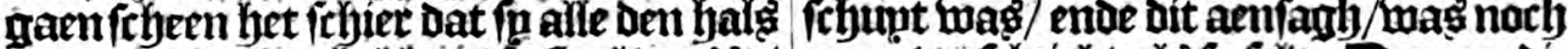

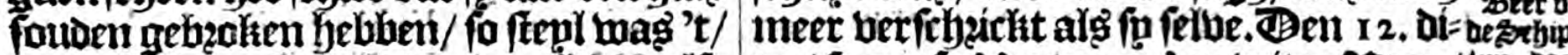

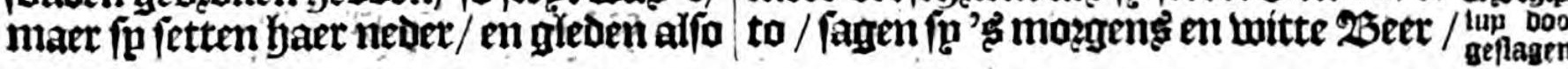
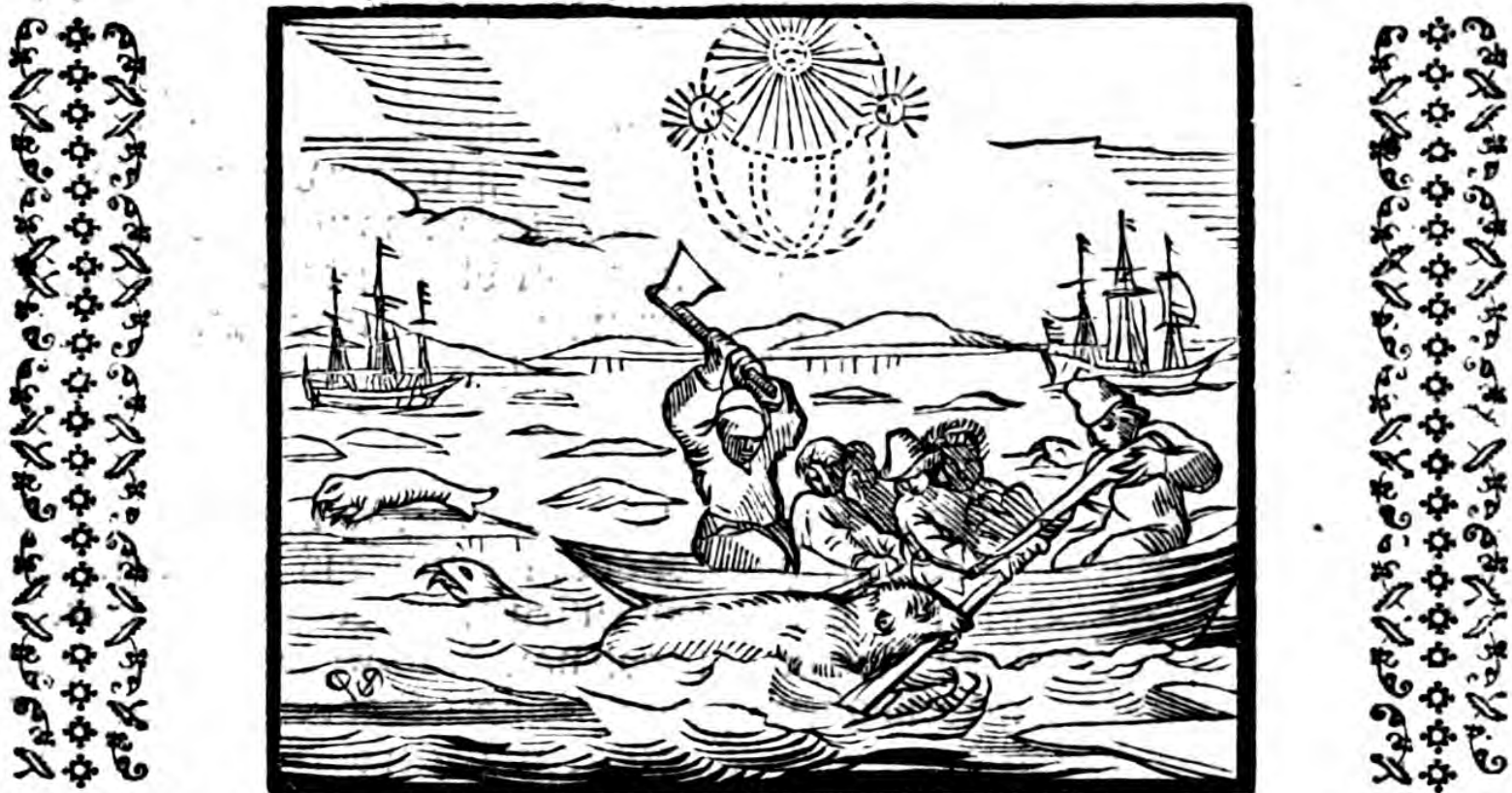

ende roenden met de felyunt naer hem groate meníghte ban Alectuwen op $\mathfrak{a}$ tae / meenende hem een fitick oun den

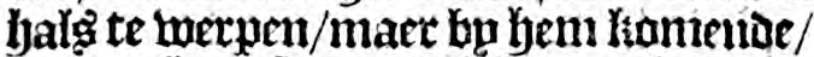
bonden bem fo gemeldialy / Dat fí 't niet en Doeften beftaen / hadoen derlyalben meer getweer ende bolck / ende lan Cor nelifz. maet quamen baet mede te $\mathfrak{y}$ ht $=$ pe / daer beftreden f́p't famen den 2 seer rel 4. glafenlanta/ mi bietwen tyem ten laet ften met een bijl 't lyooft in ftucketen/ fo dat de doot daer nae bolgove : Daer na hzachten fo hem in lan Cornelirz frhip /

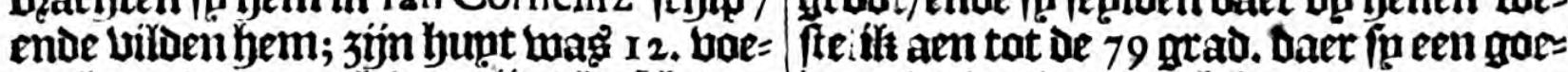
ten lang/aten nock bam 3 ín bleefth/ogr be reede bonden/ oocth tonden net naer =

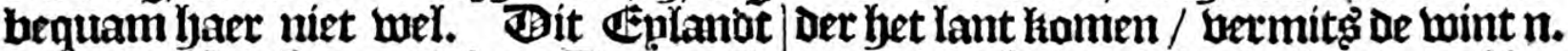

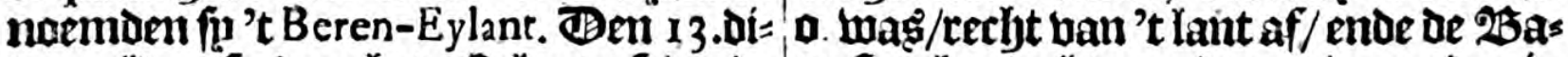

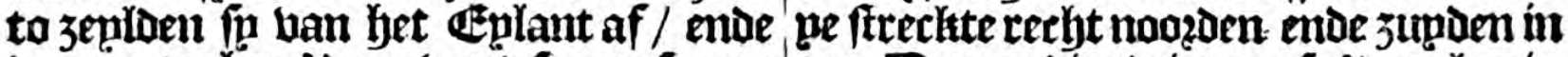

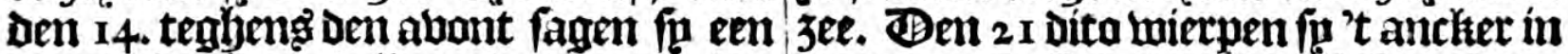

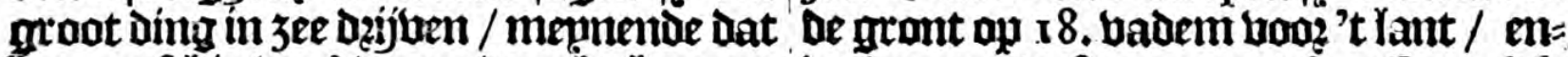
bet een fethip wags/maer daer bu komen= de boeten te famen met lan Cornelifiz

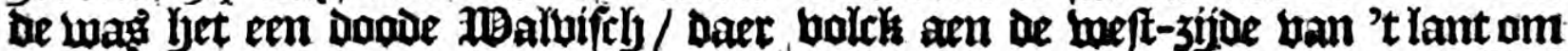
ballaft 
16

Roci een ballaft te balen / waer met în aen boost

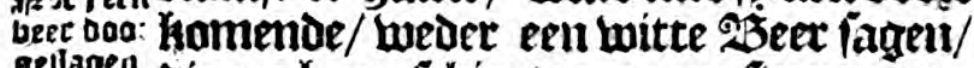
geflasea die na haer frlyip toe quam fluemmen. Hier boo ftonden fin baul fyaten arbent af / bielen gefamenter lyant te boot / en rorgden lyem na / maer lyp / twom / wel een mij' t' zeetwaert in. Sp bultroen lyem met 3 Fhupten aclyter aen/ende foegen meef al haer geveer op fijn bunt ín ftuc=

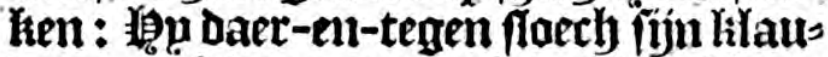

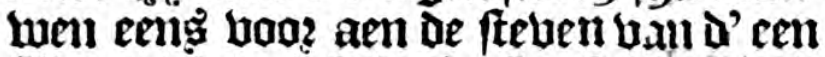

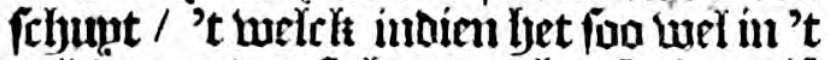
midoen gebueeft lyadoe/ hu faude mif́=

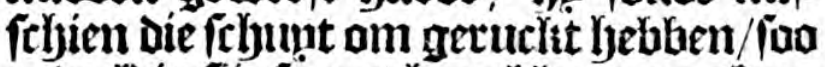
getweloíg fínfe met haer Ktaumen; \& Delick bebben de dzie febtuten met balk bem wermeeftert/ Laot geflagen/t' frbeep gebzactbt entor gevilt / fijn tjunt waga 13 . boet langly.

Daer nae raenden fu met lyaer ferlyunt wel een mifl te landetwaert ín/ Daer Luags een yoede haven/ 16. I 2. ende 10 badem diep/ende al boozt roepende betwonden fa

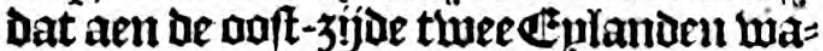
ren/die haer ooft in jee ftrechten. Ilen de weft-zijde wafier mede een getwetoighen Tuham / ende leecla wel meare en \& fant te wefen. Doen raenden fị booit

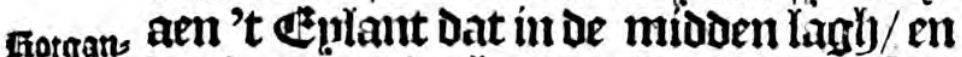
Een / endoe bonden Daer beel eueren baulfatgaufen/

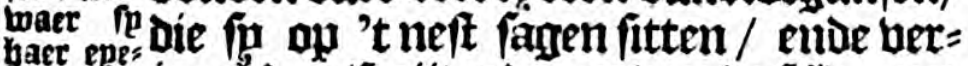
rent upt, jaeghoen//mijtende een van de fellue met byoberi. een fteen boot/ die fí Kaockten met on= trent 60. energi daer toe: (Bit waren op= rechte inotgaulen alfer in Wollant of Mieringen in grooter menigte alfe jaers ober-komen ende gebangen wazoen/ die men tot nocly toe niet gelueten en lyeeft waet fo haaer exeren leggen ende unt= bzaeden: Thaeram fommige fefhipperta haer niet ont fien bebiben te /efbijuen/bat= F́e in Scljotlant aen boomen waffen/en= de dat de betughten der felbe hoomen die

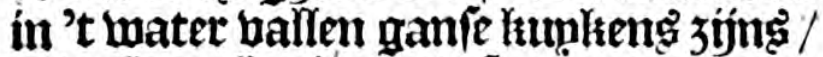
ende ftract begiment te f weinmen/maet die op't tiant ballen / berften in ftucken / moe komen te niet. Bit blijektat nu con= traxip/ ender is niet te betwonderen dat

\section{Willem Barent $\mathbf{z}$.}

niemant tot nach toe getweten beeft/

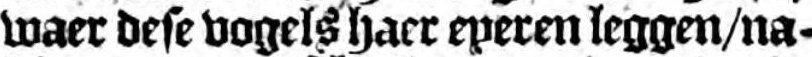
dien nopt meniche/ Dat men weret / ap de 80. graden getweeft beeft / ende dit laut nout bekent is getweeft / beel nin dat de hiotganfen daer op baer jonglyen bzae: den.

Hier ftaet nacli te bemercken / yoewel oit lant ('t welck 'wo aclyten $1520 e n=$ Vant te 3ín) op 80. waden ende nacly lyo aer íśleggende / datter ebentwel loober

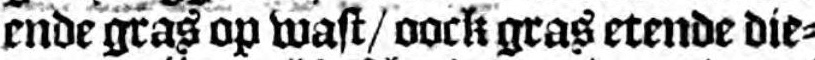
ren op zijn / als afljeeden ende andere / Daer nacljtmis ap Nova Sembla, Dat wet 4. maden fitif berder ban de aloogtpool lent / noch loober nocly grage en waft $/$ en= de niet dan blewfeb-etende dieten / als Berten ende Baffen ap zijn.

$$
\text { I N W ERP. }
$$

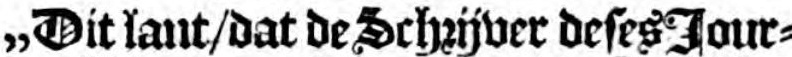

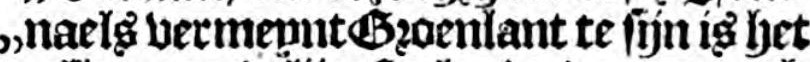
" allet-noozadijicxfte lanot dat tot noch ", toe out in kenniffe ig gefiomen/ende ") Iept tufficljen Groenlant (Dat onder de "froon ban Noorwegen behooat/) mibe , Nova Sembla (Dat den Mufcovijr toe(Kamt) regt tegen auer ten noazden ban "Finmarcken, ofte (foo 't in 't gemeen "gentoemt imert) Noorlant in Nourwe,gen, ftrectiende (nae fo bele als uan de " oule tot noch bebonden tås) ban de "76. tot boven de 80. mraeden ende bol= " yeng dien 60, Dount iche mijlen langhy

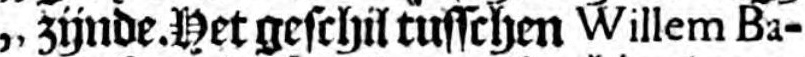
"rentfz. ende Ian Cornelifz, hier voren

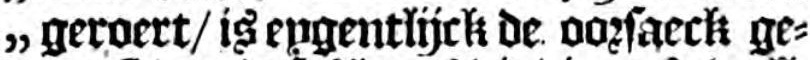

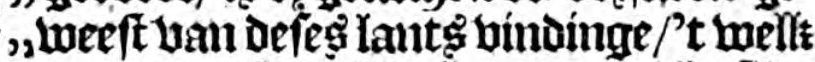
, aen de weft-fiuft th haer ontorcht fịns , de/Luederam Pthma r 608. vam Henrick

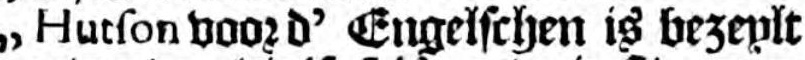

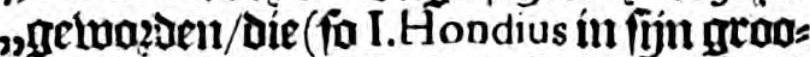

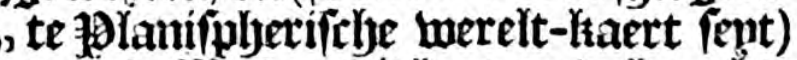

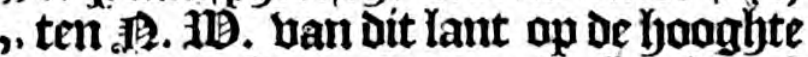
" ban 8 i en 82 .graden een bafte us - huft "rebonden herf. Sedert dien tijt thebben fu affe fomergeenige felyepen derwaert 1) Gefonden/oin IBalrufch-tanden/ mode

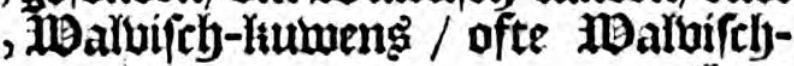


"been (fo men bat noemt)te fjalen/ende , legen / minter ofte hoster tijt Somor /

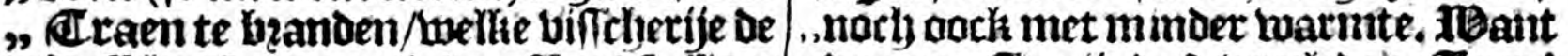

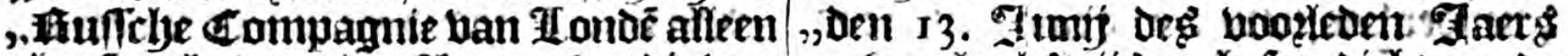

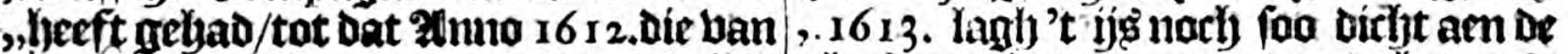

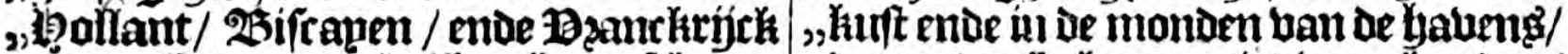

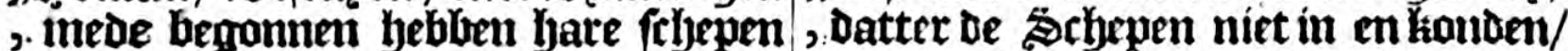

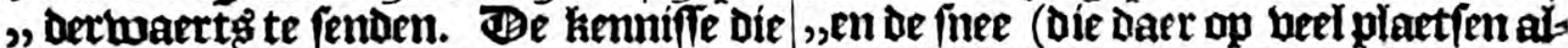

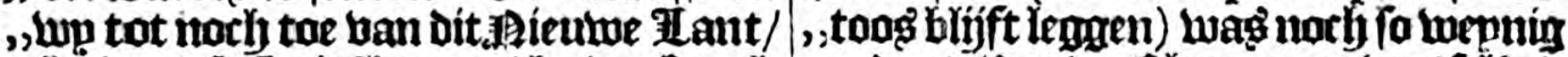

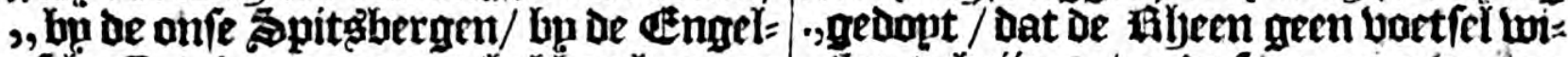

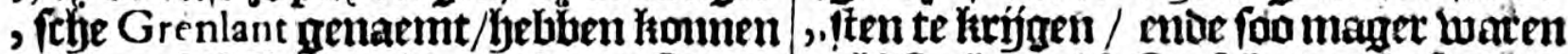

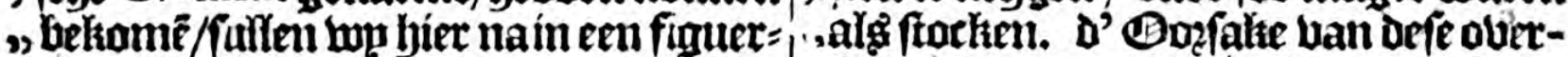

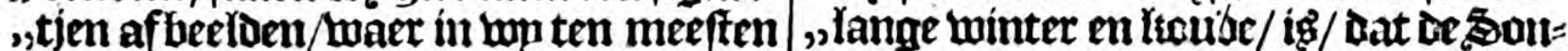
"Deele noch bolgen be teeckeninge wan , ne aldaer ten bougften maer en kilimt

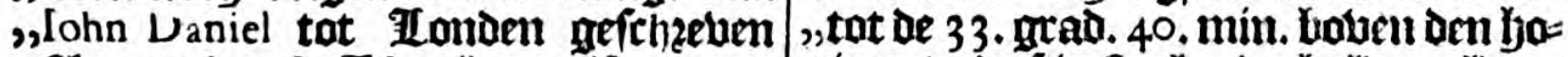

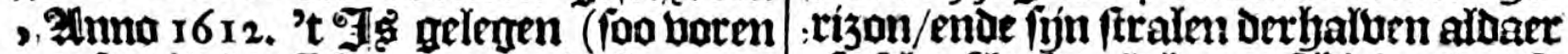

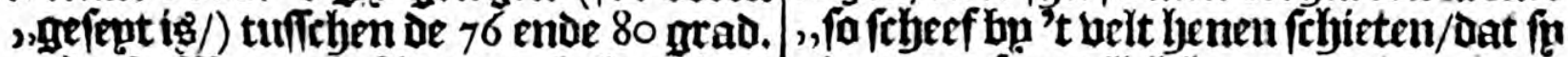

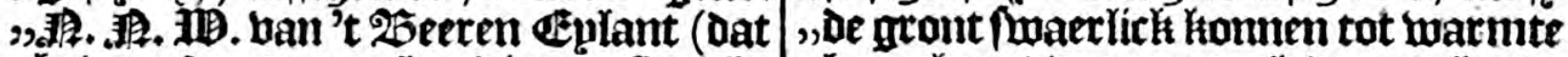

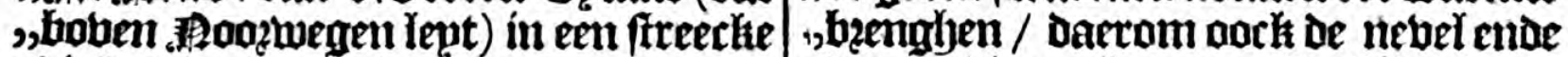

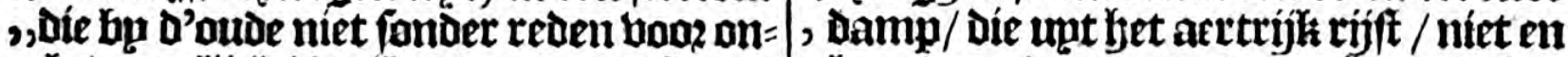

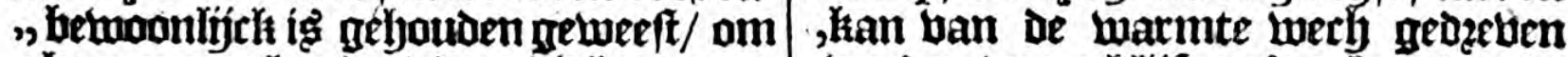

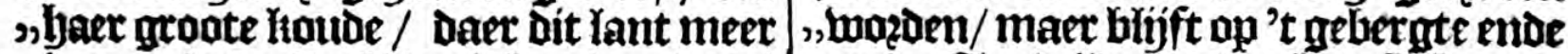

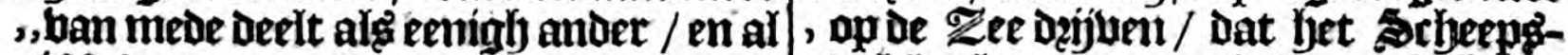

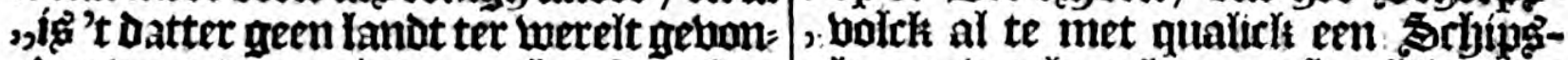

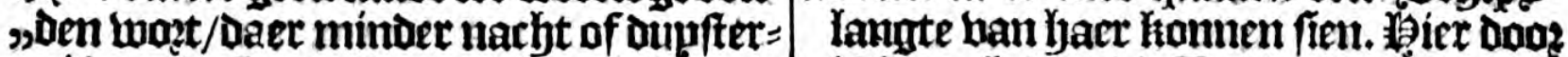

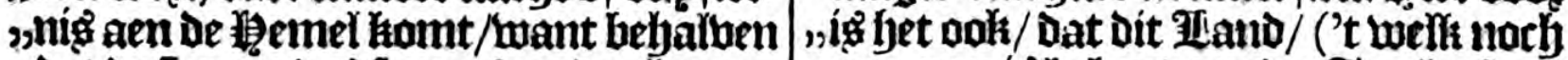

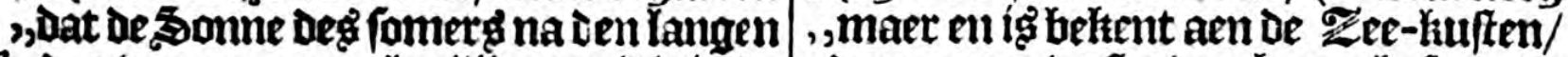
, dan, Dag van 127. mael triiii. uren (Die in be

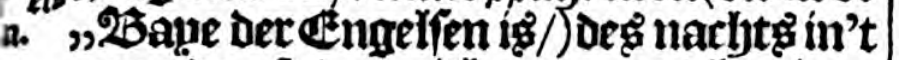
"noozben fo heunich onder dent Yortizon „Daelt / Dat bet licht in't geljeele somer= "frbe balf-jaer geen/mgen kam ban Den ,"Yligemel wedy gaen / enoe heljalben bat= ,tet in't tDinter ficthe half-jaer thee bolfe

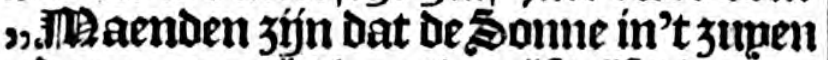
„Daugen maecht ban twaelf/elf/tien/en= ,De fo boozt tot een uren langly / fo komt 2) Jph noct//felf ,in't 3 upen is/te weten in 't yartjen bam ,be lange narbt aldaar op $12 \frac{1}{3}$.graden na ,aen Den harizon / fo dat daer ter plaet= sfen / te Leten op 80. praden / nocth alle "etmalen 't fítht des daferaet aen den

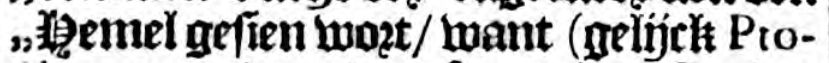
„2lemeus ende andere feggen) iun fien den „Dateraet alç de Eonne noch I 8.maden .

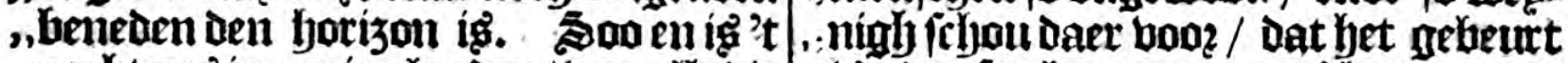

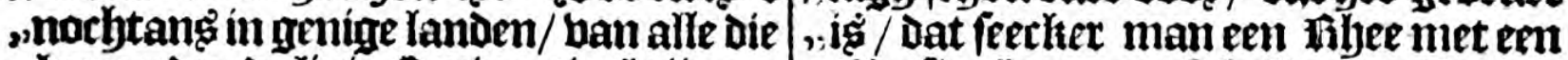

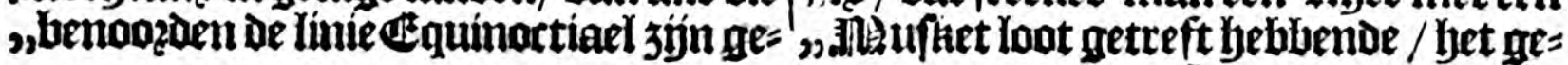




\section{8 \\ Derde Schip-vaert van Willem Barentfz.}

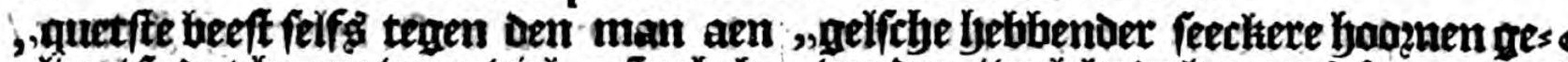

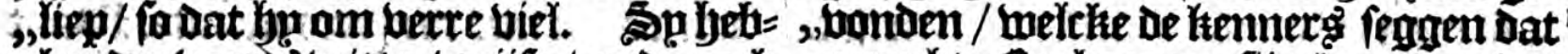

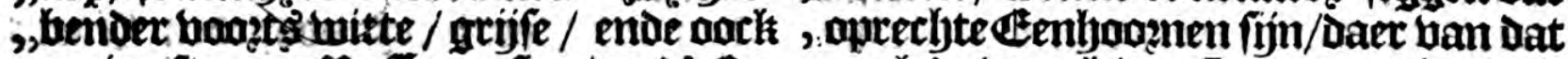

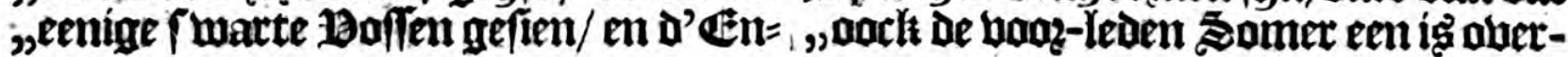
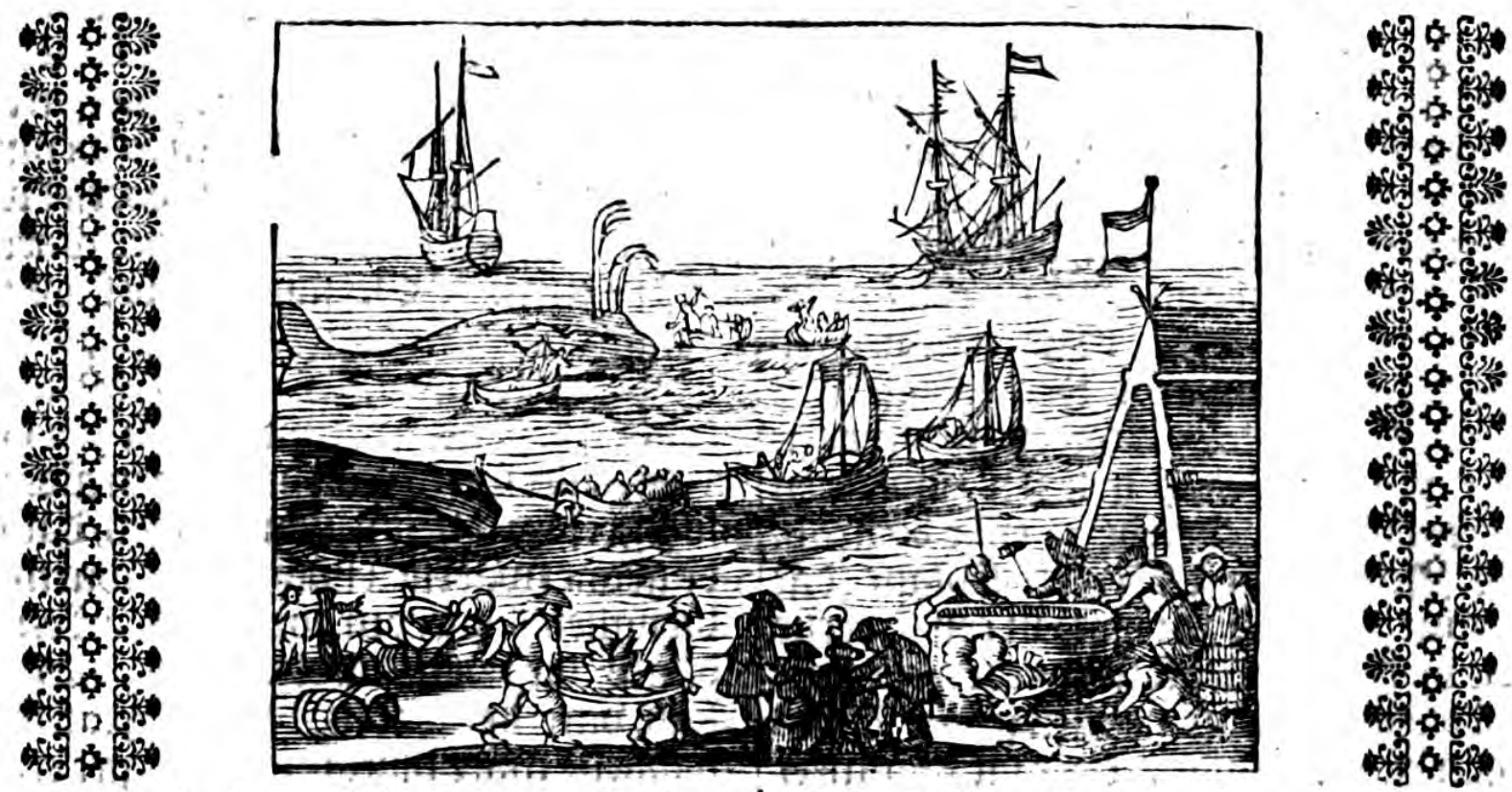

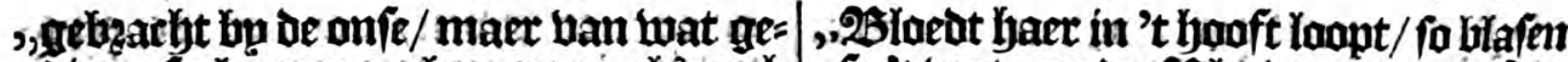

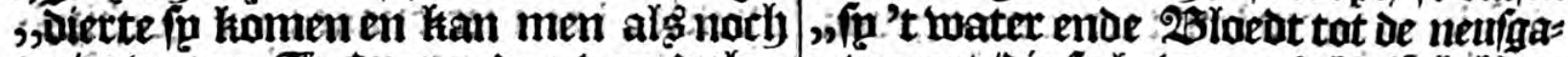

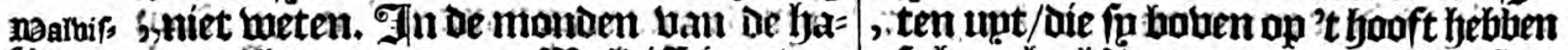

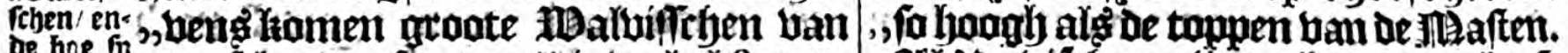

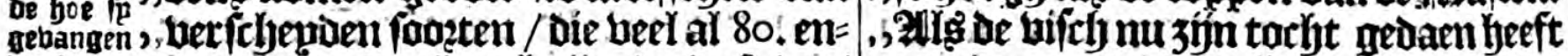
wozoen. , foe meer baeten langh zijn / ende fo bet/,nae de gront / ende bem felben moede , Dat lyet fpeckl Daer af komenoe meeft al , gemaeckt/ fo palmen de faloupen aen/ i. betfoden fan wozoen tot oraen. Daer ,ende febieten hem met febichten in'tial

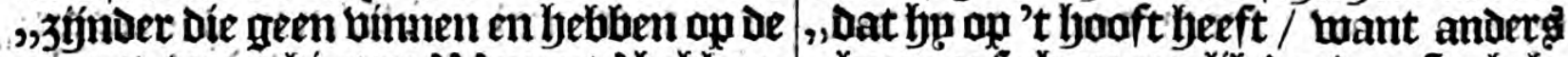

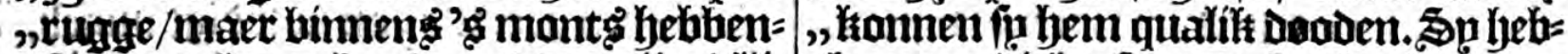
"fé groote lange haerden / dat sijn blij=, "hen een dicke f watte humbt daet een „, men die de groot fte een baem lang 3 inn "blief Ken ober heen loopt fon that ende

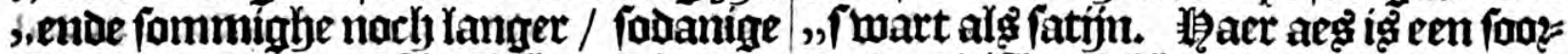

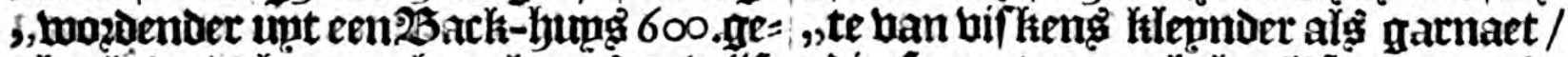

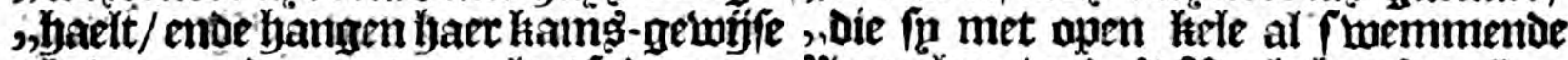

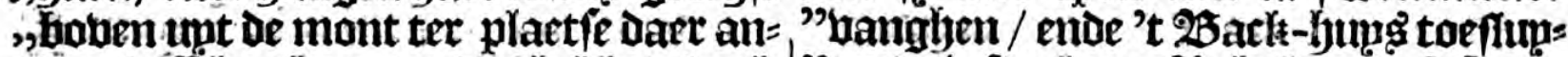

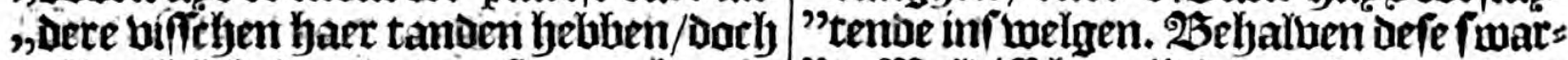

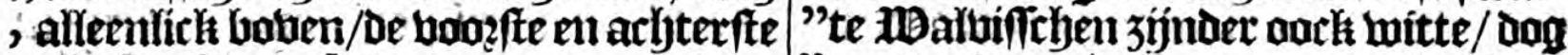
,jún feer Kklepn/po datter maer 400, boo? "en wazien niet boa? gaet getjouden.

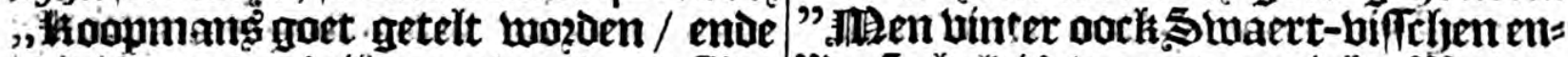

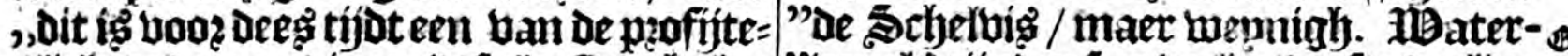

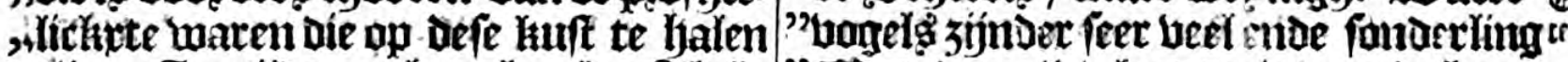

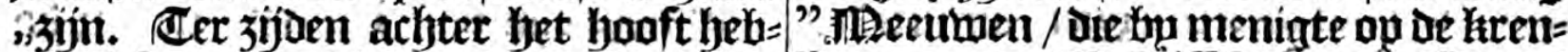

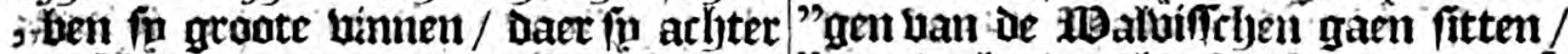

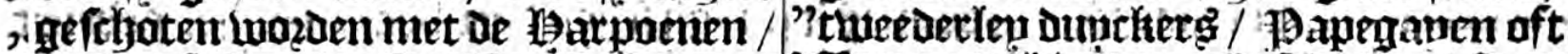

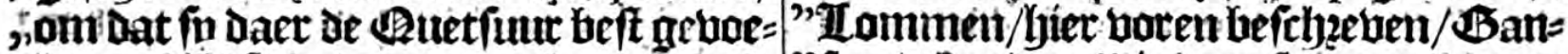

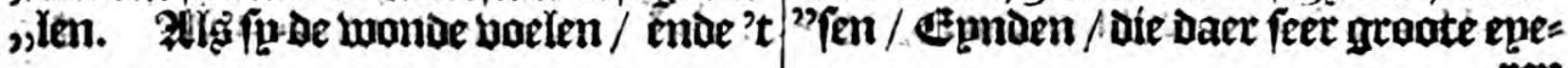




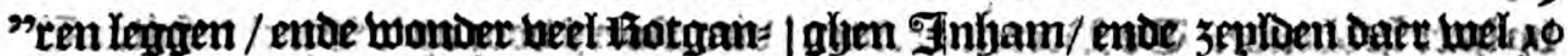

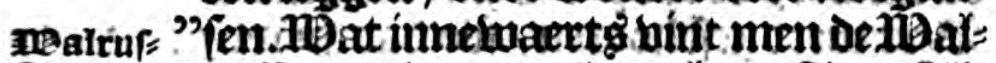
זen. "ruffen / Die up wetmochten Zee-Bit: "pfyanten naemen/om dat in in meoote "ban fictharm en ie tam den dene

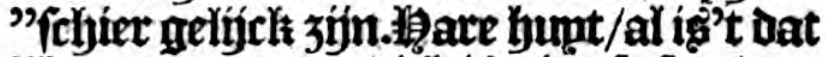
"'gn untermaten dick is / jae fófer dat= "men t'Almfterdam een gefien heef than "400, pont f'waer/ig nochtang mepnig "weert/om Dat hn te blarighig. Sou heb= "hen feer beel speck om אilraen af te

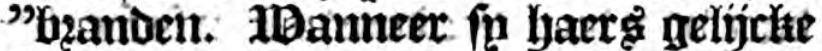
"fien Doot weflagen Yeggen/fo htimmenfe "op be doode bu futecken menighte dat ip "die Daen bzoenenende verder ven. A Bijf

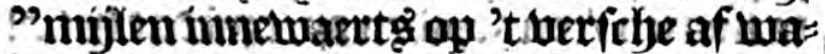
"ter/leggen De zee-jontien feer vameenet "aert met de onfe. Enve dit is innerfte "Kermifie die wo van dit lant ende fijn "gediette tot noch toe bekamen heblien. Ben 23. Itmí voetoer een beel ban haet bolch aen lant / m te fien boe beel

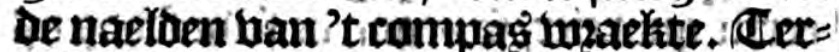
wifiten quam daec een witte beer na' 't

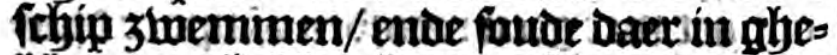
hlommen heblhen yadoen fu meen geraep pemaecht / fo febouten nae hem met een raer / waer booz th weder af 3 wom ba 't febip nae 't enplamoti daetr d' andere

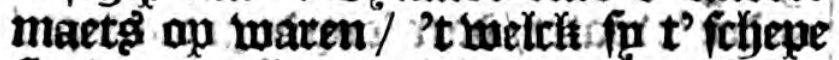
fiende na 't lant toe 3enlaen / ende feet beftonden teroeven / fon dat d'andere metuden fo fawden' $t$ Felip op ren rilip

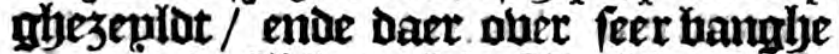

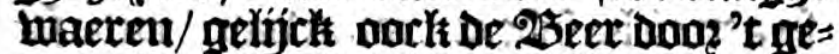
roep feer ver fetyeickte dat fu mederom

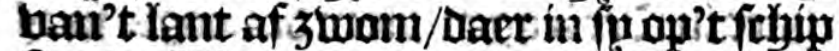
feer betbliht waeren/obermit'se maet s" dié aen lant waren neen reiverer en had= ben. Plengaente de watatinge ban't rom: pas / werot betwonden dat het fecheel= de 16. graurn. Den 24: Dita raevden fin Een troat ftuli immetwaerts aen $/ \mathfrak{e n}$ te lauiDe ghegern zünde / vontuen aloaer twees

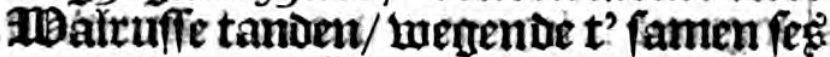
ponot/met noely eenige hlephe :Den 25 .

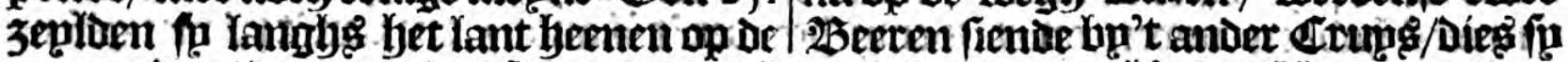

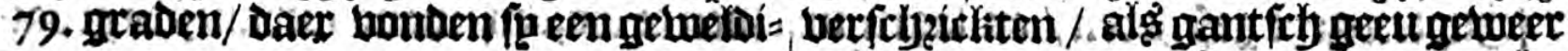
mijlen in/ dan moeftender thederam tugt taveren bermit contraxie bint, Đea 28. raechten f́n Goben den ljoech ditaca de weft-zijoe laugh/Daer de bonlyets in for meonte menighte waren/ Dat í plot fétick tegthen de 3 eplen aen blogen. Ben 29. maeften in bam 't lanot af wijectien on Degi ng wiffe / ende zeplden tat op de 76 . maden 50 . minuten. Den eerften Gulp

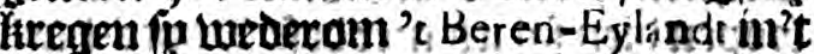
welichte/ ende daen quam lan Cornelifa

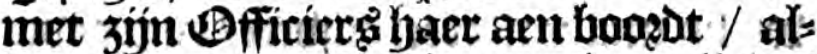
waer fu ban rontrarie meyninge zijnde/ epndelith $t^{\prime}$ famen verdoendien dat elck fin contug foube voligen/ende lan Cornè

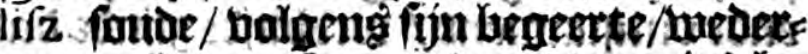
om seplen op 80, graden/ vetmit by hem in-beetbe aen de ooft-zijoe van dat Lanot fíchteliéti dop? te futten ralten

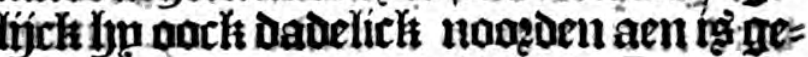
gaen: Taev-en-tegen Willem Barentiz. 3unt aen/ uan wegen' $t$ yge. Ben 1 x. Dito

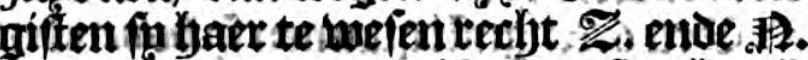
bam Candinoes, dat is de oofter-fyecti

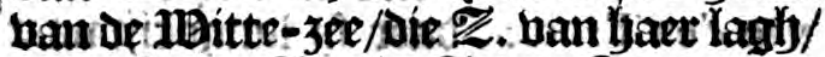
ende gingen $\mathscr{Z}$. ende $\mathcal{Z}$. ten 10 aen op de hounte ban 72 . Mtaden. Den 13 . bebon=

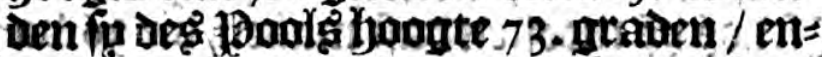

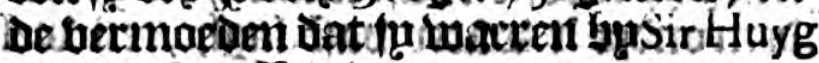

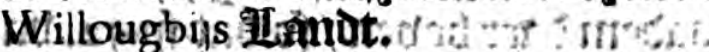

Detr ic 7. Gludy twaten furap de foongte

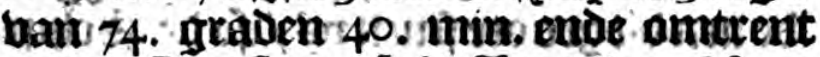

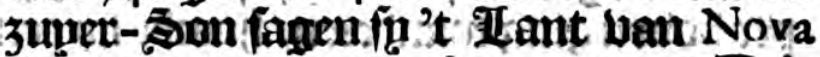
Sémbla omtent Lomsbaey. "Den

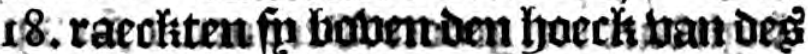

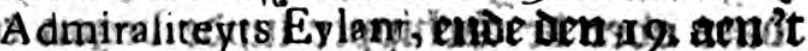

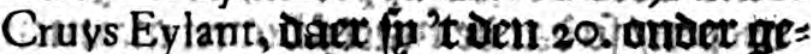
fet bebthen 7 bant ftonden niet berder overmits bet ng. Sop racyden toen met haer achtenaen lant/ enoe gingentuae?t

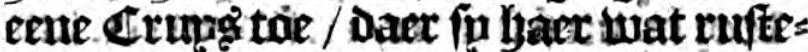
tem / am bonat nae 't ander. Erupg: te

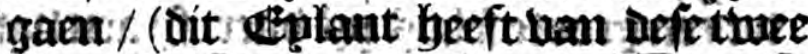

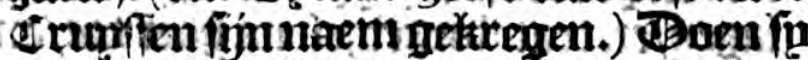
mop oe wegh baren / wetornfe twee 
by yaer hebbenoe. To 25eeten rechten fich tepl bp't Irung op/om't boick wel te befien / want fp ruge kten beel nauber Dan fo fien / ende nuamen boost daer op

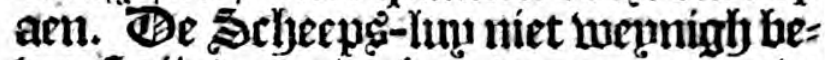
beeft zíndo / ginghen te rưge nae de fofyunt toe / al te met deerlije am fiende

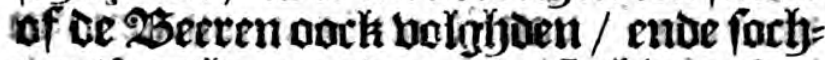
ten t' ontloopen / maer de sorljuper we= derfielt fe / fengenie./ die eerft begint te roopen dien fal iek met defe 2 boot in fin bumbt fteeken/ want't igi beter dat wo by malkantieren bilíben / en proeben of wu haet inet ont roppen berbaert mogen marchen: Son gingen fon dan al fortjengi enoe geftaoigh na de foljunt toe/ ende onitquament/ wel blijoe zijnde dat fi 't vettellen morlyten ende ben katten= Dang foo ontipzondjen waren. Den 2r. Fulu wert des foonla boouthte be= bonben te jojp 76. maden 15 . minu: $\mathrm{ten} / \mathrm{ende}$ oe af wijectínge ban de naelue 26. graden ftíf.

Den 6. Ougufti quamen fú fooben Den hoek vann Nalfouw, min ben 7 , bp de hoeck van der Trooft, daer fí lange tijt naer

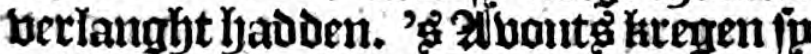
miftigh weder/fo dat po het felju aeneen

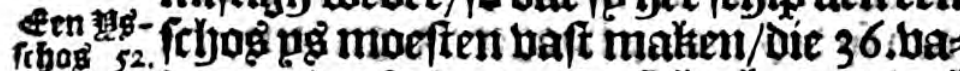
badem Dem onder't luater baft lagh / ent twel aich. 16. vadem daer boben / fo dat pi 52. ba= Dem dief twas. (Jen 9, dito nach leggen= toe aen Dit mo ote fuck ug / pa de schip= per boben ging imandelen/ hoozde by een Berf fuuben / en bututen boozt fiende / lagh daer een moote 2 seer aen't Srhip/ Daer ober de Schipuer lupde begron te roepen/fo dat oe maet to bonen quamen/ en lagen den 2 beer aen de fefunt legen om net fink kaunen daer in te klimmen maer pp maekten een groot petaep/ Daer boog bp verficlyziclite/en f wom een ftuch weg / Dan freerde terftont Lederom achter een groate fohos pa daer fo aen baft lagen/en trat ftoutelijek na baer toe/om booz by't / flyip op te falimmen / maer de maet baoden boben op 't Schip bet flbuuten-zepl gefichoren/en lagen met

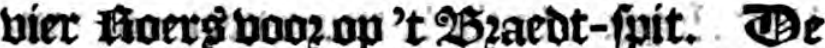
2Beer wett gef chooten/ foo dat lyg weuh liey/ Jet fneeude fon feet / Dat fu niet fien konton waer hy berboex / bach bermae: den dat ly acliter een bogen hew bel bleef

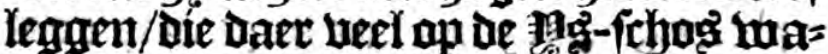
ren. Den 10. Alugufti began't digh te défuen / en in bemerckiten baen noch erif toat het moote ftuck pas / Daer fis aen gemeert fagen / baft aen de gront wagk/ want het ander pog dzef al te mael Daer boos bp: 1 ier booz waten fu feer be= bzeeft dat fe in 't pis foude mogen belmeit wozoen / Deden derbalben groote naet= ftiglyent / arbent ende blijt om ban daer te homen / en zequtoen op't ps bat bet al kraechte watter ontrent was / rakenoe noch aen een ander fobog ngi / daet fin't wederam baft aen maerkten met het werp-ancker dat fo daer op brachten/en bleben daex leguen tot den abonot toe. Zutip nu' abont gequeten fadoen/in't eerfte quartier/fo begron de felbe flyog gg

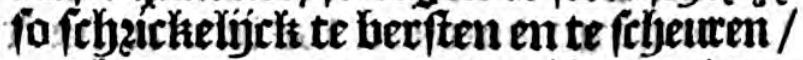
Dat het met meen woogen its unt te douts: ken / folagben met de fteben daer aen/ bierden daetom baer toun/onde raelten alfo daet af : Jet folyeturde met een epfies

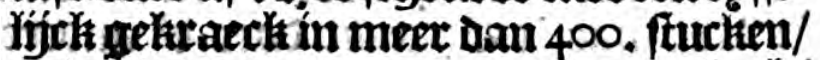
onder't matex/ oaer' $t$ op de gront lagh/ was ro bavem dick / enve hoven water twee badem. Ban daer quamen fp twe= Det aen een ander groote fchos ps/Die feg badem onder water laug/ baer maekten ig aen elche fude een toun vaft / ende fas gen doen noch een ander fohog wat bau

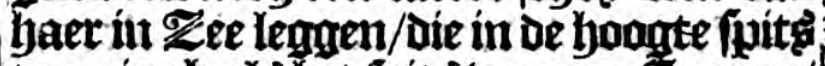
toe-pingh ald bet pit ban een /f noten/ daer boeren fo aen / ende bebonden dat let wel 20. badem diep aen de gront laa/ ende rupm twaelf bolen water. Den xx. Alugufti raepden pa wederam aen nock een ander fohog ps/oe weleke pa be= bonden 18 . vavem onver water baft aen de gront te legen / ende 10. Daer boben. Den 12. zeniloen in nach naetder on= oer't Tanot / om wan't pis niet gef fyo=

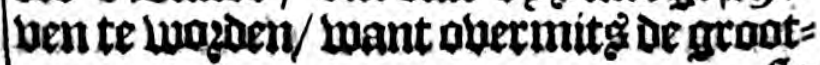




\section{Om't Noorden na China.}

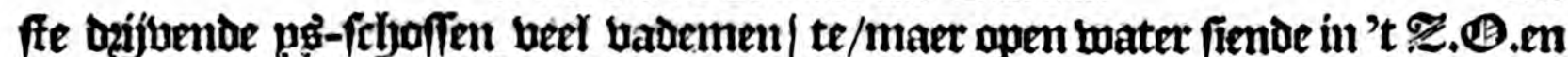
dizp ozeben / pou waren fin dicljt onder't Tanot op 4.Oft 5. vadem beter daet ban hef chetmt : THaer wage en groote a fwa= teringe ban't geberghte/ ende f́n maek =

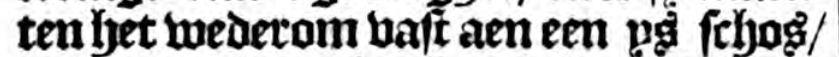
niemnenende noemden dien baeck den Kleynen Zat-boerth. Ys-hoeck.

Den I3. Zlumufti 's mozreng quam= 2Reer ge: Der een 23 eer om den Goft-baeck ban't Doot. 'aant benen dicht by' $t$ sebip/en een ban de maet fot boot bem' $t$ beenin ftucken/ maer hy buppet de norly met find dąie po: ten op een bergh / boclj fin liepen bem na en fiaeglyen bem dont / vittoen bem / en

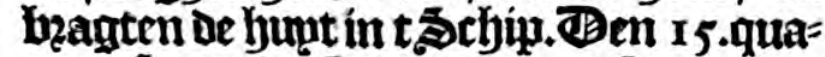
men f́n aen de Eylanden van Orangien,

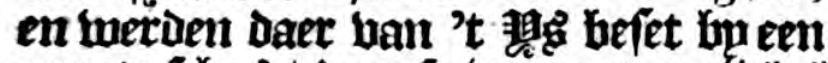
meoote frbogs / daer fo in groot perifecket waxen am't Schip te berliefen / cork rackiten met moote moepten aen't laut ende de wint quam van den $\mathscr{Z}$. (1). waer

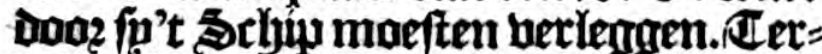
Barthent wijle fin daer mede befigh en feer lunt-

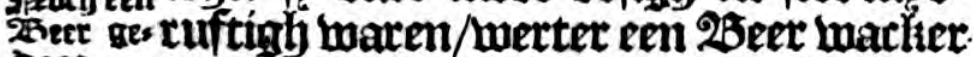
boet.

Die daer langen fliep / en trat tot yaer aen na't Sachip tae / fo dat pu haren ax= bept maeften ap fchoeten / en fich tenlyen Dent 2Beer te weer ftelfen/Die fu bane't Yijf

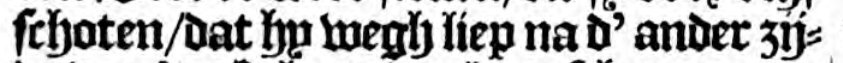
be van't Eptant / en begaf Yyem op een febos $\mathrm{pg} / \mathrm{maer}$ getwaer wer bende dat fi nae hem toe raepoen (fpzongh ha weder in't wateren finom weah nae 't tanot toe/ Dach fun onder felbepten hem den weg en hieumen hem met ten bijt in de kap / maer by doack $t^{\prime}$ etckend onder ald fip met de biff naer hem toe tieumen/fa dat fo moate moente hadoen eer fó hem boo= Den franden. Tien 16 . Dita raenden fip on= Der baet 10. met bet Facht nae 't bafte Ianot ban Nova Semb!a, en yaetoen de fthunt baben op't ths om hoogh / Klom= men op een hoogen bera/en peptoen bae de Ys-haven teetamen waren/ begon't

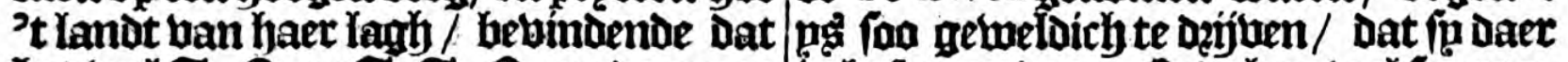

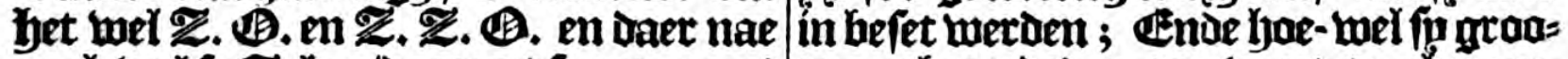
noch wel fo $\%$. lag / Daer unt for een quart ten arhent deden om boozt te gheraes:

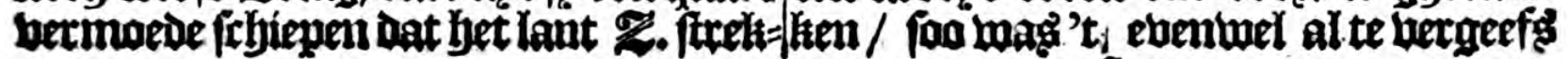



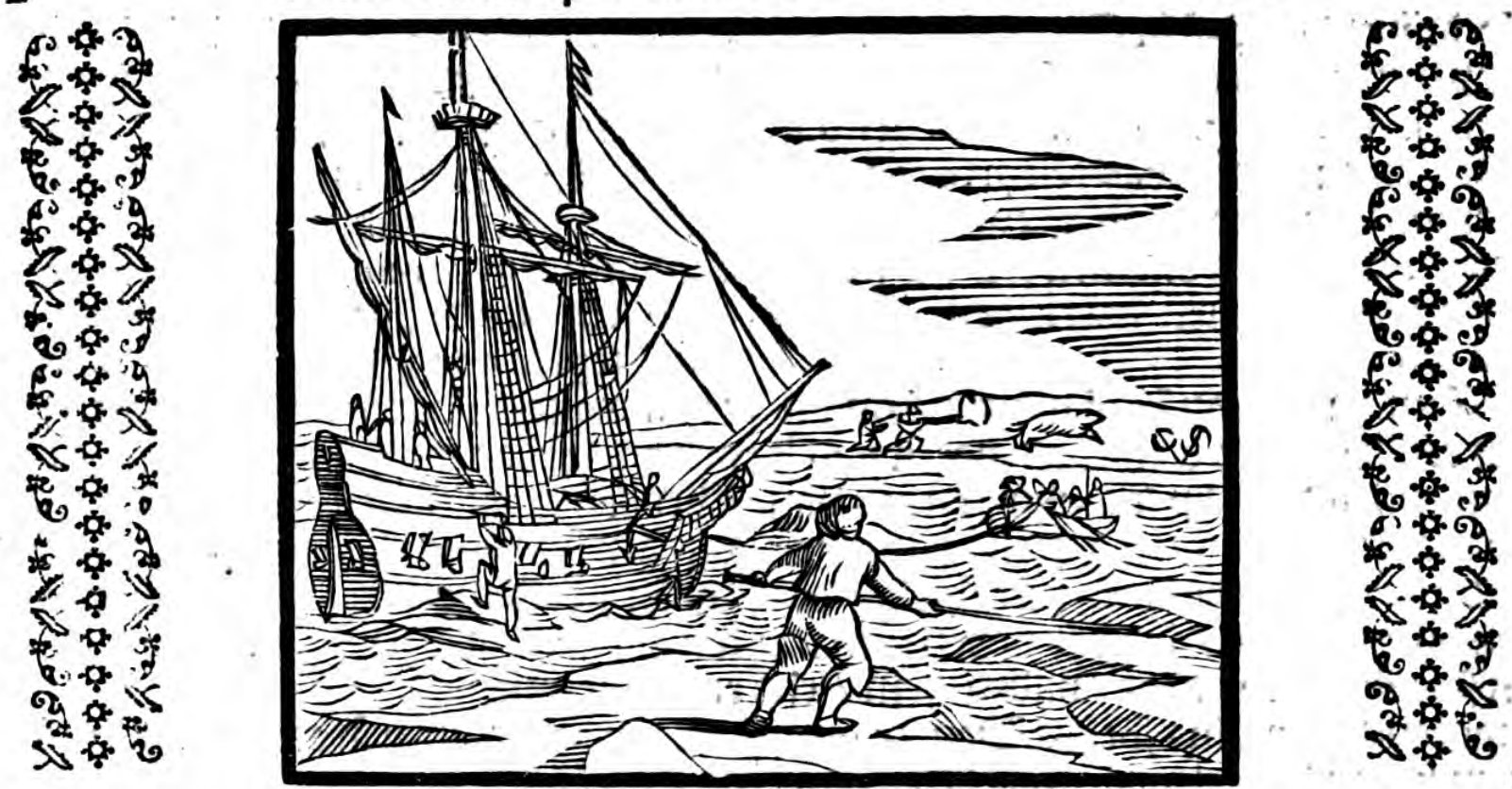

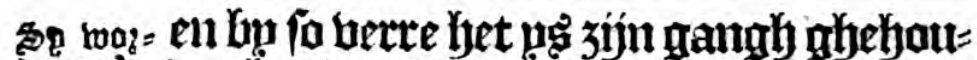
den in't den fadoe/ fi baoden datmael 3. man= ende obe= nen luetloten/Die op't pas Laten am ope $=$ bivongen ninge te makten / maer alfoo ju te rugoge

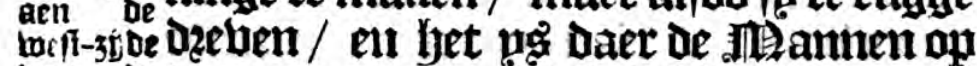
ban oe waren mede dzeef / fo bebien fo gau ter

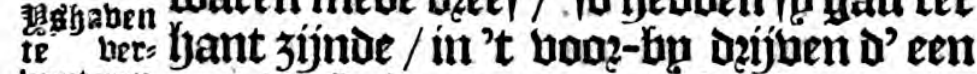
bunteren aen de fmijt gegrepen / d' ander aen de fehoot/ent de derde aen de gronte brage die achter uut fingly/en quamen alfo abon= tuerlífelz met fulchte flinger-flagen/ die fu waer namen/noch befouten t' (cheep/ Daerfe Godt montelijektio af Danckten/ want het wage veel eer geichapen dat in

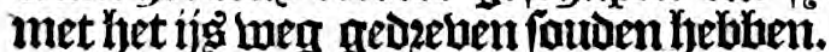

Gp den felwen dauh quamen in dege about aen de weft jijoe ban de Ys-haven, daer fu boosta de geljeele fande whter met frooter armaede/elfende en= de berdiet maeften oberblíjuen. đBen 27. Deerf fiet ije ronom 't fohip / ende alfou 't goet weer lwage/gingen een deel ban de maets aen lant: alls ip nu een fuct weegh gegaen waren/begon bet tame=

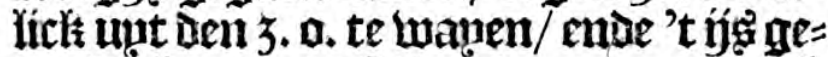
weloigh aen te fetten boas den borath Iej) am lyoonly / Dat lyet achter fat als' of 't inet den aer inde gront gefeten hasde/ ende fiben dat het daer foude vergaen - Gebben. Tie in 't Schip waren fetteden

fluct ien bock int am fraer líf te bebou= den/ende fíeten een blauge wanen/ om D' andere die aen lant waeten te locken na't fichip te faeten. Defe fiende de blag= qe wanen enve bat het fehip alfou op-ge: ithoben was/hebben ficly al wat fo mog" = ten dermaert gehaeft/mepmente bat het

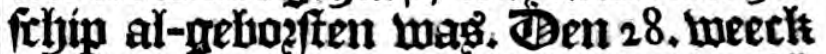
bet ýs wat weg/en het felýp fettede fich wederom recht / maer eer 't noch recht ging fitten/wag Willem Baren ifz met oe antere Stumman booz den boegh gaen befien lyae't al geftelt/ en bae beel het ge: refen wag/ ende terwijl pa Donie waren ober fmíe ober eflebage om't felbe te me= ten/ fo berfe het Srhíp ma met fodanigh gekraek / dat fe meenden baer líf quít te zín / niet wetende waer pu haer berghen

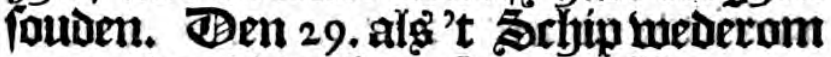

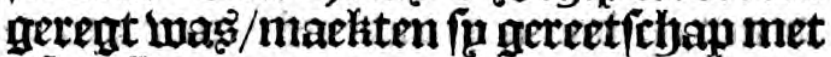
piere late-boeten en andere inftrumen=

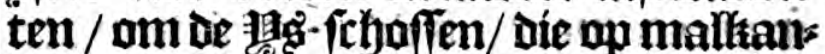
Deren gefeljoben lagen te bzeken/maer't

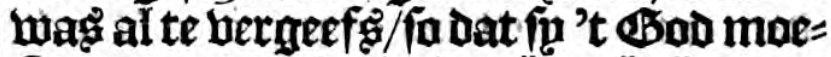
ften op-geven / ende ban bem hulpe ver=

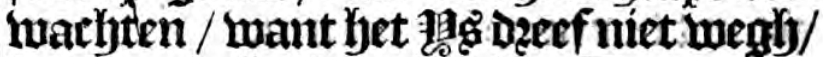
Dat lyaer hutpe doen konde. Tien 30. be=

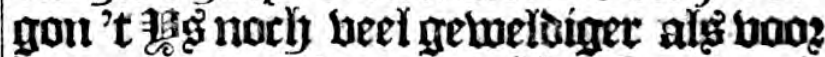
yeen op den anderen te felyuplien tegen 't Srhip aen / met een frachtige wint/ enoe gronte jagt-pinee/waer booz't gant: 


\section{Om't Noorden nae China.}

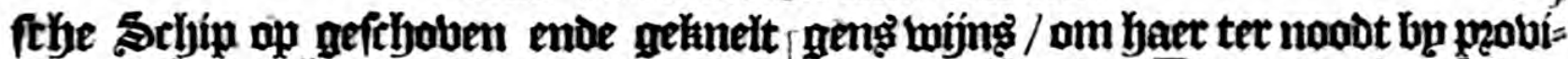
wert / poo dat het alleg begon te kraken enoe te herften watter om en aen was / ende ficheen aen bondert fuctien te fplij= ten / 't welck fo feljiclizelijels am Gooren en fien wage / dat een de lyapren te berge ftonden ban't afgrífelick felyoum-ipel.

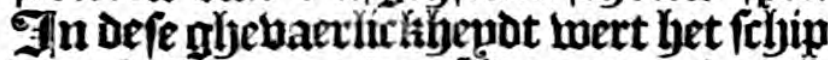
boen't ifsaen weder-fijoen tegeng mal= fanbereti aen quam / recht op in de booglyte gliedegen / ates of het met cen bífil on-thebifelt ware geweeft. Ten 31. werden fa wederam bons 't ghetwel= bigh dijjoen wan't ýg wel 4. ofte 5. boe= ten op-nbefifjoben met de boos-fteben/ ende 't acliter-fobip fat in een kloof ijs/ daer boo? fn mepnoen / het roer foule ban 't fethumuen deg' ijgs gebeift getweeft zín / maer back ebenwel met de pen in ftudken / en foo't achter-fchip in 't day'j= benue jós geweeft haude/gelijek alshet booz-fehip / 't gan/che booz-Flyip foude op't ijs gefichoven geweeft zinn / of t ma= gelic inde grant getwomen bebben/waer ober fo gantich bebeeft waren / ende hadoen baer feluut en hoot al boos upt op't jíg gefet / om ter noot lyaer te ber= ghen. Allaer omtrent 4. uren baer nae ig 't j́s ban felf fo fo berblijot waren als of jp haer le ben gevomiden hadden/oni dat lyet Sclyip nu wederom blot gewozien luag. Sp ber= maechten 't roer met de pen lueder/ende hingen bet unt de faec op/ten epmide/of in weder alfo geftholuen werden/Dat het Dan foude glyebijt zijn.

Den eerfen כeptembagig hegon 't íg: meterom te felyuphe / alfa Dat het Bchip ober fín gantictje líf wel twee boeten op-filjoof / maer bleef evenwel noch al

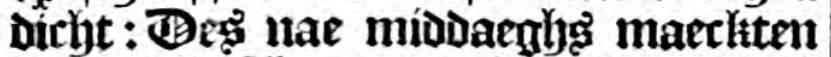
fo ghereedt frlyap om de filjugt ende bock aber 't ijga aen lanoet te fiepen. Ten 2. be= gon't 3 cljup wedetom te fclumben ban $t$ ígs/krakende ende barften fo gelueloigly/ Dat fu raet/aem bonden/niet tegenftaen= be quaet weer/se fichunt aen lant te been= gen met 13 , tomen boot ge en twee vaet = ire $t^{\prime}$ moer bouden. Den 3. werden pu we Derom gaende ban 't jyas darx futegeng

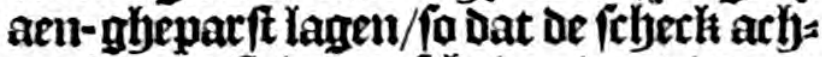
ter bam de fteben gefichoben wert/ maer de plancli en Daer t Schip mede ver= bunot wag / meynden f́ noch / Dat daer aen bleef banghen: 1 et toulm te loe fogalt mede fucken/met nieu kabel toum dat jo op 't íj' hadoen baft gemarckt / boos 't gemeloinb aen-parien ban't ij:s/maer 't sechip bleef noch oirht/'t melek te ber= monderen wag/om dat lyet ýg fo gewel= Digh Deef / jae Datter Hgibergen beeven foo groot als de \%out-bergen in Span= gien 3 ín/en dat omtrent een raer fiboot

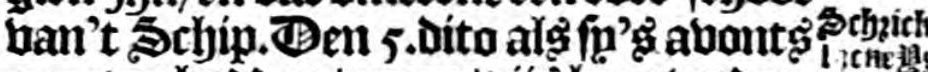
gegreten hadoen/quam 't íf lyaer weder= bersen. om befetten ende berden dauper ge: parft/fo dat bet Beljip gautffly feer ober zijd begon te hetlen/ende beel leet/maer holef dow Godeg gentade noch diclyt. In fulclten noot bonden fu raet faem / baer oude folt / met hrtugt/ loot/roers/ muf= hetten / ende ander nelweer aen lant te bengen / om aldaer een tent of lyutten omtrent hare frbunt / die fu aen-ge $=$ beacht badoen / te inaken / namen ourf

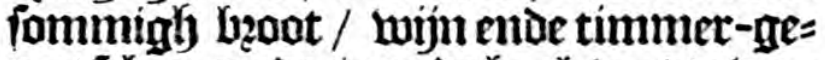
reetfichap meue / om de back wat te ver= maken / of fo lyaer indan noot mocht dienftig jín. (B) 't sebip en wag foa beel water niet / datmen cen putec bol had mayen felyexpen. Den 7. gingender bijf ban de maets' op 't lant / maer de thuee freerden weder / ende deie gintren baogt ointrent een paer mijlen te landes

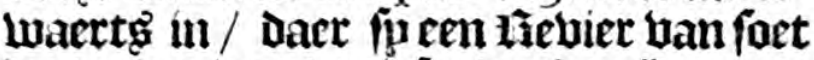
water bonden / nitigader soclt groate menigte ban ljout / Dat daer wag fiomen Dzijben / ende eenige boetfappen ban

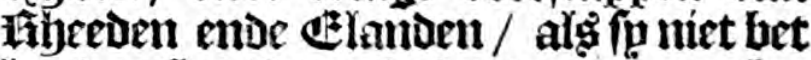
fonden fien/ Lant de boeten waren glye= Gilooft t'ecne urooter als d'moere / daer Dode fy fulcr Jermaeden. Jen 9. Dito qua= menoer ' nacht thee 23 eeren dicht by

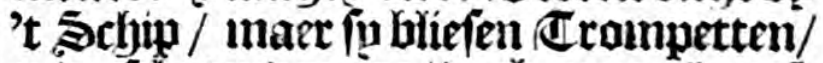
enue frjotender nae/Docljen raeclitemíe niet/ 


\section{Derde Schip-vaert van Willem Barentfz.}

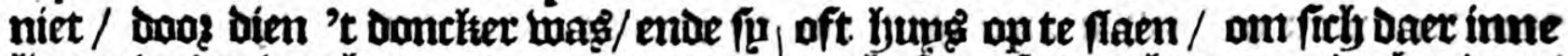
liepen weder wegly.

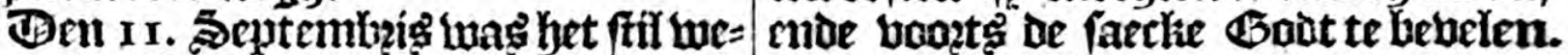
Der / enoe fo gingen inet lyaet acliten aen liex toe heeft fiely eell goede ongebaen=

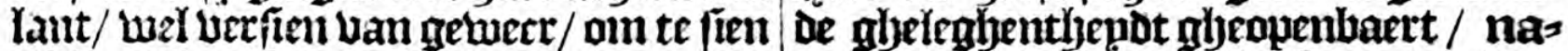

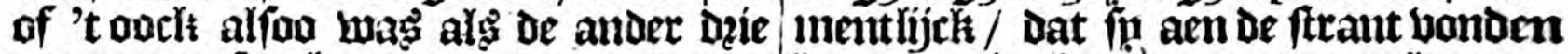

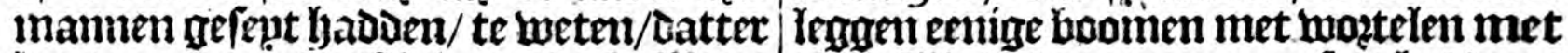

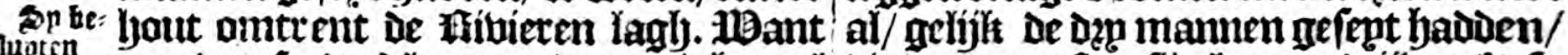

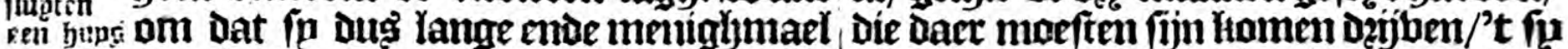

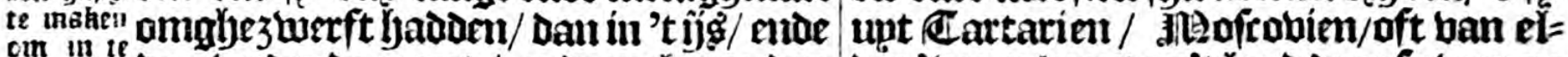

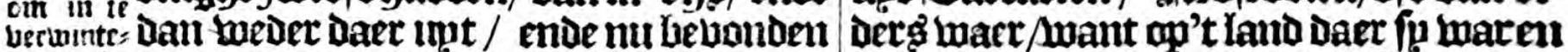
ren/ cnoe bat fp daet baft bileven fitten / ente niet gantich geen boomen en waflen. Dau

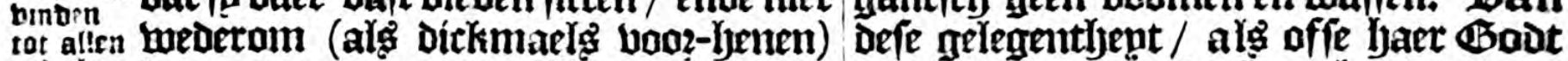

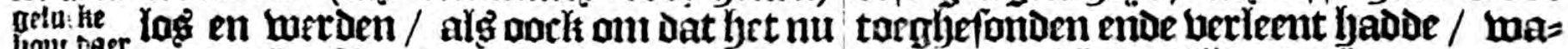

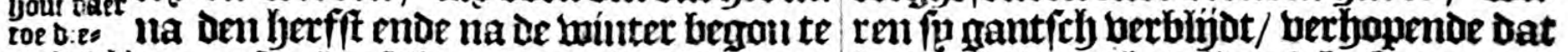

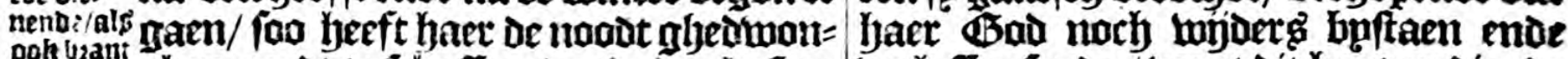
bour/om ghen raedt te icljaffen / ende den beften berlofien foude / want dít hout en diende

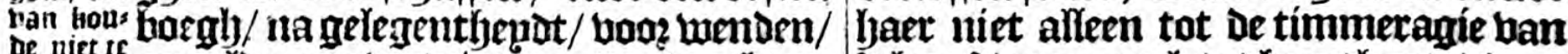

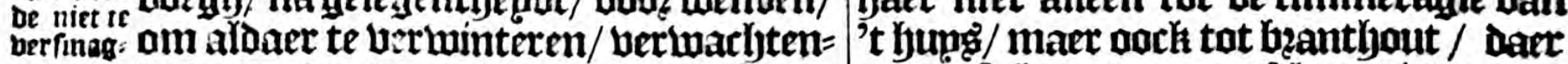
ten. de wat abontuer Bod berleenen foude. mede fo baer den gantfijen winter ont:-

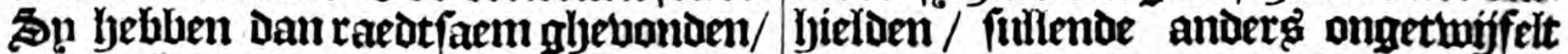
on te beter boo? de houde ende wilde ban gronte houde moeten bergaen beb= beeftei beflyerint te blíjuen / een hutte ben.
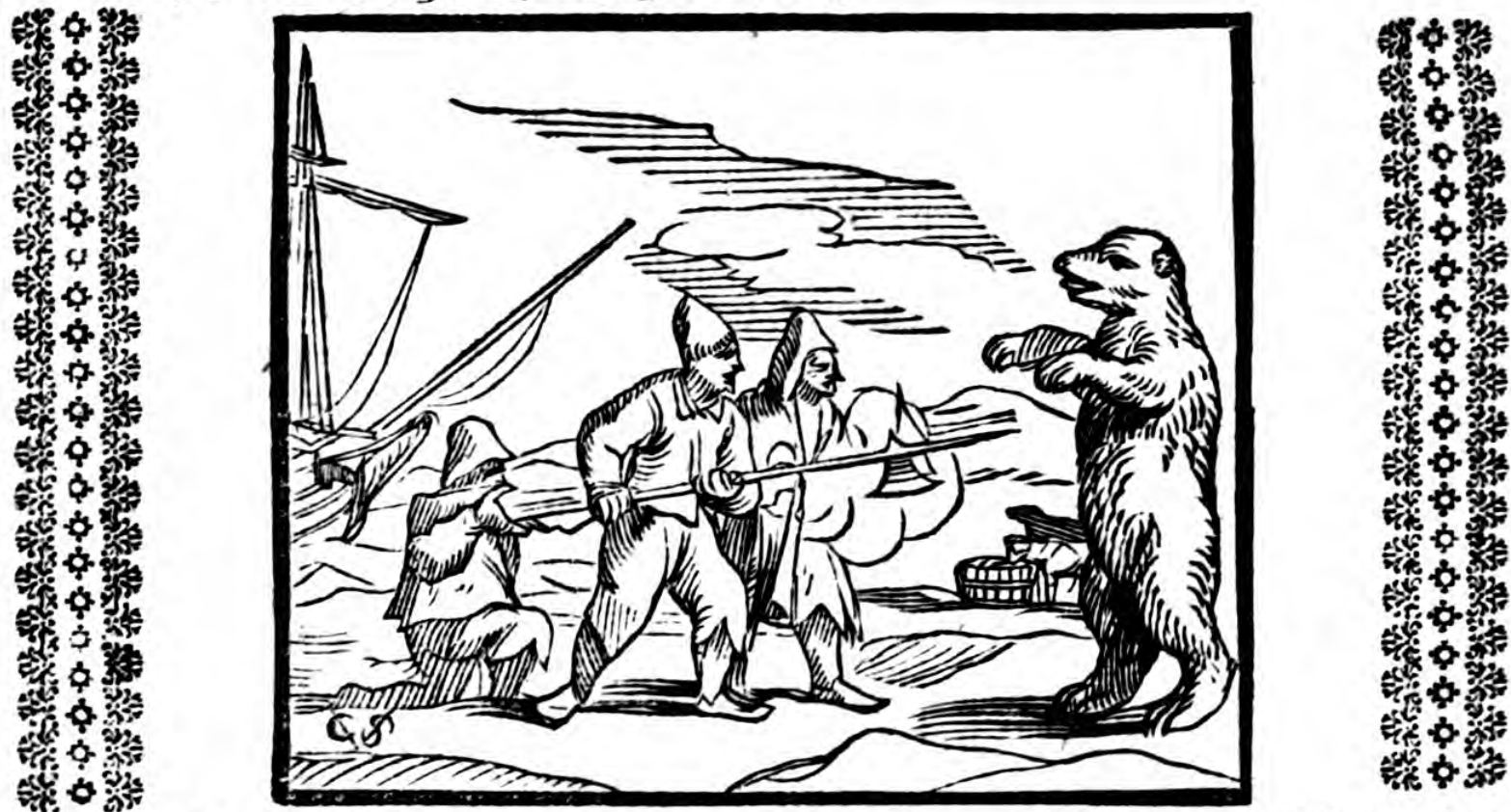

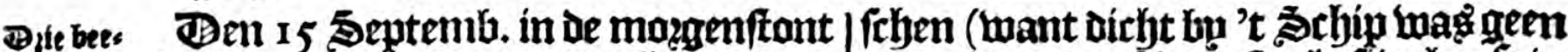
ren ho: gincker een man ou de wacht/en daer water) d' eene 2 Beer ftack iṕn lyooft in

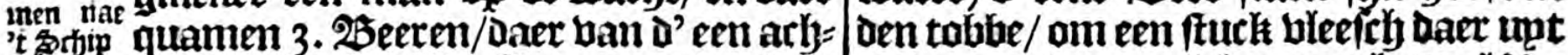
toe i enae ter een fturk płj bleeflergen / D' andere te nemen / maer 't bequam hem als de

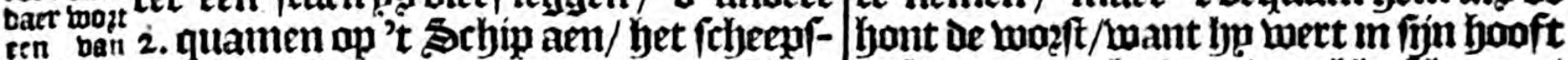

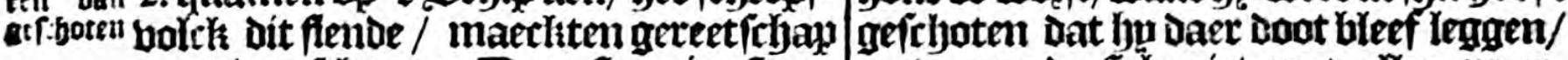
om die te fefleten. Taer ftont jugfteen en berroerde fich liet met allen meer.

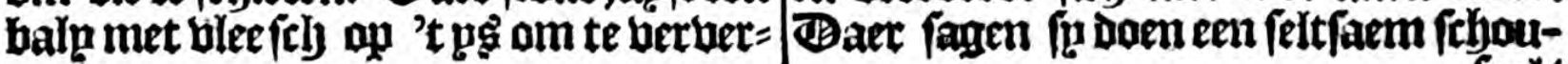




\section{Om't Noorden na China.}

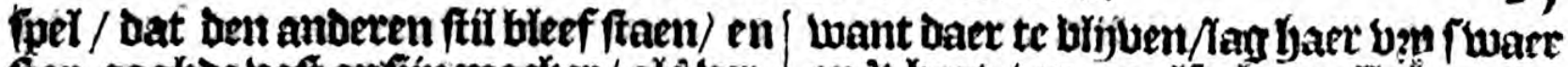
far-oogtjoe baft oplín macter/ als ber= wondert zínde waerom by fo ftil mocht bijiuen leggen/hy rook daer aen/en fien= De dat bu nu boot was / ging ten laet fen wegly / maer de maets loerden baft op hem/om te fien of Jp oock weder homen poude/ nlyelícels lyp dede/en gingly op zín arljtec/te pooten ftaen/om alfoo op haer aen te fomen/Dan tertwílen by alp ober epnot ftont / foo feboot hem een ban't bolck doos den butuch / Dat hu wederom op fín bier boeten neder biel/en met een groot gefiríf h wegh liep. Den Dooden Seer imeden fouen bupk op/en baelden't ingelwant daer unt/en fieloen bem ober epnoe op fín bier pooten/ oun te laten bebiefefen/ban meeninge zinde/fo fo met het sclup log geraccht ladoen/ hem in sollant te bengen.

Dactna begonmen figen fiete te ma= hen om fot bout te fiepen/Daer fo't but meenoen te fetten. Op die tijt benozt in Zee wel twee bingeren dicls. Den 16 De= den fo d'eerfen tacht om bout te balen/ en beachten dien oag 4 . ballen ober't

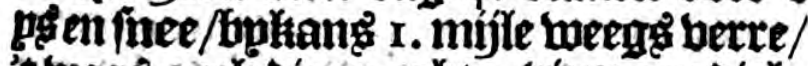
't bzoos ooct dien nacht 2 . bingeren dick. Den 17 .gingen fu met I3. mannen na't bout toe / en trocken onser yaer thienen 5. en 5. elche een flede boots / d' ander 3 bleben fog 't lyout om dat te belyoumen. Sploeden gemeenlífelt 2. tocljten Dargis/ en ketegen fon't lyout ouer lyop Daer pu tímmeren wiloen. Den 2 r. wag het fo kout / bat pip de tombung helleden in 't rumm moetten bzengen / am dat het bo=

ae rima ben alles bebzoos. Den 23. fterf haer merman Rimmerman/en fo beuraeben fism den 24. onter de Cingel in een kloof baneen bexgh / ontrent een af-wateringlye / om bat fu niet in de aetde en konden dooz de

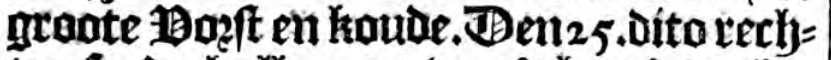
ten fig de balken op ban't lytng / en be $=$ yonnen baft te timmeren / Dan fadde't Schip logigewozoen/ fouden fyet timme= ren baeft beblien laten faen / en geteet

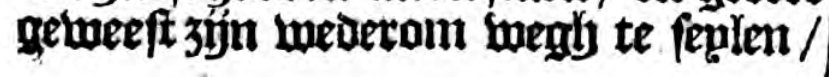
op't bart / maer alla baet alle bope be: nomen waje / foo moeftenfe ban de noot em beuglyt malten / en met lj́t faemlyent berwarbten loat inthomite (15ad betlet= nen foudo. Jen 26. was 't een wefte wint en open \&ee / maex't Soljip bleef ebentuel baft leggen / al fon dat liet haer meer berdzítet Luag / alta weugljoe. Sy waren boen nocly 16. All an fterck / m onder dat getal mafier al-te-met loch

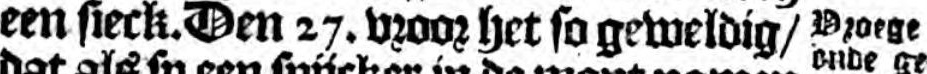

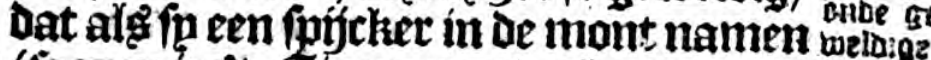
(fo men in't fimmeten beel gelwoon ig gojit. te boen / fo bleef't vel baer aen yantren/ als men die weoerom unt be mont nam/ datter't bloet na volgboe/) ja't wagi fa

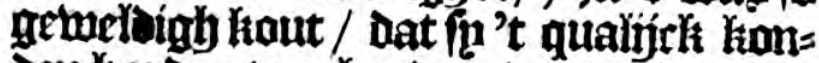
den barden te arbepden / maer den un= terften noot dede haer daer ín boogt ba: ren. Jen 30. mag de wint (B. ende (B). $\mathscr{Z}$ : 由. ende't hadie alle dien nacth pa ge/meent/alg' 't oock dede den gant/ichen Daugh / Dat pu geen bout mochten balen/ fo boogh land de fineeu ap maltsanderen. Sy maechten een gaet bier bn't bupes om de aerde t' ontongen / en die rontom bet hump in te ftoumen / op dat bet alfon te dirbter jýn foude / maer 't was ber = geefiche moente/ want de aerde lwas fon hatt ente diep bebzaten/Dat fue niet on: Dopen en koniden / of 't foube baer al te beel fouts geltaft bebifien / fon bat fu't moeften laten ftaen.

Ten theeden (1)tabrige recljten fu het fung ap / ende fetten daer boben en Mlep-boom ban bevzoren fineeth. Den

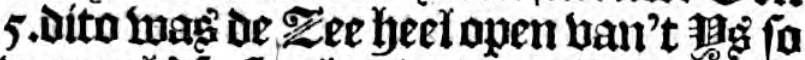
werte alge fie fien konde/maer lagen noch eben ftijf Geveren/ende't Schip fat wel twee of drie boet geboenet on 't 1 is / fon dat frr liet anier en konden bermertien of fir lagen tot de uront toe bebzoten/het wat daer bier de halbe ladoem diep. Dese

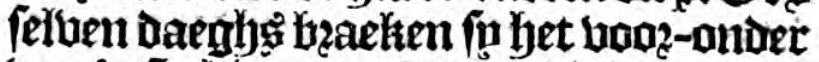
ban't Srtyp ap/enie met die delen bef:

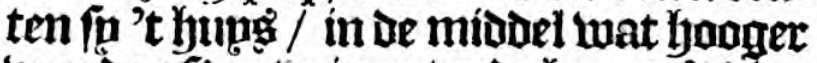
boos de af wateringe/ ende kregen't dien 
26 Derde Schip-vaert van Willem Barentfz.

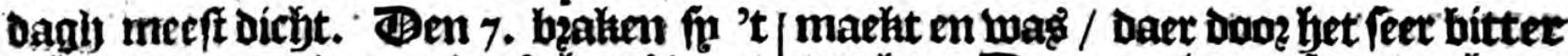

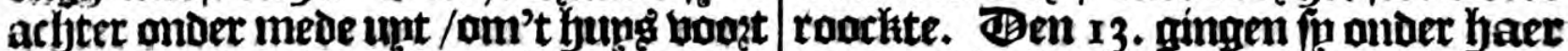

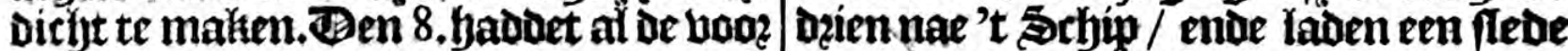

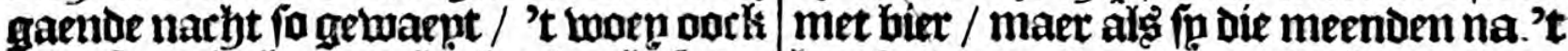
ende ineenjachte den lyelen dauth foge= weldigh / Dat bet fetyeen men ionde ge= fmanet lyebhen die in de locht gektamen hadoe / jae't waer eener niet mogelify ge = weeft allyadoer zijn lijf aengetangben/ een arhipg-lentoe boots te gaen/want't

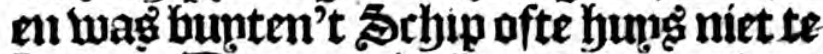
haxden. Ten 10. Dita begon't weer wat te heteren/alfon dat fa weder unt bet ba Schip beftonden te gaen/ende't gebeur= De Datter een wan't bolek unt het 'schip gaende cen $25 e e r$ in 't gemaet liep / dien

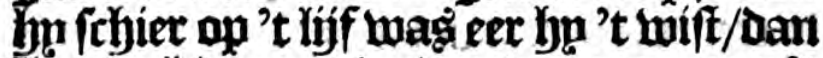
liep mel dapper weder te rugge nae 't Sochip/ende de 2 serr yem nae. In't na: foopen quam de 2 Beer ter plaet fé daer fó booz-heen den 2 Seer hadden geftelt te bebiefen / bie nu al onder de fnee betwelt was / maer alf́ dat sijn eene pont noch boben unt-ftack / Daer biteef defe $2 B e r e$ faen / ende dooz dit vertaeven quam de man hefyottden in't Schip/roepende feer

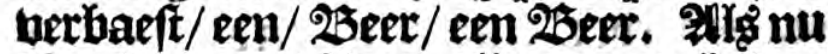
D' ander maetso boz zín raepen boben quamen om nae den 2 Beer te lehieten/fa en Konden fu unt baex oogen niet fien/ bermitas den bitteren rabe / die fu gedu= rende't quade weder in't sebip befioten zijnoe/geleden badien / ende niet te tijoen en foute gretweeft beliben om geen Gelt ter lwerelt / maer maeften bat noch

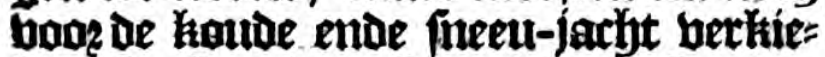
fen / fon fo ander baet leben behouden wilden. Be 2 Seer bertoef de mede niet Imuthe aldaer / maer packite hem fiuct. Den I r.bzachten fip baren wijnende an= Deten boograet aen lant/ ende Den 12 . be= gahen fo baer met de belft van't volck in't bupgs / Doen vaoz d' Rerffe mael daet in flapende/ maer leden feer gronte hou= oe / Dage Datter noch geen kope nemaekt en waren/ende fọ niet te beel deckfelła en hadDen/kmoen aock green bier boutden/ betmits de febou-fteen wach niet obe= hutgis te gaen flepen / ontfacker onver= fien fo getwetoigren wint/onweder ende houde/Dat ín haer wederom maefté in 't Brhip beqeben / vermits fo bet bunten niet haxben honben/ maeften daetom bet 2 Sier back bunten op de fiede laten leggen; ; anderen daeght bonden fa de Conme daer't in wa:/3inde een Ilopen= bat/aen den bodem in ftuchen gebzoren/ ende' $t$ 2Bier datter unt Yiep bzons fo baft aen den hodem tae / als of het met eenig baft boudende lím daet aen badoe ge: hecht gemeeft $/$ ig ftelden de tommen in't bupto op den bodem / ende dzontcken die booz eerftiedigh / maer moeften't Bier 'melten / want daer was naumelifer res nit onbebzoren nat in de ton/Dach in Dat Feluighe huas de ganticlye hracht ban't 25ier/alf dat bet beel te fterch was ont dzinckén / ende 't geene bevzoren wagt imarckte als water/ Daerom menuboen fin't als't tgefimolten was noner malkm: Deren/ maer 't was heel krachteloog ent de fimaecthelongs. Den 16. Dito hadder

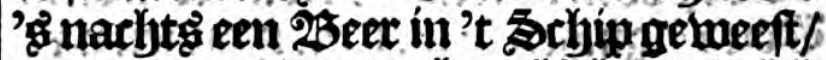
maer tegeng ben dagh / als ing 't bolck hoogde / was hp weder daer unt meloo: pen. Op den ielven tít bzaken fo de ka junt wegh / om de deelen te hefigenaen

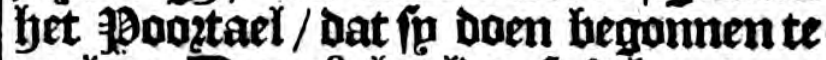
maken. Ben 18. haetden fín't bzont unt de fehunt/Die fo op't ilant gefleept bad= den / ald mede den wín / Die noch niet feet beberofen mas / hae wel ip wel om: trent fegi weeken daer gelegen hadoen / ende bet dickmaels feer hart gebooren hadde. Den 19. Dito watender niet dam twee man ende een jonten t' Scheep / ende daer quam een 2 beer bie met ne= welt willde in't Sechip wefen/ waer dong het bolch feer ber ftheicht waren/ende fa= gen elex om een gret heen famen / be twee fpzongen in 'trupm/ende de fante Glam in 't foclietwant : Allibiolertiont quamen 


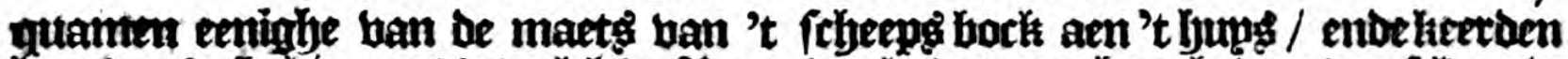
Gung na't Schip tae / 't welch ae $23 e e r$ den bodem om boogh / om den felben in

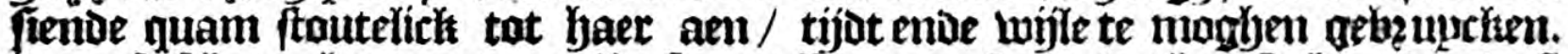

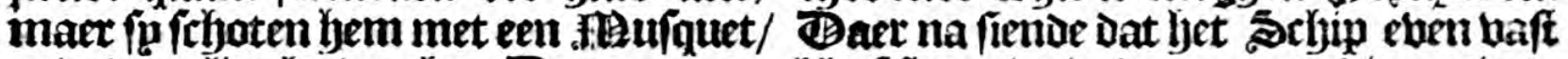
ende doen lieph hp wegh. Den 20. qua= bleef fitten/ ende datter geen ding minte men fy om't 2 Bier boozt unt bet Schip te berwachten was alş openinghe bain't Galen / ende bebonden dattex fommighe water / foo bzachten fin't werp-ancker Gaten in ftucken geveoren waren / ja be weder't fetbeep/op Dat bet onder de fuleen

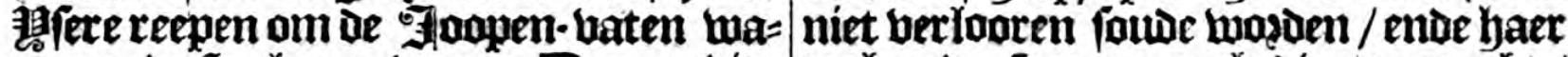
ren mede fturken grebzoren. Ben 24.Dito teghen de Samer noch Dienen macht /

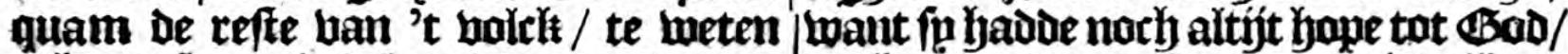

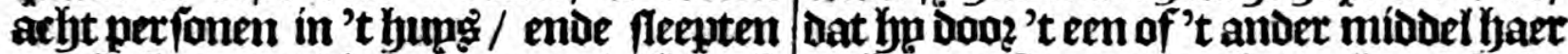
een fieck man / die feer teer ende f wack tegthen de somer nacly nae hupg faude was/op een fiede. Sn fieepten oock met betpen.

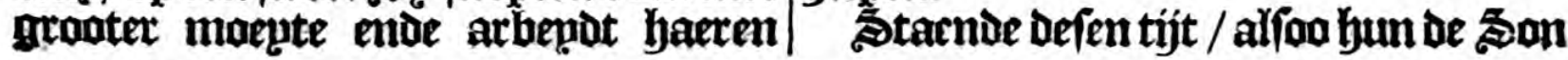
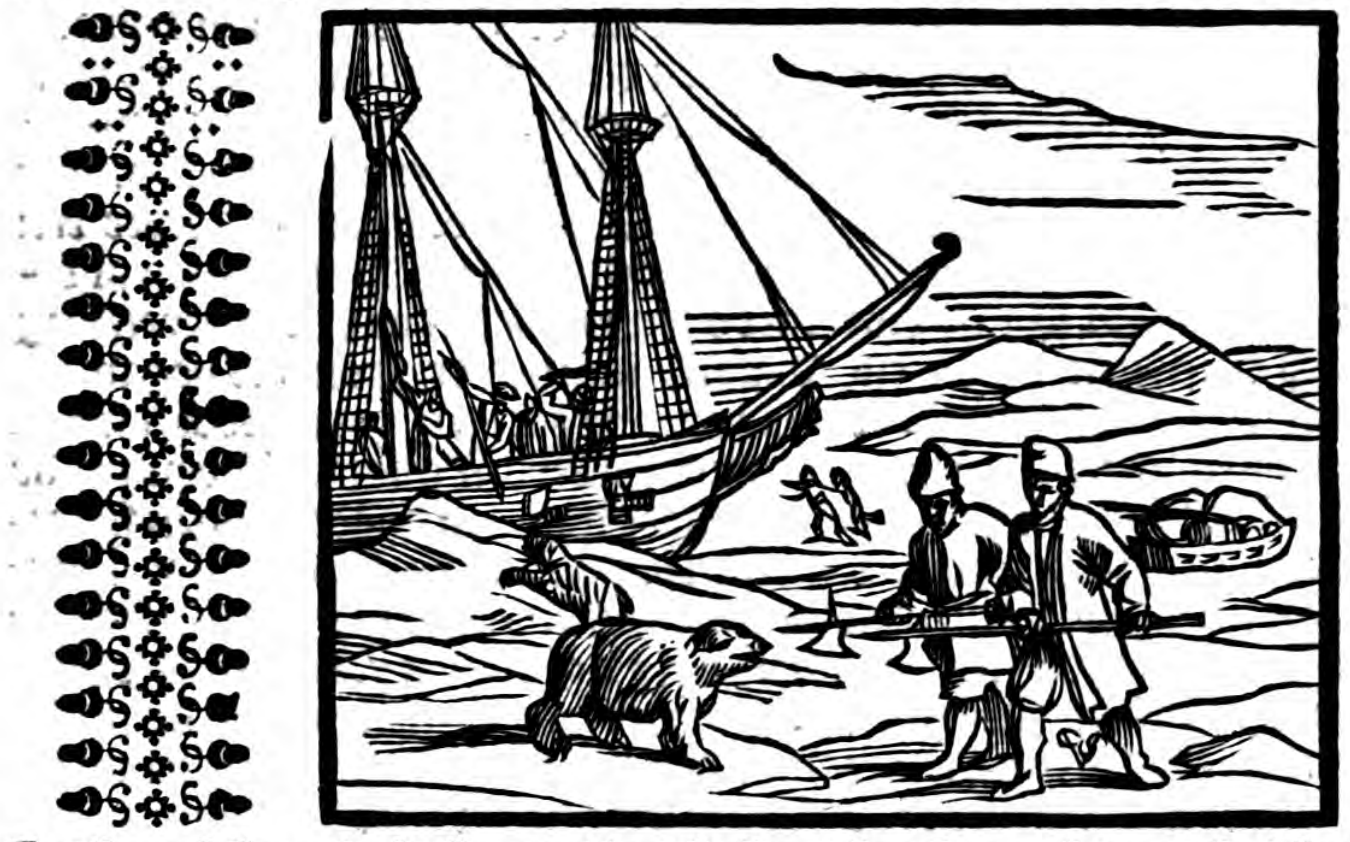

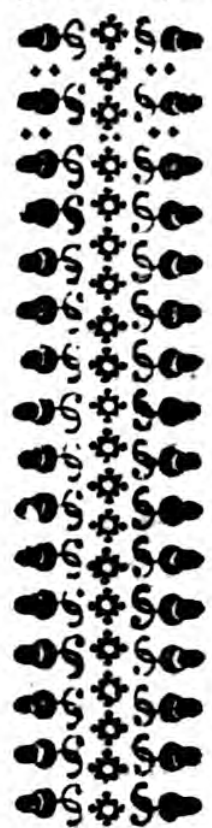

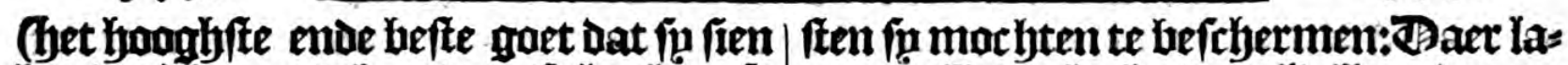

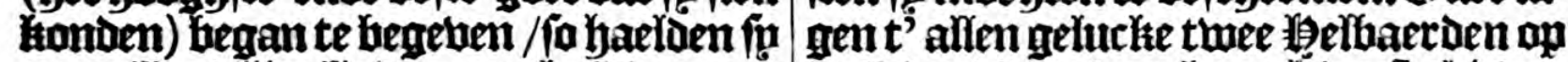

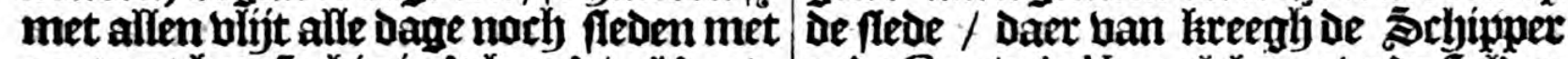

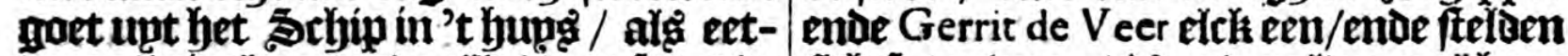

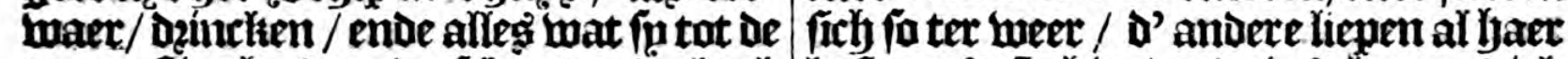
toe-cuftingthe bam de febuut ende back befte na't 'schip / ende in 't loopen viel=

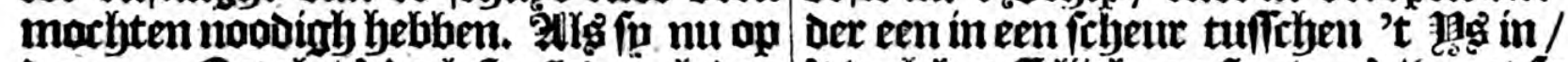

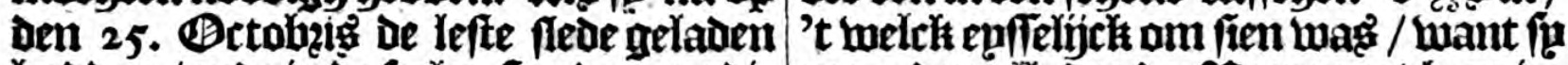

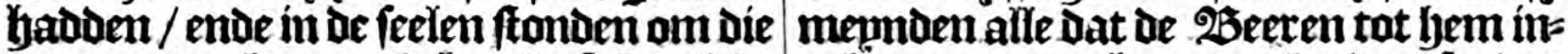
ftrijot. boast te trecken nae't bung / fo heecli de

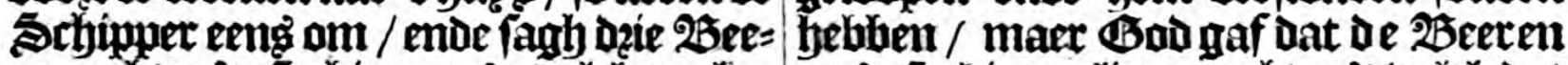

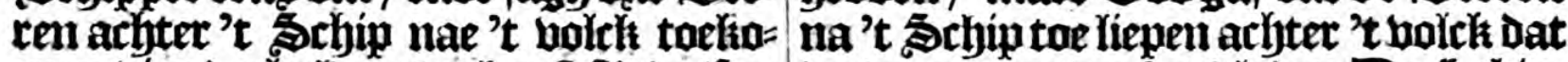

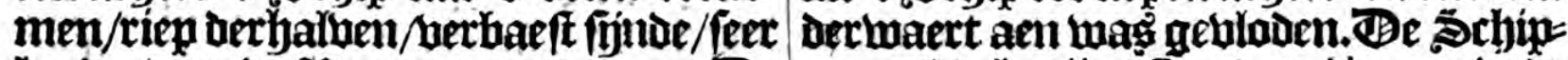
lupde / am de 2 Beren te vervaren: De per midoelertít / Gerrit de Veer,ende de

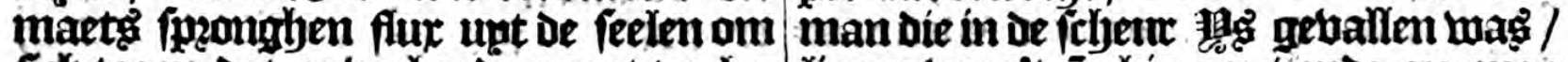

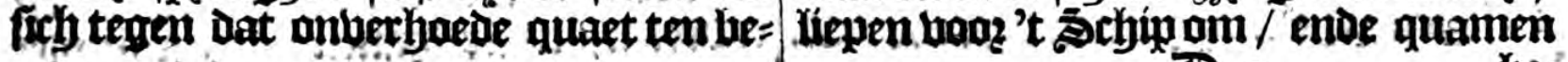




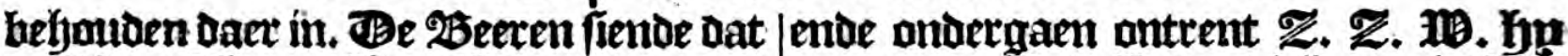

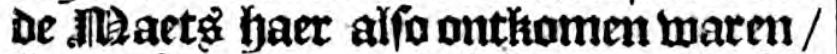
liepen feer weetmoedigh nae 't Sorhip toe / ende't bolch anders geen geheer bebbende dan de boonaembe twee $\$$ haerden / alfo fugaer niet genoenly daer op dozften berlaten / hielen de 2 Seeren baft gaende met werpen ban bzanotbouten ende anders / Daer fo t' elcltenge nae liepen arge en bonot na de fteen boet. Daer wert een Mal an gefthicht onder in de Cambunto om vuer te flaen / ende een ander bin sppieffen; uper en kanden pp

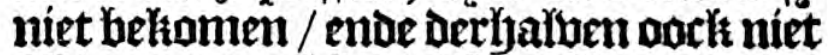
felyieten/oberfultro / alfo oe Beeren eben ftout aenquamen/fabebben pa deneenen met een 鼻elbaert reebt op de fnupt ge= woupen/ daer doos hp hem feluen getreft bindende/ allenedtengs af werch / ende de ander twee / Die fo groot niet en waren / tringen mede ban langer bant wegb: De inamen danckten Bodt dat fo alfo van Die felle . Mltanftetg ont/lagen waren/en= de fieepten de fiede met berughode nae't bups tae. Den 26. Dito fagen pp beel open water bicht bu 't ilamot / mact 't 1 贃

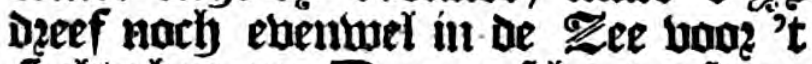
Echin heenen. Den 27. Fljooten f́pen witte $100 \mathrm{~s}$ / die fu aten / enve maeckte. dhebzaden zínde feer ats de ltonínen Doen. Ten fetben dag ftelden fy onk lyaer (Bozlonie weder dat de ktock floeg / ende maechten een lamp toe om 's nachts voer te bzanden / Daer toe fin gebzupch= ten het sefmolten bet bam den 2 Beer. Den 29 baelden fo cingel bam de frant met fleaen / en ftropsen bet aber't zepl bat ober 't hupg' law/op dat bet fo beel te dichter ende warmer we fen foude/want de deelen lagen ongedzeben ap 't hups? alfo fo Doos't quaet weder belet waren die te dzýluen.

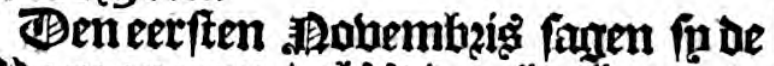
Iflaen oxgaen / ats't boncker begon te wazuen/in't (Oaften/ende de son ging

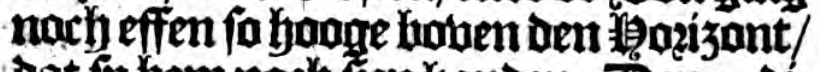
dat fo fem noch ien tanden. Jen 2, Di=

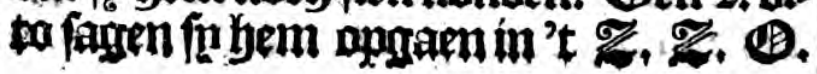
quam met injn balle rondinfept niet bo:

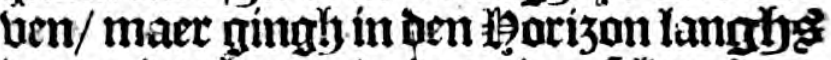
der aetuen fenen/ente op den feluen bay werter een Bogs met een híjl getwazuen / die fu bieden ende aten. Den 3. gingth

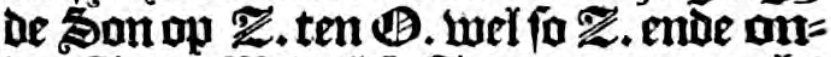
cer $\mathscr{Z}$. ten $\mathfrak{n}$. wel fo $\mathscr{Z}$.ende men marbt Doen in Den de Son maer effen /ien/nochtang wa't Ulanot daex ju bem op peptoen wel fou boog alg de marg van baer srbip. Ben 4. al waft ftil weder/ fo en faren fo nocts= tan' De Some niet meer / want by lan= ger niet boben denlyarizan quam. Doen dzonmert de de Ch hiruxgín een bas om te ftoben ban een 1 Bijn-pijp/vaer wingen fot d'een bong d' ander na altemet ím/en= be bonten fich daer gantifh wel bp. Bp den felben dauh binghen in weder een witte $100 \mathrm{~g} /$ welelt genierte fich nu al temet openbaerde/terlwíl de Seeren weg waren / Die met be son ong benaben/ enoe niet weder en quamen boog dat oolt de son wederom quam. Toen mu de Son baet vetiaten badoe / faren fu we: derom dang ende nacht de Jilaen pon= Der ondergaen / alfo po in baer boogbfte tepchen yimgh. Den 7. dita wag' 't dux= fter weder / ende men fande de nabht ban ben daugh qualífelt ondertenmen / fonverling om dat baer ftaen badoe / Daer boos fo bermoeden geen dauth aly' 't alreede dagh was: : Sp hadoen bien nacht unt de kap niet ge: weeft dam um baer water te maecliten / daterom wiften fon niet wel of 't light bat

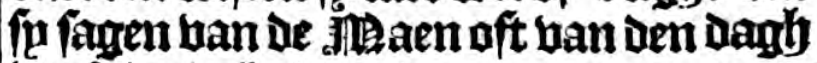
wag / ende badoen daer ban onder mal= famberen beel bifpunten / maer alts't al om quam / fo wat 't wuel op't hooghife ban ben Daub. Den 8. Deeloen in' 't bzoot moer malkanderen am / ende elch man freer bier pont ende thien loat in de acht dagen/Daer fo booz beenen maer biff of = te jeg daghen mese toequamen / bleefch enve bifch lwag haer noch geen noot om te deelen / maet dzanch badoen fo nict 


\section{Om't Noorden na China.}

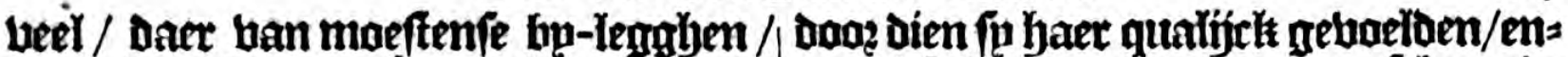

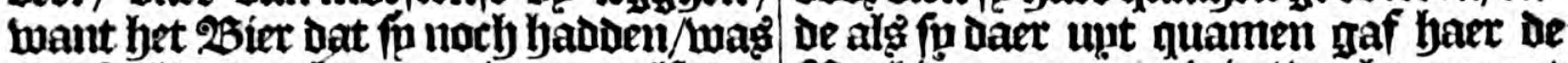
meeft al de Frarbt unt gebzoren/alfo dat 2Barbier en purgatie in/Dat baer groot

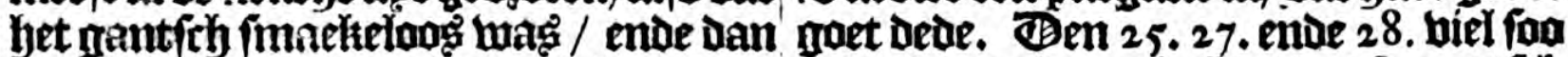

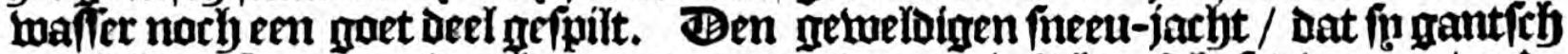

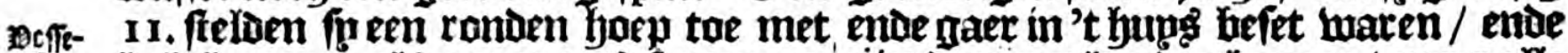
bal. habet-garen als een net $t$ ' famen ge: niet Daer upt thonden homen / maer alle bzept om zoffente bangen foo dat men baer dingen bimeng humg moeften doen

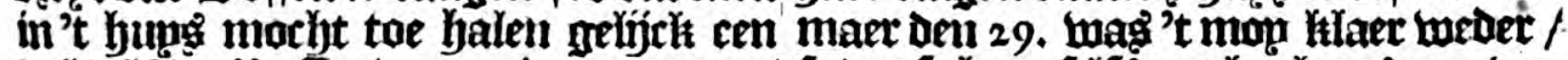

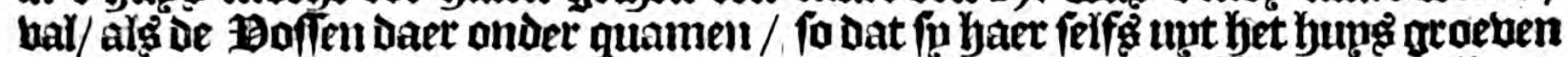

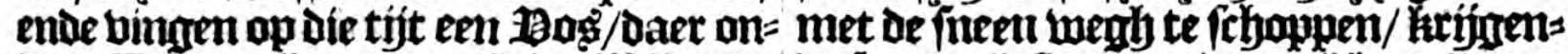

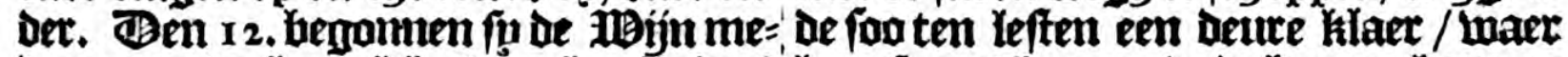

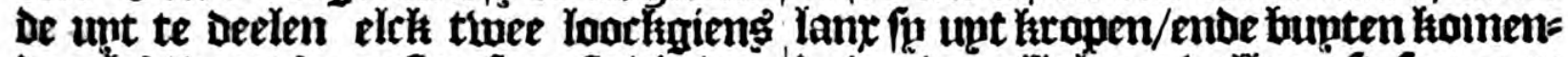

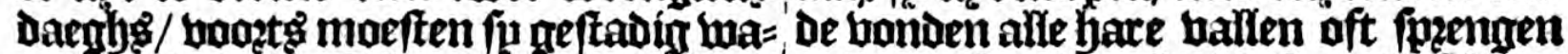

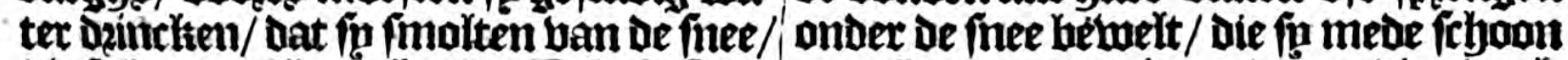

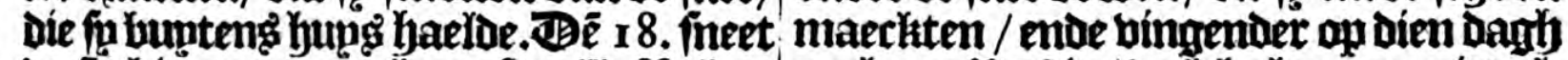

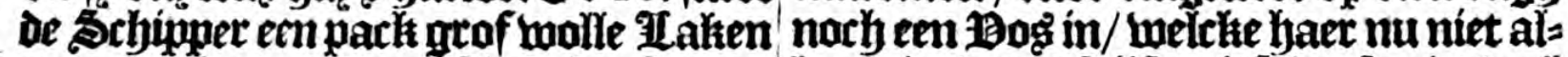

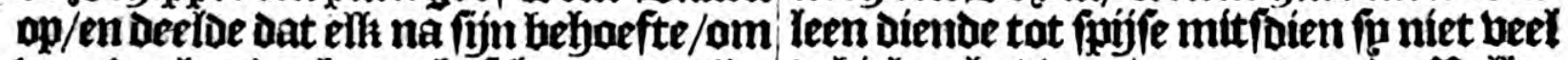

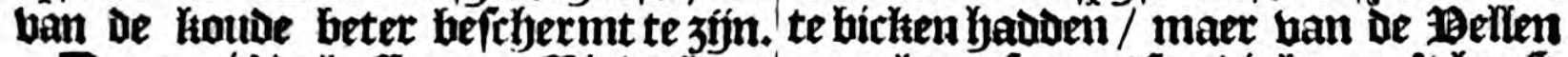

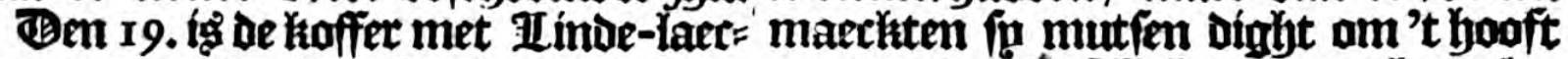
ken geapent / ende onder be maet unt= toe / om ban befe felle hontoe wat beter bes medeelt tot Ysembien/ want be tijt begaf frhermt te zim.

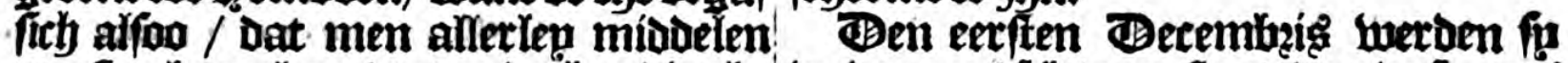

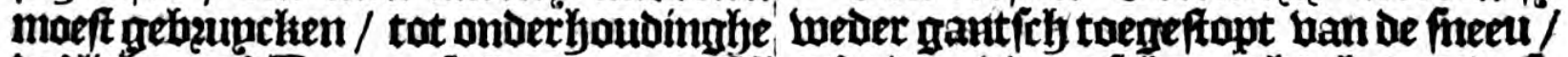

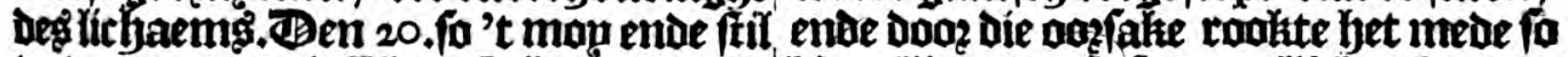

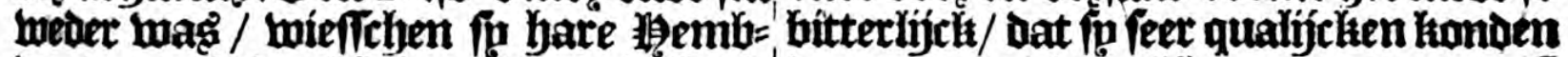

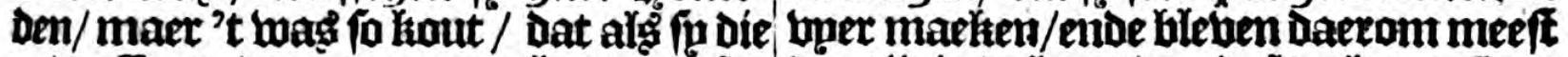

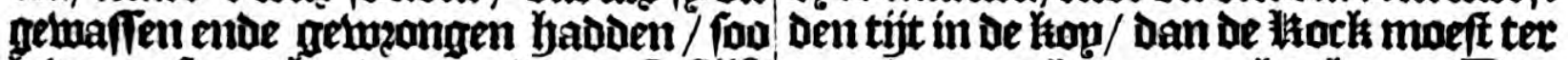
bebrocenfe unt bet warme water fo ftijf/, noot uger makten om te hoolken. Bon

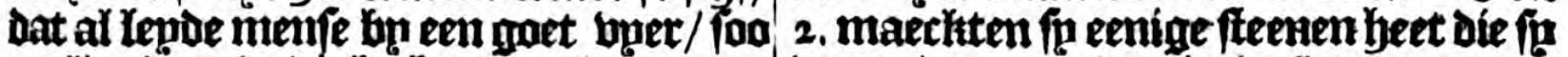
ontliet de 3 pbe die by het buer lay / maet ben anderen gaven in de konen om de

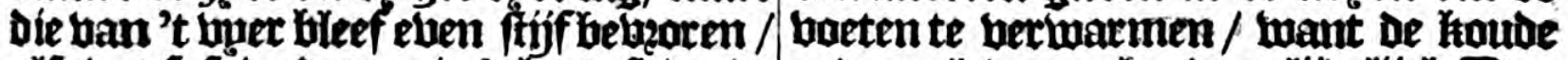

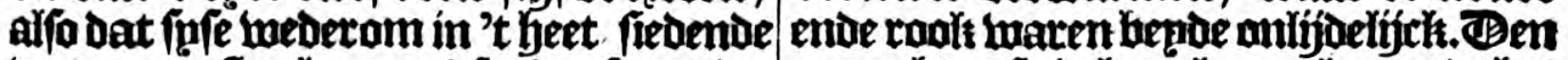
twater maeften lemgen / foudenfe ontons 3 . mochten fu in hare kopen leggende het

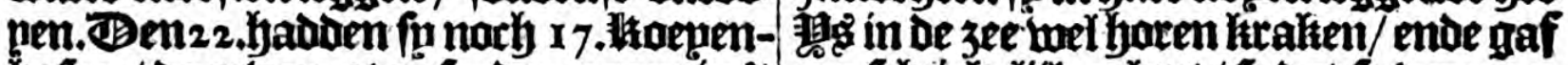

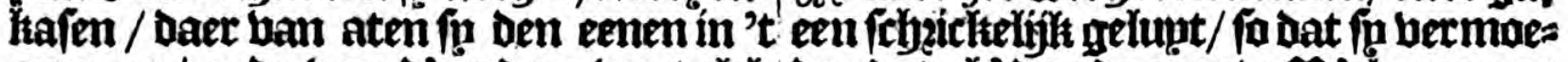

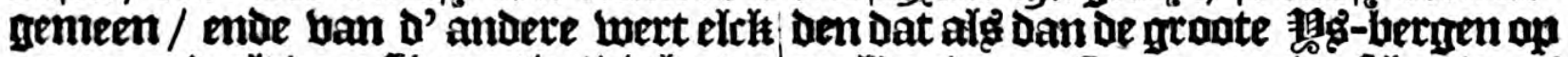

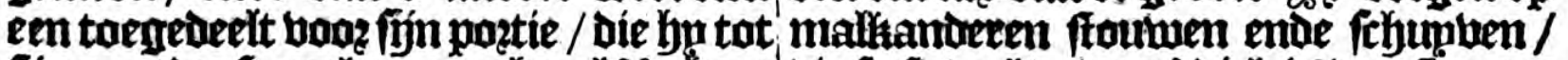

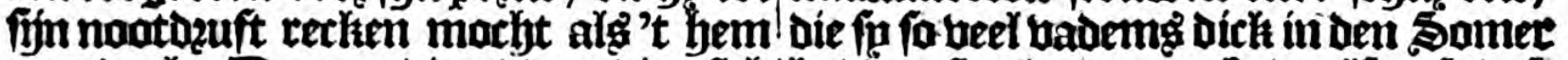

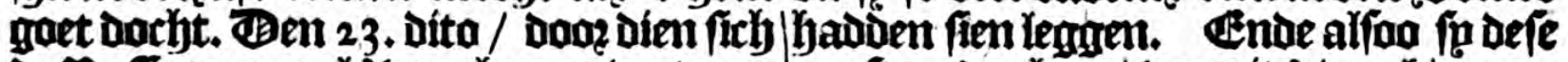

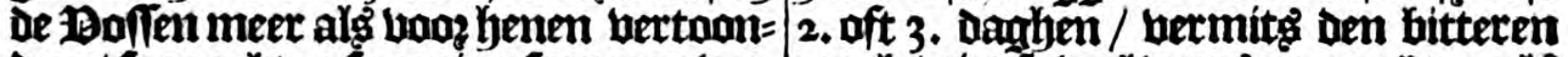

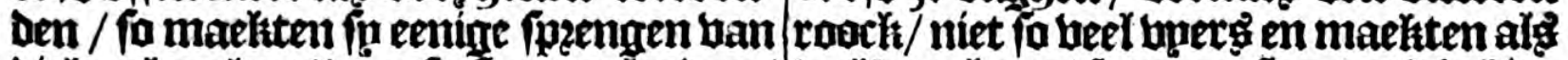

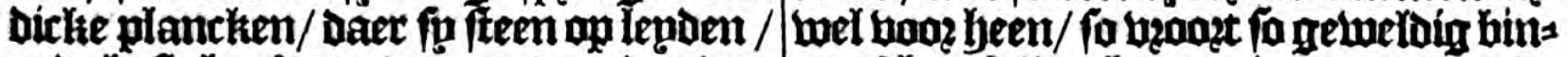
ende beftaken't rontom met eunden ban nen hupgs / Dat het aen be manden ende

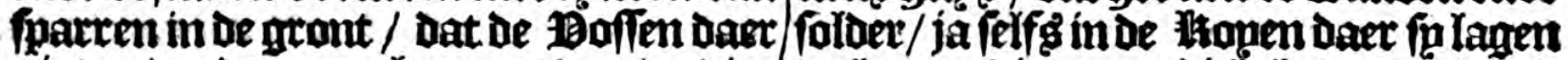
niet ander Doog mochten graben/en vin= wet twee bingeren bick bebzoten wag.

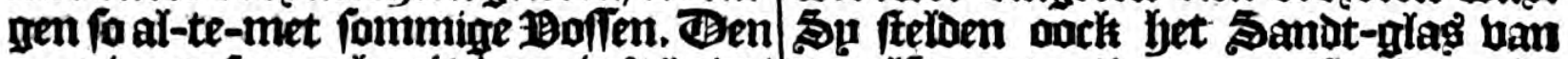
24. gingen fin met baet bieten in't baot/ thaelf uren toe/daer op geftadig waxtht 
30 Derde Schip-vaert van Willem Barentfz.

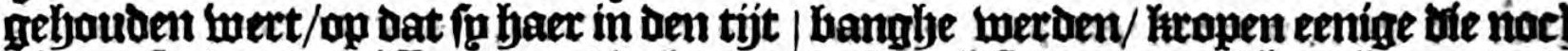
niet en fouden bergiffen / want de koude de hiloech/te waten ugh haer hopen/end

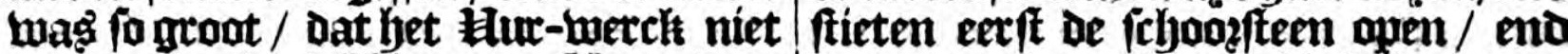
gaen en knonde / al bingmen feljoon daer daer na de deur / maer die de deux ope

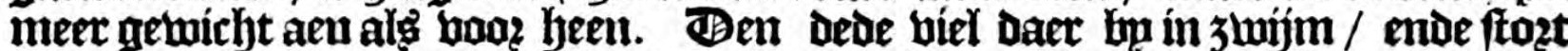
* elle 6. Dito wag' 't poo geweldigh Kout / Dat houde. De maets malfanoeren Deerfick aen= fagen/ beer fende fo' 't alfa boostaen nocly funder fontde wozde/dat pu ban de taude fouden bebben moeten vergaen / want wat buer dat fu maeckten fo kanown fin haer evenwel niet betwarmen / jae de Seréflye Seclite (die doch po beet ít) be= b2002 gant /ef feer/fon dat menfe ober't bper inoft imelten : ald 't deel-bag was/ enie fo om den tweeden baubj elck om= trent een half pintjen daer ban tot baex Deel hregen/ daer mede fig haer fon lange moeften onderbouden/oft anders water Drinclten/ Dat immer met de houlde niet wel ober een en quam/ ende niet en be: baef de met fineeulu gehoelt te wozben / want die mafier be moeder ban.

Den 7. wat $t$ nocb eben quaet weder/ จn regs ende alfa pu onderlinge raetfaegden bae aen ban in 't beft aenleggen fouden om de knude feen / bat te wederfaen / po wafler een die gaf baer be boo/ datmen de fteen-kaolen/Die fp me= nae't hoft. de gebacht badoen upt het Scljip/miter unterfiter noot gelozunchen poude / en baet buet bam maken/alfo't een freeten bzant was / ende lange duet be : Opp den abont lepion fo een goet buet baer ban aen / Dat quoote hitte gaf / maer fu bad= oen fich niet gewacht boo? de weer ftupt/ want obermitg de warmte lyark fo wel bernuichte / bonden in (om die lange te Jouben) raedtaem alle de deuten ende De feboosfteen diclyt toe te fiopuen / ende gingben alfocelcx na fín fope om te flapen / wel gemoet zúnde doog de berkire = gen warmte/ende lanobe tíjot met mal= handeren pzatende: Jillaet in 't epnoe bebingh haer (boch den eenen meer alg ben amoeren) een groote dumfétingbe/ 't weich pu eerft gewaer werden boos een Die fiek was / ende daetom fulcr te min betozagben mocht. Fulg fi mu alle feer op't (neeu neder/'t welch boarende Ger ric de Veer, alge met zín knop naeft aen o beut legrenise lyaelde fuct edicl / eno weef hem die in fijn aen/icht/ foo dat h nocly bequam. Te beuren glyeopen zijnde / merden fu alle wederom dons o houde berquickt / die haer te booren fo barden bpant geweeft badoe/ fouden at bets ongetwiffelt alle als in een 3 wijir glyeftoguen bebben. Taer nae gaf hae

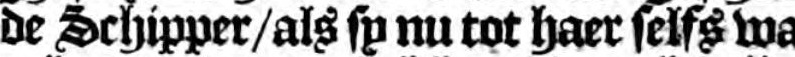
gétomen waren / elch een Dzonch inín om't baxt wat te fterctten. Den 9.10.e I I. wa't heloer fklaer wever / enve o locht bol fterten / maer fo onlíjolific kout/ Dat het ben muerfocliten qualize gelooben foude/want de fichonen bebza ren 't bolck foo hart aldeen fooren om de boeten / foo dat fp niet lamer eenige fchaenen honde gebountem/maer maelt ten wijoen hlompen / bet bobenfte bar fehapen bellen baer fin met dace of te bie paer focken ober malfanoeren metroc Een mochten in gaen/om alfa de boeter te bertwarmen / want felf g be hleedere op baer lijf waren ban den bogft wit unt gheflandjen ende berípt/ende polyaeft wat lange buyten' render blaren ende bumlen aen bae aenficht ende ooren. Den 14 . dito name fa de booglyte ban de rechte feljouder ba

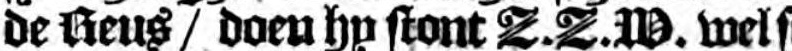
weftelijel / ende bebonden bes poul hooglyte 76. graden. Den 18 , gingthe iu met haer feben mannen nae' 't schi oim te fien bae het daet geftelt was/ eno bebonden bat het water in 18. Danbe (Dat in daer niet ofjelweeft waren) $\mathrm{ee}$ bupm gewafien was / boe wel bet gee

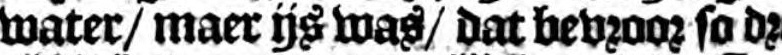
ald 't boben quam/gelích pock be (I) laiten die fo met water upt ollamt mede genomen hadoen tot de ncont to hebzoren 


\section{Om't Noorden na China.}

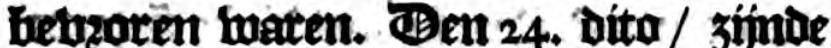
Herti-abonot / moeben ip haer unt het bung/'t wellk nu meeft alle bage te boen wag / enve faghen beel opien water in jee/want fip badoen't ýgs genoeg booren fratten / ende boe wel datter meen dag

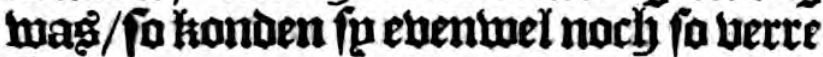
fien.

Bp 1 ters-dand was't noch al quaet

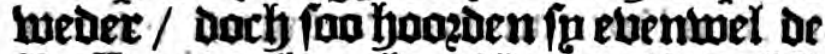
TBofen ober haer bupgi loouen/'t melek fommitre fepoen een quaet tepclien te wefen/ende ats men beargue baerom / fou berter geantwoost/ om dat menfe niet in te pot of t aen het Spit en mocht felten/ want bat badoe dan een moedt tepclien getweeft. De houte was alle $\mathrm{Da}=$ gen fo groot/Dat het uper nau bitte gaf/ want als fy baer woeten nae't bper fta: hen / foo berbzanden fp eer haer kouffeu dan fu de waxmte ueboelden / foo dat fo ftadinty gevioeg te doen haoden met lap: pen / fae baoden fip't niet eet gerooclten alf geboelt / foudenfe eer gantich ber: bzanot hebiben / Dan fu't getwaet getwoz= Den haoden.

Tha dat fu nu aloug met urooter houde/ períchel ende ongemach lyet jaer ten

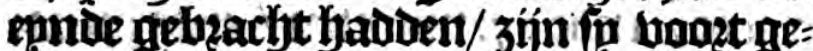
treben't jaer nae de geboozte onfes

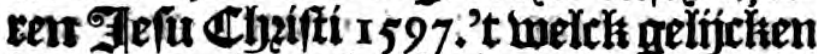
ingangh gebaut beeft met den untgang deg jaers bookleden/mant fet meer bleef then quaret ende hout. Sp hegomnen feer op de felbe tífot haeren dibín bu klepne maetfteng unt te deelen om de twee da:

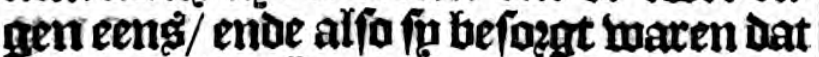
bet noch langlye aeniloopen foube eer ip ban daer fouden raecken (waer toe fom: tífot gant/ch Klepmen maet hadoen) fo pareden de fommigbe noch den felven Jibín fo langh aldi p mochten / om tex noot altifot mat in boozraet te bebben. Den 4. Jlanuarí ftaectien in (am te we= ten boe de wint was) en halbe lancie boben unt de Schoorfeen met een Hilewn Doexten oft't bleugeltjen daet aen/maer maeften fract Daer nae fien boe't upt= waepde/want fo dra alt't unt/tal / was't io finf bebzoren alderuhoubt / en konde danniet dzaepen nocly uptwapen (Ben 5. bet weer wat befadigt jínde/ groeben ip haete deur weder open/ na dat pp muettes lífte daugen na mailkander hadoen befloté aelweeftende niet een hooft monten unt= fteechen : Sog maechten ooclis alle dingly wederam klaer / faeloen bout in / ende thloof bent / waer met pp den gant fohen dagly doende waeren/om immers fo beet in voorcaet te hebben al's't Doenifít luas? beefende of fowederom machten alfoo beflooten wozoen. Filg fin nu den gants feljen dangh dug gefionft hadoen/ werden fo gedencliende dat bet dzie Honinghen juont was / ente begeeroen aen ben Brhipper/ dat fu tufiche al haer verdziet fich eeng wat vermaeften mochten/Daer toe by-brenghende den $\mathrm{H}_{\text {Hín }}$ die fo ber: paert ende in boozraet badde/ende Bams. ne-foecken met (1) In backende han om: trent twee pont meelg / dat fu mede ges numen yadoen om de katooefen te pau=pen/waer met fón fo bolích waren ald of

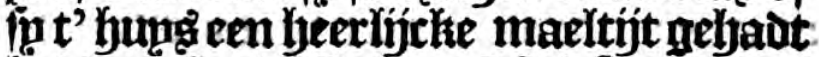
badoen: :sp deetien oock bief keng unt/ ende de Conftapel wag Coningth ban Nova Sembla, een ILmot dat wer twee

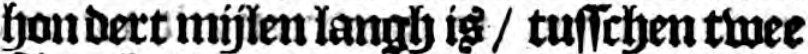
Zeen befioten.

Den ro. Ilamuaxí bevonden pa bat lyet water in't Selyip wel een boet ge: mafien wag. Den i 2.namen fu be hoogs te van Oculus Tauri, een welthekenden fterre/ ende bebonden dat defe metinghe met die ban eenige andere fterre/atso of ban be Sonne wel ober een quam / ende dat fu aldaer waeren op be hoonthte ban. 76.graden/eer hooger dan laeger. Den 13. wat 't kilaer ende ftil weder/ ende fi konden boen merelten bat het licht ban. Jen dagh began te meerberen / want ip fiepen unt en fithoten De hloot/Die fu boos heen niet hadoen ftomen fien loopen. Dan baen boogt rimuen fin altemet bup: ten fougs / om laaere leden wat te ber: ftecclen metgaen/ werpen/. ende loos 
pen / u bat fn niet berlammen fouben/ ende begonnen eenighe roadiptsendt aen be lucht te fien/als een fehijniel of t booz= bode ban de toekomenue Son: Daex quam oof wat meer warmte ober Dar/ foo batter/ algo fo een goet uper aen had ben/altemet ban de wanden ende folder ban't bumg / groote ftuctien ugi bielen / ende bet in hare kopen ontliet / bat boo? been niet ubefchiet en wags / hoe mante bueren dat fp pock maechten / voch des nacht boogt wederom euen haxt. Den 18. Dito / bermitg haer beantfout baft begoun te minieren/lenden fi weser fteen-ltoolen aen/maer ponver de felyoazfieen te foppen / Daer en boeren fo niet qualífect mede / maer achteden 't eben= wel beter de foolen te homiden/ende het bout wat paeclích her aen te leggen/ om bat de hoolen baer ín toefomenoen tijt / als fig met de open fehupten naer bups: forwen baxen / meex dienftelích handen zín. IEnde aifo't beot al-te-met began te frimpen / boos bat fommighe tonnen baer boile wigt niet en badoen/fo moeft= men be untweelinge bock wat bermindes ren/ ende die te booren met paren wat ober gehoujen badoen / mochten 't nu Daer wet ímboctien. Het Bofien ban= gen begon oack te minderen/'t meick een bookbode was dat de 3 seeren haeft fouben weder famen / fo p pamaels ge $=$ waer werien.

moevers Den 24. Fanuarí wag't mou fylaet hounft tweder / ende la cob van Heemskerck met Don/ die Gerrit de Veer ende nacly een derde zín in mu in

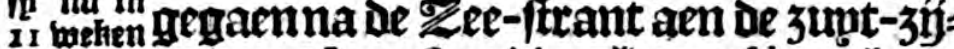
enve 5 das de ban Nova Sembla, allwaet Veer, but: nen niet ten lyaer aller niffinge / alleteerft fauth de baober. Him ban der Somne / Daer ober ip fiuex wederam naet hupg paeden/om't felve Willem Barentfz ende d' andere maet boo een blijoe trífoingtie te berkandigen: Willem Barentiz atgen filoet enve ex= Laren Stumman / wilbe Dat geen/mg gelooben als omtrent noch 14 , dauben boog ien tifot zínde dat fich be somne baet on die booghte ouenbaren foutbe:

\section{Willem Barentfz.}

Daer tegen ftreten finlyact bie de som gefien badien/ende biet duer gefthieder ber f́lbepien treddingen. Den 25. ent 26. wag't miftigh weber/fo dat men nie fien lionde / ende hietom meenden de git ne die contraxie getwet havoen / Dat fu newounen hadoe/maer op Den 27 . Kilaen De't weer op/ende boen fagen pal te fi meu de son in fín bolfe ronbigbept br

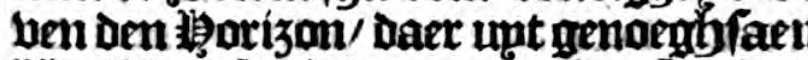
bilectl dat pip die den 24. oock gefien bai Den. Intaer alfa hier tegen wozt boozg went / dat dit frijot teghen't ueboele ban alle oude ende nieute seribenten jae tegen seloop der nathere / ende fo

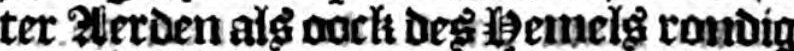
bent/Daetom fommige fegren / bat ber mitg daer in lange geen dagd geweef hadoe/De maets fich moeften berfiapen. ende quade gifingtye gemarckt bebien / fo bebien f $\mathrm{p} /$ op dat niemant haer en be denclie als of fu daer aen twífieloen/ in:

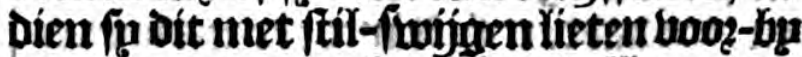
gaen/gaerne reechen/chap willen geben ban bit haer fegoljen/ om alfonte behif= in dat pin inaere metinge ende reeches ningle baft gaen. Toe som boe ph bem eerfit fandyen was in Aquario in ben 5. maect / ende 25. minuten / ende bu be longde bettoeft te bebben / nae baet eer fte giffinge/ tot Den 16 . geat/ ende 27 minuten/eer fy lyum aldaer op De foogt ban 76. grawen pube bexf hinen.

Den boor 13 26. Daut Flanuarí werd de fieclie man die fo onder haer gefet khap badoen ljeel 3 wark / ende geboeld bem feer qualijeft / want Iyg badide eet lant ende furur leger qehad : Sp bettroo ften ben! naer vermogen/met wat goet boos te fegaten/ ento ha ftier feen wepni nae mídoernacht, Jen 27. maeven fis

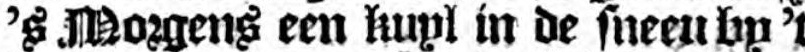
huph maer't wag's getweldig hout/ bat ig niet langhe bupteil en mochten bas: den/ende malftanderen t' elchenga moe:

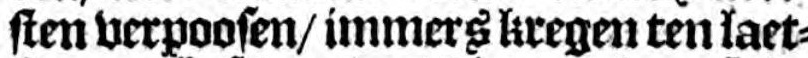
ften noch feben baet Diepte / Daet fp de Doode man in begraben mochten: Daex 


\section{Om't Noorden na China.}

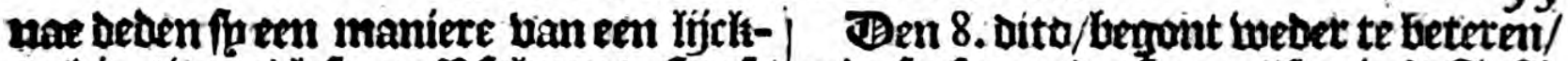

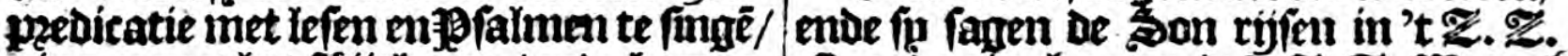

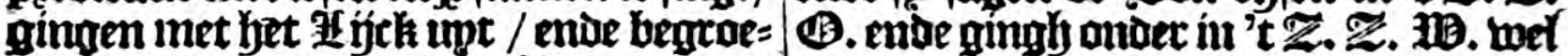

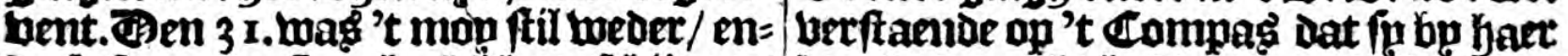

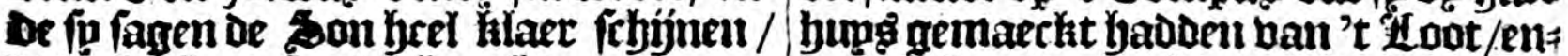
't welch yaet feet berbeugljoe.

Den 1. 2. 3. 4. 5. 6. ende 7. Tebzuarij wag' 't loogrgaeng quaet ongef tupmigh meder / baer booz haer be moet al te niet weder war begaf.

de geftelt op denrecthten atierioiaen al

Daer / anders ftheeloent op haer gemeene Compafien thee freket rumm.

Den 12. flebouarí waft klaer weder ende ftíl / De wint $\mathscr{F}$. Boen beben wag
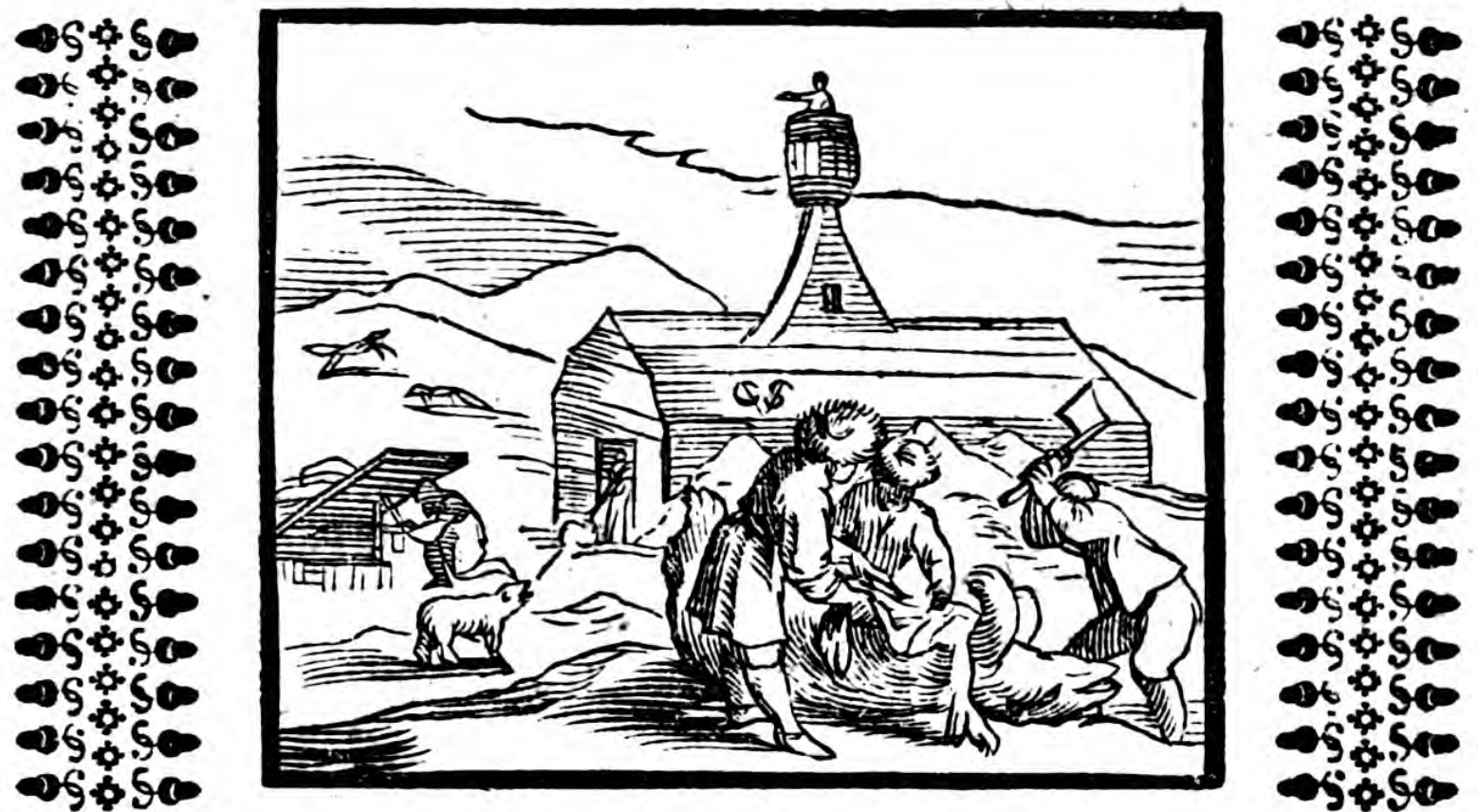

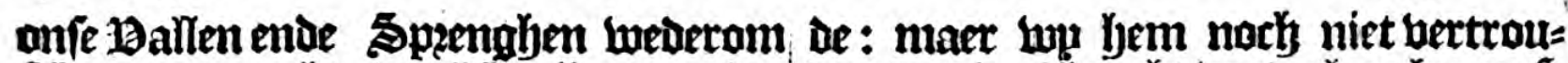

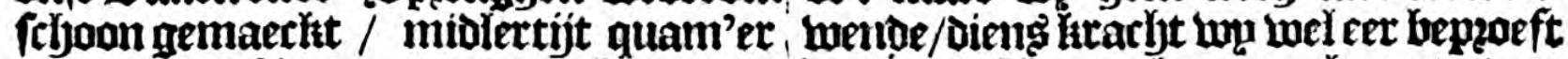
een grooten 2Seer tot ong na't hunges toe/ badien / feboten bem noch met twee

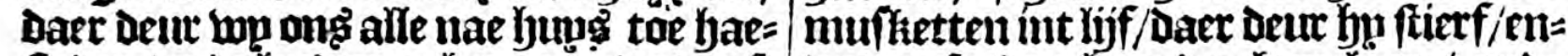

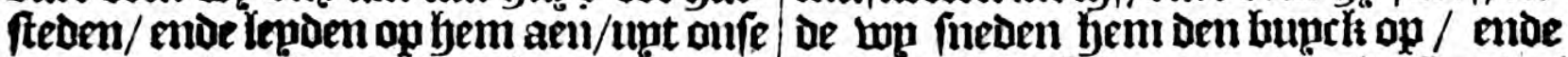

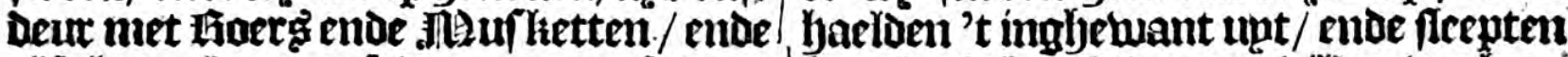
alfólgn rectjt op onfe deur quam/ fo wert

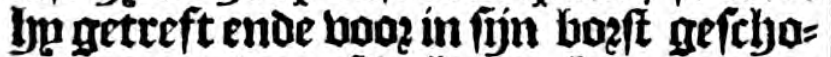
ten dattet Detur fijn bert arfiter aen den ftaert unt quam/oewt gant / alfo Dattet loodt fo plat was als een tao= pere dunt die men inet bamers plat flaet. Te 2Benz dit baelende / dede nocty ecn dauyeren fprong actjter unt / en liep

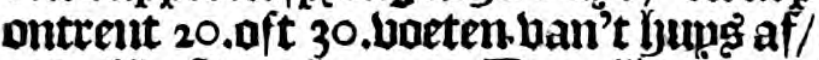
ende bleef daet leggen. Doen liepen wy alle flucr ten ljupje upt na den 23 epz toe/ ende bandent liem noch lebendicly/dat hp fún Jjooft noch na ong tae ap-beurde/als oft bu fien wiloe wie 't bem gedaen ljad= hem bu 't hupg/ daer na vilden we beni mide baelden daer wel bondert pout fimete upt / dat wu fmolten enve in de

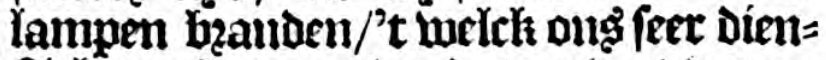

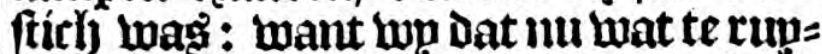
mer aentafteden / elide branden de lam= pen Doen nachten ober / Dat Lup te bogen Deur gebzeck ban't fimout niet morbiten Doen / ja elck in fiju Kon fuzande tot fijn

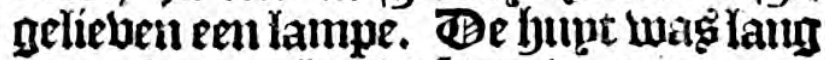
negen Laeten/Juged feven yoeten.

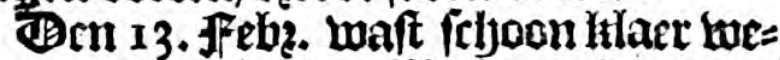

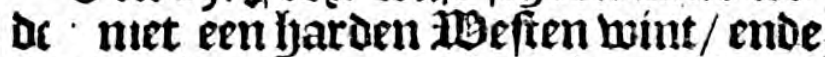

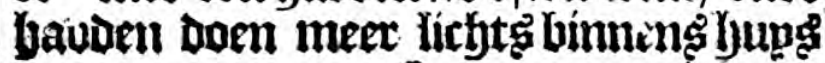


Derde Schip-yaert v beux 't banbei ban be lampen / dat ing altemet met fefen enbe atuersioen trifot

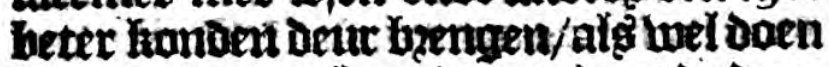
toe un den naxht ende darg dewa be buy.

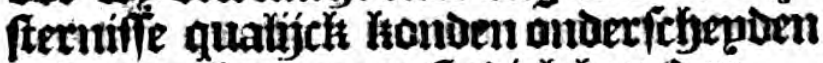
moe treen tampen geftadich beation.

Den I4. J'enguary waft hlaer weiser/ met een berowen foeften wint baos oen midoach: maer nae te midoach waft

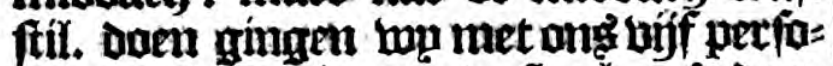
nen na 't '̧rihip om te fien boe 't Daet meftelt was / enoe bebonden dattet wa= ter in't Sclyip wermeerdert was / bach niet beel.

Den 15 . Fetbuarij waft quaet wedex/ ende twaende een bliegenden fta?m upten

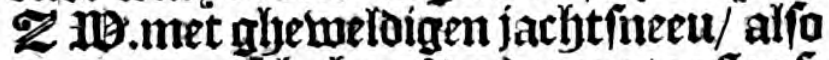
dattet gant iche hayg laederam toe ftoof.

Fnoer nacht quamen de Doffen na

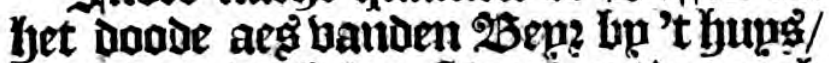
Daet detur bun hetweeft werden/ dat ooek alle de Sengen die daer ontrent waxen na ong toe fouden ftomen/ alfo dat wo raetfaem bonden dat wy bet aega diep onder 't freen fouden begraluen/ foo Dzae

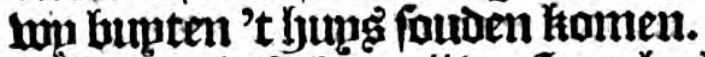

TBen 16. Jebzuarí waft norly al effen quaet weorr / ende jacht-íneetue met

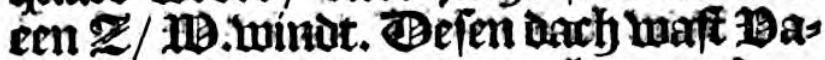
tetabont / enoe wo vermaeften ong een wennich in onfe deuck ende fimarictyent

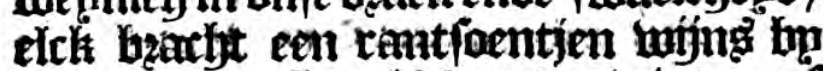
tot een gedaribtenis dat de winter af nam/enbe be vzolijitte tiót naltenter was.

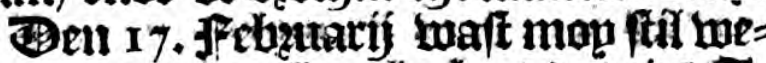
Der met een bankkere Yacht / be bint 2 . - Boen maefiten bu onfe bent heeberom

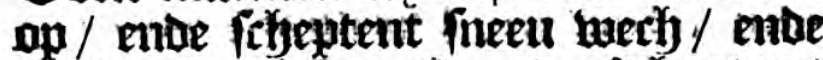
fimetent aess in't gat baer wan 't hout ugt alyelfacti baoben / ende beftoptent aner miver / om te oogfarect weth re atemen/

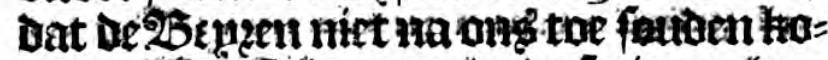

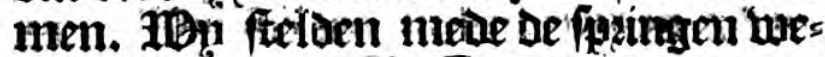
betam tae/om be Dorfen te bangen.

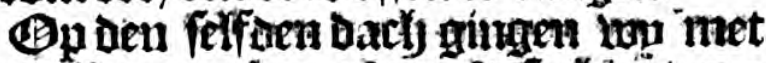

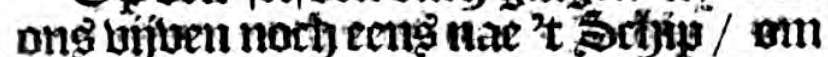

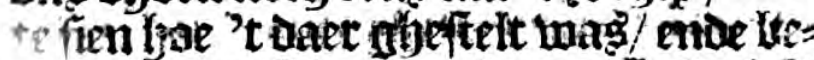

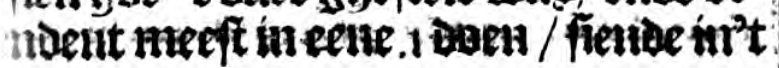

\section{Willem Barentfz.}

Schip beel boet-ftappen ban sepen/

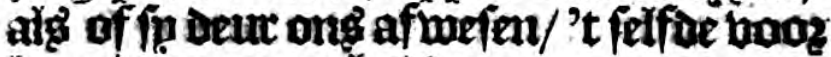
baex migenonien badoen.

Den 18, waft quart weder meteen Z.219. wint enve beel jactht-fitew/ende troote koube. Finder nacht alfoo wa SLampen beanoen: nive foumighe laet

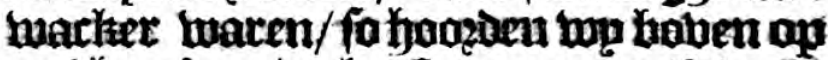
ong bruxg ecnige treefen trappen/'twelk deur 't ineeu meerier. febeen alft wel

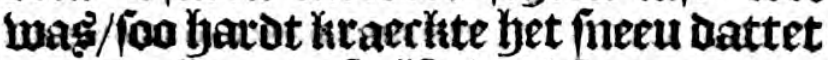
groot gelunt gaf alfa dat wu meenian dattet 25 epren lwaten maer alf dach tretwozden lwag / fon bondemen anders tren boet-ftappen dan wan bofen/ Dat wo meenden dat 25 eneen waren ghte= weeft : want de nacht die eenfarm ende

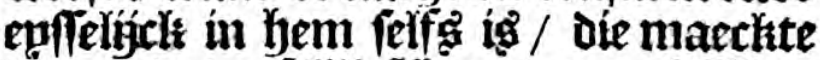
't gene dat eufelijht febeen/nog enfelijker.

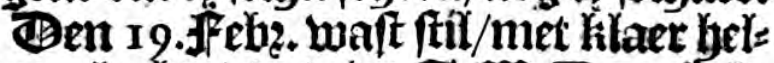

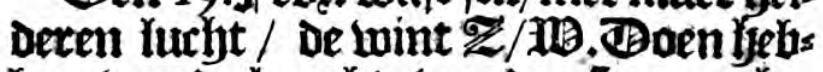
ben wa de booctjte ban der sonne ghe= nomen / Dat ug in menighen tjódt niet hadden /tomen doen / vermitgo bat den focifont niet claer bag / foa mede om daté foo hooch niet en mingh / nach foo beel fobaoume gaf als men op Ben? Kabium beborfoen / foo hebthen lup een inftrument toeseftelt / wefende een balf ront/ op d' eare belft 9o. maden gotenc= kent / baet aen haugenbe een dzaetjen met een lootien / getifit be water-parfen

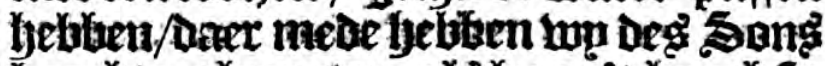

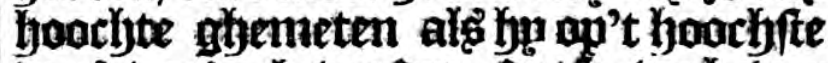
mak / woe bebonien datie berlyeben

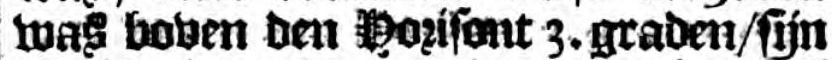
beclimatie wa 1 I. meauen I6. minuten/ be felbiglue greboeribt tot be genomen houdlyte / fo ít 14. graden 16. mimuten/ díe metrarliten ban be 90 . graben blíft de

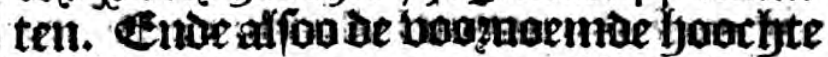
bam ozic graben gementen toen ae ans Decfte fant bes Som/ foo maet men be reffeerende 16 . ninuten soeglyen tot beg

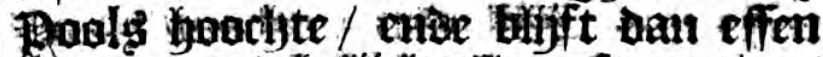
76. maden/ghelijek alfe mife meetinge

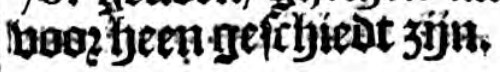




\section{Om'e Noorden na China.}

Ben 20, Fethuarij maft quate were met gewefoigen jacljt-facet ingten $3 / \mathbf{m}$. enoe waxen daex beut inetercun in't

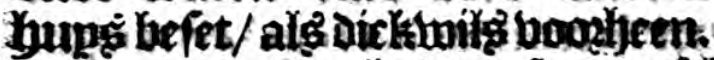

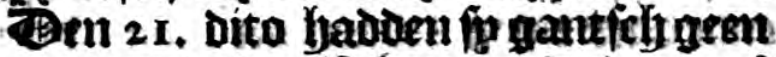
bout meex/ende alf 't quact weider was met bax be wint ende dirlte jarbt-paecu / fo moeften wo bier en baer formin bout expeng af berechen/ ende opfoerken bat onder de boten bertreden lag/bart men in oe rupmte niet op gegift en hadoe.

Den 28. baetoen pip onder haer tyie= nen noch een fede bouts met geen min= ber arbept ende moepte ald te booren/ want een ban de maet moche baex lan ger niet belpen / doog dien hem eels ban in moote teenen bet boogfte lit afgebza: ren was.

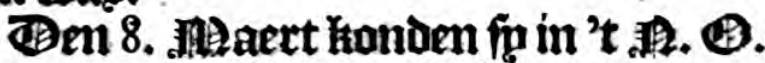
in de zee gantich geen 19 gien/waet doos fo wel bermorden dat in't 3 . (1). ban baer ren rumme \&ee wefen moft.

Den 16. Plpeil/in Der nacht quam dex cen 25 eer na th hung toe / dieg fu baer be fe dede te fifhieten / maer doog bet damp weber / enoe't tarupt bachtiont was / foo

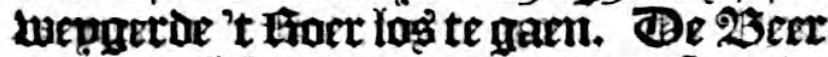
quam wel fout aen de trappen af nae de betie ban't lyupg/ ende poonge daer int te brelten maer te Schipurer hiel be beux toe/ Dieg fys te rugge fiertie. Bmtrent twee uren daer na is hys weder getiomen

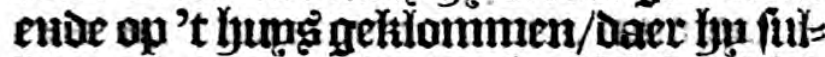
Hen gebaer maechte dat bet fofyickelijek om hooren buas/ tamende epnotight aen de Sefjoorfeen/ende daer fo grooten ge

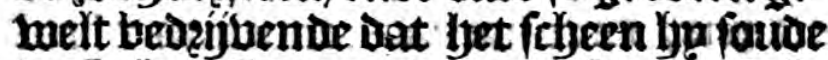
de. Selboofteen mom berre geimeten bebben / be fethente't zenl batter om baft gemaecht was enoe felde een epfielich getiex / Darf gingh ten leften wederam beenen fíng Lueeghs.

Den eerfen nilap hoochten fa baex lefte 1olopich bat fo lange nefpart lyadoe entoe was noch foo goet dat de lefte beet yaer fo wel maechte alş 's' eerfte / Doch bad enemwel defe manuel bat het niet Ianger bueren billoe. Teu 2. woept een

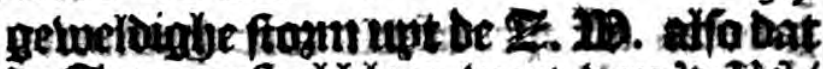

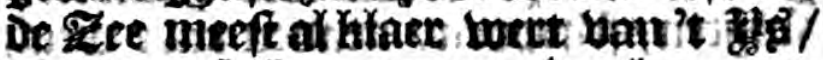
Daer dong fo begonmen te lanches nace

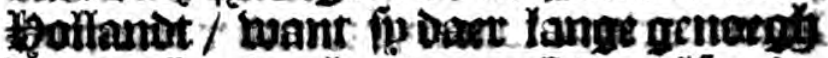
buts of henden badoen: Enve alfon to

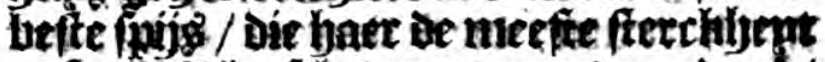

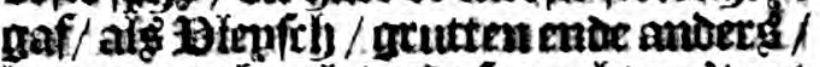

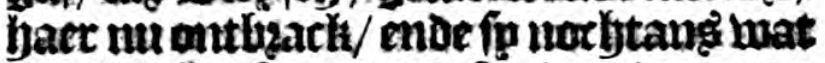
ftercklebejoefoen te weren/oun ien aens

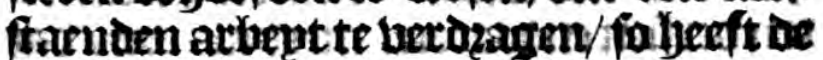
Deljipper de refte ban 't Bapech ontuet

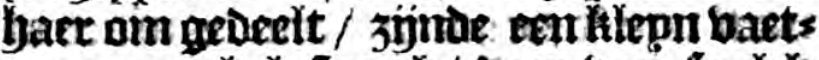
men met pekel-speck/ Daer ban hreich Fregen twee onten dargh / Deie wethen taig duerenae/maer boe bag't tmede op. Den 4. giugen fọ met haer vijwen nae't fithip/ende bonden' 't baft eontom in't ijs teggren meer ałta te baten/ want omtrent halif Maert lad bet maer 75. treden ban 't open water/ nnde mi wel 500.' 't weick

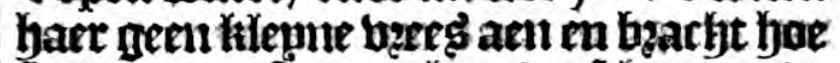

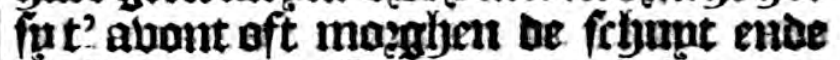
bork daer dooz of te baer nuer in' $t$ water foltoen bzengen.

Den 29. ginuen fin moer fhaer xo. nae De filnut am Die outrent bet lyugg te bertimmeren / daely bonden die gantfech diep betwelt in de fmeen / daer foffe met grooten arbent enare maepte noch unt-

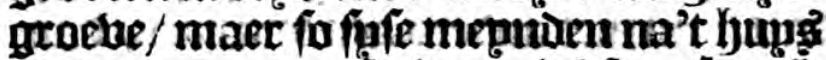

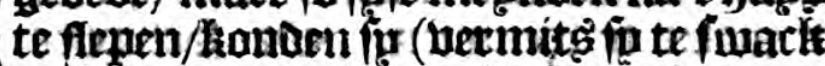
moe untueteert lairen)' $t$ fetbe niet taen daer toon ip gantfich betfift ende maes Deloog werben / Duchtenbe dat fin baer in fouven maeten blighen fteecken/ maer de Sethipper bermaendefe elck wat meer

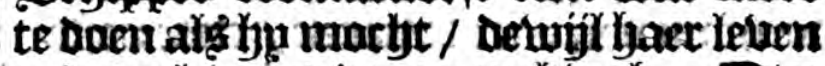
ente wel-yaert daet aen bingly. Den

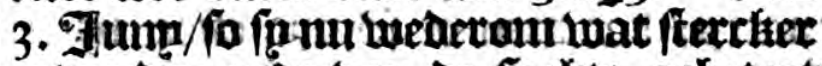
gewozoen enoe ban be fieclite sebetert waren / arbepisen fo met afle macht aen den bark / Die fig egmoelick vaerdigh iregen.

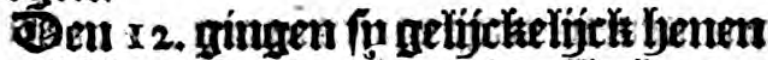
met bifien / bouncelen ende allecten wes reet/chap daer toe dienende/am den weg

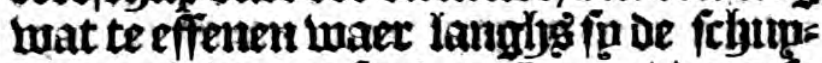
ten uae t water fouden flepen/ Daex in mooten 


\section{Derde Schip-vaert van Willem Barentf.}

mooten arbent beben met boutwen / ap't tant bejegent was / ap abontuce of Imijten / keboppen / graben ende wedh daer pemant noch namaet quame/ dat werpen. Fer woifle fir nuin't befte van ernsiertharen axhent waxen/quamber een mone-

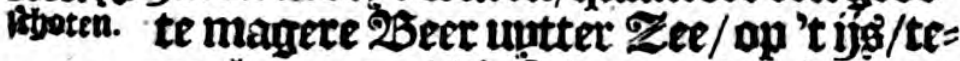
Ien baer aen / die jo bermaeden dat unt RLartarien moeft thamen (wamt fufe wel eer 20. of 30. mijlen in Zee gebonden Gadoen) ende alfo fí bam geen ingurftet= ten berfien en waren / meer alş een / Dat be 2 arbier badoe / fon lieg Veer nuetter

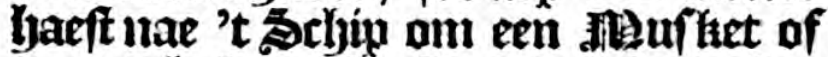
twee te halen: Be $23 e e r$ dat fiende liep hem wacker nae / ende foude bem mil= fethien achter-lyaelt bebben / maer de inats ichoten fluche nae hem tae / foo Dat ha Veer berliet / ende ficly nae de maets keerde / maer berot ban de 2Barbier gefrefooten dat hy wegh liep/ Doch Kanoe booz oat betwetlicly ende rampeligly jigs niet werth tomen / enve wert boart bont gefchoten / ald pock be tanden unt be mupl gefmeten tertwijl hy noclyleefide.

Ten r3. Itum was't noet mop we Der/ moe de sethipper is met de tim= mer lunoen nae 't sechip geraen / al= maex fo de filyunt ende bock baost ge= reedt gemaeck ende toe-geruft belben / batter nuniet andersa aen ontbark/ Dan te water te beenghen. Be Sechipuer met de gheene die by hem waren / fiende bat het open water was / enere en gaede katite unt den meften / beeft Willem/ Barentfz, die laugbe fieck geluee f twas / thenten netreben / Dat hem raetfaem Docht mu ban Daer te baren / moe befloten Doen ondertingtie met de semeene martg / Dat men de fohunt ende bock falloen te water hizengen/ende in den na= me Godtg ie renfe aenbaerden ban Nova Sembla naer lyupg tore. Willem $\mathrm{B} 2-$

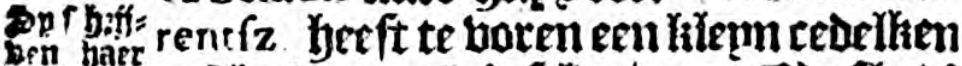

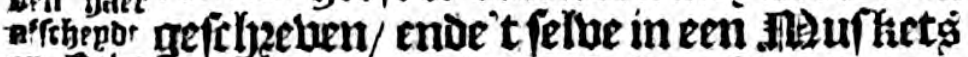
on hot a mate gedaen / in de sethogefteen op-ge= lymern; met cen kwat berhael boe fop tut Sollanot daer gefamen waren aun te 3 qulen uart China, ende wat bafr aldaex Die weten mocht wat haer bueder-baeen was / boe fin ter noot het bung gemaekt/ enoe baer thien maanden langly bupg getjouben hadden. Ende alfoo fo baer numet thee open fechupten maeften in Zee hegeben/ embe een gebartickte regre booz handen hadoen/fafitbeef de sebip= per mede twee bzieben / die fo meeft alle onderteechenden / boe fp daer te lande po lange met groot verdeziet ende ongemał berbleben badoen / op bope bat het fehip log wozaen / entue fip daer mede noch folte den twederam margen 3enten/Doch alfa't felve niet geluctien wilde / maer dat bet srtyip eben uaft bleef fitten / de t tít ber= hiep / ende haer bictualie hozte / oat fis Daerom ban noots-wegen tot harer he= bousinge't 3 ch hip maeften verlaten/en= de met de frbunten op Ébod genade nae buug zeplen, Dantefe baisuen badoer elclese cethut $\mathrm{eEn}$ in / op abontuer of fit Uan mafkanderen veroinaelden/oft boog ftazim ende omweder d' een aft D' alber bergaen mochten / Dat men dan altijot morh bn de abergebleven fethunte bebin= Den faude / hoesamig baer af ichent ware relweeft. Alae dat in nu lier in alles berdzasten waren/ fo bebben fo de fethunt mbe bock in't water gefleept/met nochn elf fleden met maet/po bictualie ende wijit als hoopmantehap/Die fo met aller neev's ftighept betiben gefoctht te bergen/te lwe = ten 6. parthens met fin wolfe Taeken / twee partiteng fluweel/thee Kaffertjeng met nett / ture barnag-tonnen met lyet

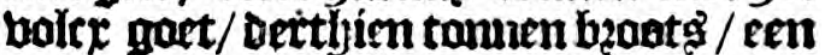

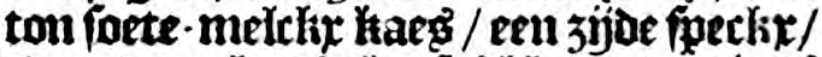

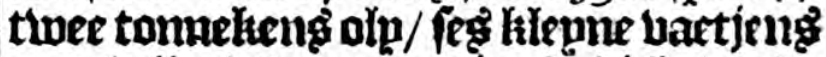
met wojn / twee baetjeng edick / ende boozta ier bondta-lunden Hilecken ende anders / fo dat men't goedt ober yoop fintor/ fouden gefent bebben / ten badoe niet in oe febuten gemacht. (Bit gedaen zijnde beliben fin eerft Willem Barentiz ,t mne daer nae Claes Andriefz die bende fieck waren / op cen ficde geflecpt nae' water/ 
Om't Noorden na China.
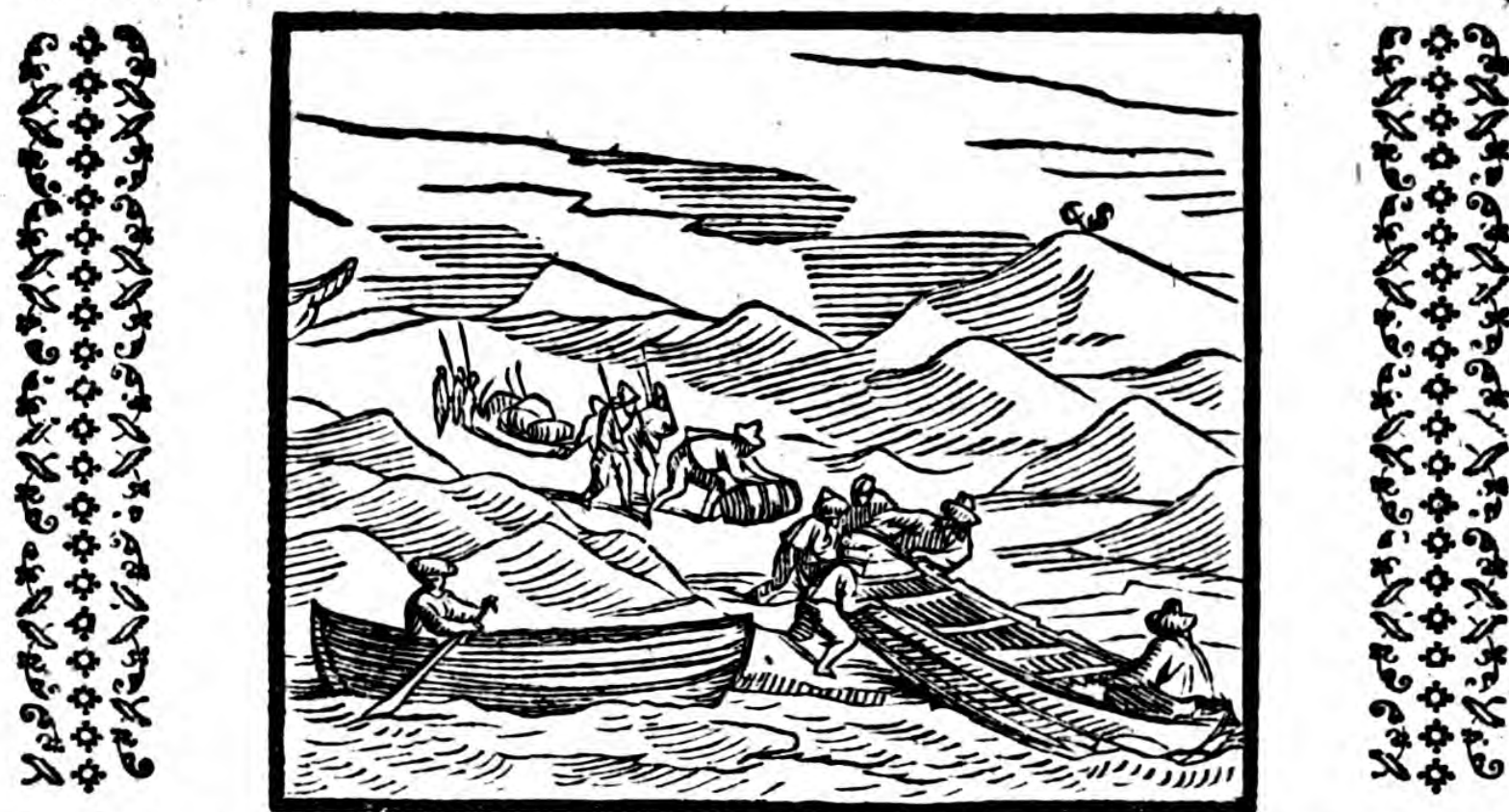

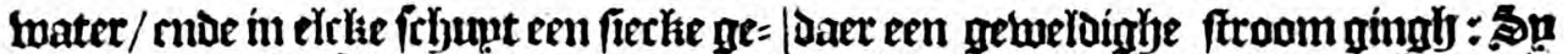
daen: Boen heeft de Srbipuer bepbe de Koockten de Bogels ende bzarliten die felyuten aen den anderen doen leggen / oen fiecken. Baer nae singen fu loeder

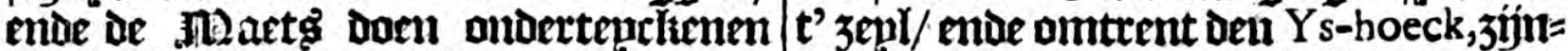

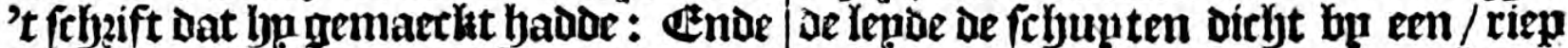

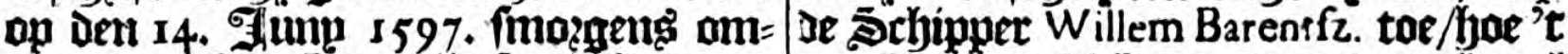

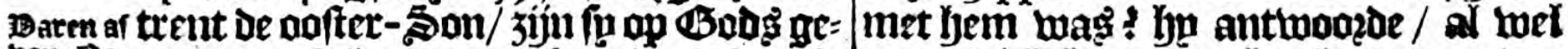
ban Ja: nade ban' 't lanot ban Nova Sembla oll 't

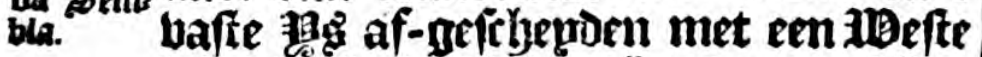
wint/3eulenide dim dagly tot aen deg $E_{y}$ lan:s-hoeck, maer lyaer eerfe intrede wage niet al te goet / want fo quamen

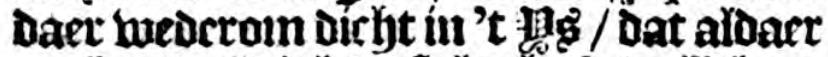
norty ueluel digh baft lagh/'t welk baer geen Glentue beega en uerooffaechte. Spp Ging:n met faer visen arn lant / om De gelegentlycut te berfpieden / en wierpen bier 1Bogel met feenen ban de hlipuen. Den 15 .'t 19 gi lwat af-relwertien zijn= De/ zeploen fu bon-bu't Vliffinger-hoof tot den boeck van Begeerte. Den 16.quamen aen be Eylanden van Orangien. - Baer gingen pp mede aen lant/maekten bier ban't houbt Dat fp bonden / ende fmólteneen ketel fneeu-water / Dat pp in tomekteng ueden om te dzincken. Dgecie ban baer ginuren ober 't 1 's nae' $t$ ander Eplant / Daer fu dzie pogejis vingben: Fin't weistom komen viel de seljupper

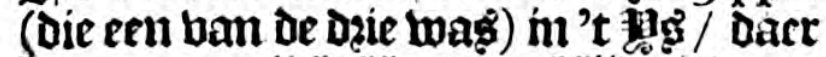
bu groot perijcket leet ban blijuen/want maet / icli loope noch te loopen eer luy te $W$ ardthuys fiomen / fegtrente boots tat Veer, Gerrir zijn lup amtrent de Yshoeck, foo beurt mp wat op / ick moet dien boeck nocljeengs fien / bier werden

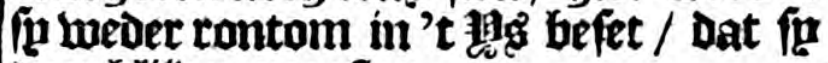
daet blijben moeften.

Den 17.'s maggengs quam't 罡g we=

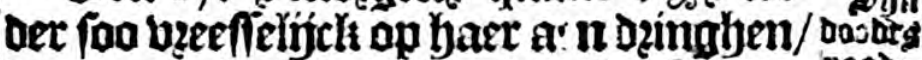
dat eener be lyapeen te berube ftonden neade. die't fagh; F̂n en homom de frbunten niet redoen / ende mennden dat bet haer laet/fe been-baerdt bedunde/ want

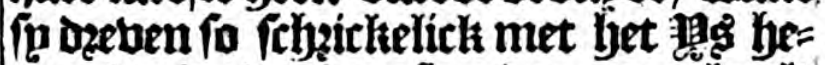
nen / moe mozden foo dapuer meturelt

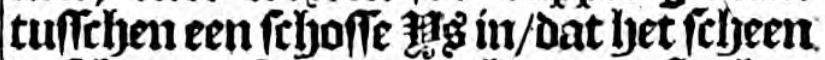
oe fibunten foudon aen bonidert fucken barften: Toaet Doo? fu de doodt elck oo: Ten-błick boos baet oomen fagen. Epn=

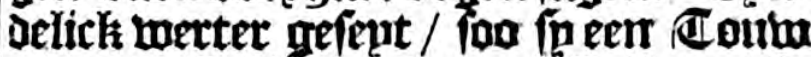

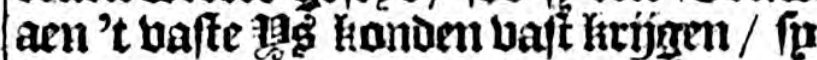
fouden de fetjunten daer by mogben ops treelten / en alfoo upt bet nuaetife um't Gaján: Befen raeot wağ wel goedt/ 


\section{$3^{8}$ Derde Schip-vaert van Willem Barentiz.}

3aer of maet niemant en bog/t be bat bel aen bertoft aet / hoeljtand eqfehte de noot dat men roozoen. 't boen moft / eurese 't meefte moft liet minfe on-Legen. Veer in befen untter = ften noot zínde / ente denclientse dat het met eell gedentitit falf goet wanben is / alg de licht fte ban allent zimo / beeft beftaen eentrog am't bafte 19 go te betumen

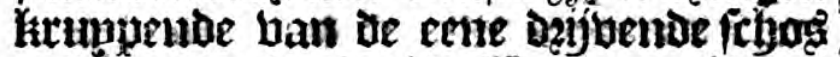
op'd' ander / enoe is alfoo doo (Boodes belpende hand aen' 't bafte Ge gelio: men / Daet lyu be trog baft marclite aen een haoren heubel. Dorn troxlten de ander die in be feljunten toaten de falve Daex bu ap/Daen tionide een man meer be= Datjen/ dan nut te booten beden net haer allen. Fen't bafte 1 gas tomende lyeb= benfe met puellex lyaeft ie fiectien daer op gebartyt/ober eentige lalieng/ntide anter aeretichap saer fu op noch ten ruften / toften baogts't anoer goet unt oe fehtm: ten / ente laeloen die mede on 't 195 / wate Doos for dat mael ust de katien Deg Doatşijn verloft.

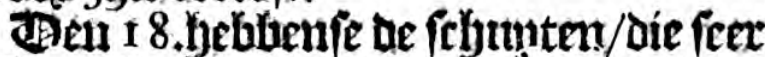
rekrentht enoe getabaertit waren/ we= Derom tereparest ende berfien / alle de

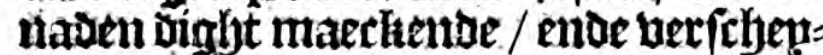
Den perfenuingen legtrentur/Daer toe haer Boo de laeete miboel ban bout gaf / am 't picls te melten / mbe alles te berep= Den batter tae diende. Baer nae gingen in te tandewaet in am eneren te pekten/ Daer de fiectien feer na betlangbon/ Dan fanden geen Gekolnen / maer binghen viet bogtelen. Joen 19 . bleben fin noch

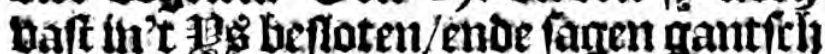

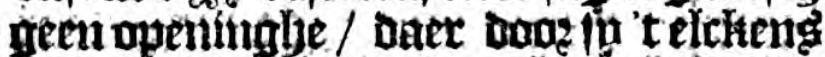

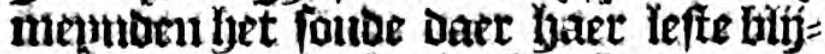
bent waroell / boch trooften lyaer weoer met t5o os tenade/ bie haet fo ditkmaels berloft hadose. Den 20. Díto/omtent 定. 0. Son quam de (sosglib-hoots man in ot lace /entoe fepoe / dat bet Claes $A D-$ driełz gefthapen wag niet lange te ful= ten maectien : Daet op W'illem Barentiz frube / mp ouncht bet fal met mp mede aiet langly breten; Te maetg yadien uevnigh bermoesen bat Willem foo

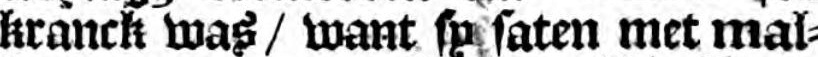
kaubeter en praten/Willem alş in Veers Caertjen noch diber ficl jeyzapooften aber ende laeder: in 't eumbe lende by bet

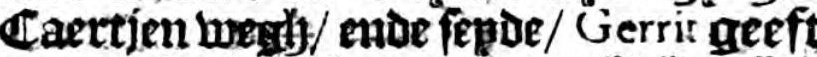
mu cembs te binclten/ maer als lyo ghe dzonften lyabse quam hein futcken qua= tijrhtyept ober / bat th fin ooglyen ber = oraevie / ende fterft foo onvoosfien bae= fting/ dat forgeen tífor hadoun de Brhip: per unt d' anuex frlyunt te roepen / ende tracx uaer hem fretfools Claes Andriefz Defe borot uam Willem Barentiz bzarfjt oe maetg geen hilenne Draef lyenot aen/ want lyp de pémcipale belepder en enige

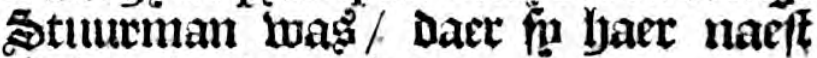
Bad op bertrantwoen.

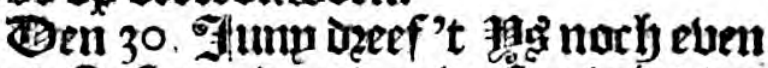
hart Boft aen boas den laeiten luint/m daer quamen twee SEeren op een felyog

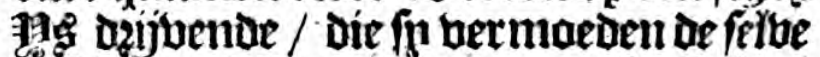
te fin ban's daegs te boorch: : an fetsen lyaer alg of fo op oe maetgan gelwilt

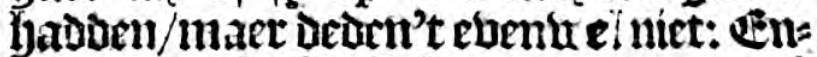
be ontrent $: \mathcal{Z}$. $\mathcal{Z}$. D. Son quam ber noct een 2 beet ober't bafte 想 aen/maer gelunt lyoarende/ ging bu wes deram beenen.

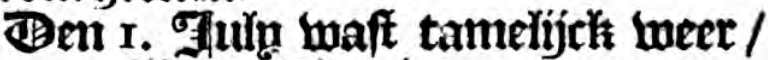

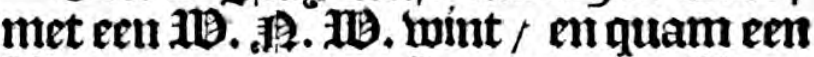
Oeer tot ong ouer f wemmen : Mlaet wo maer fiten bem doo beel roepen ber= vaet dat ly wech liep / ontrent $\mathscr{Z}$. $\mathbb{B}$.

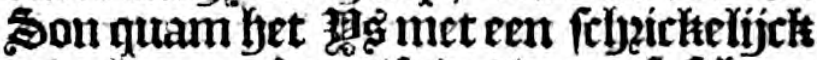

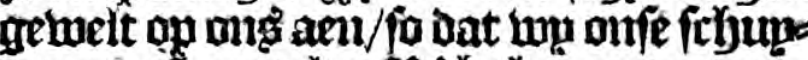
ten moeften op bet thad falen met moote maepte en perijkel ban ongi líf en leben. den leede heel aenftoot ban de 25 eeren/ die am on/e toictalie líepen / foo dat wa wepnich ruft hoften lyeblon: arae bat wp eenige dagen aldaer gefeten hadoen/ zín biff namen gegaen nae't naefte eiflant om te bernemen offer eenighe openinglye boos yanden was / want bat lange berbifiven dat meri aloaer baft faten/begon mong feet te betdzieten/geen 


\section{Om't Noorden na China}

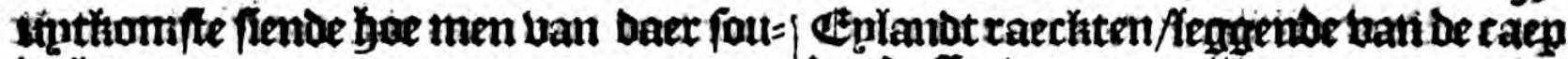
se komen.

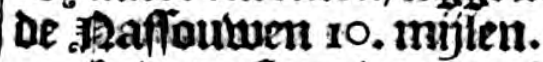

tomenoe ach be fehunten hetbenfe ons bat allegibertett/caue wo wnter noot

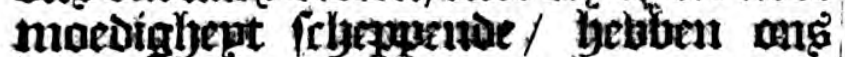
bermaent be felyahten etroe't gaet te wa= terte beengyes / ende quamen alfo met mrooter $/$ twaticheat baer ouer in't open water ontrent se 2.10 . Son. Joen

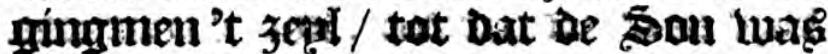

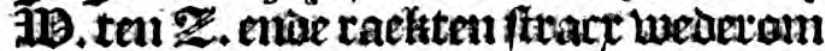

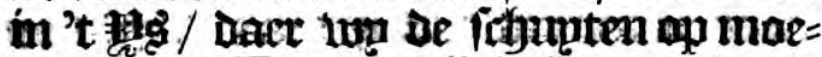

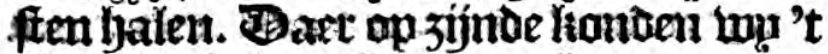

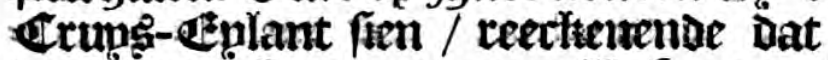
wp Daen noch onttenteen mijl af waten/ de bint wag (B). enve (1). AR. (B).

Ben 19. 'Tuly / aldug op 't ug fitten= be/zijnder ontrent ar oufter bon met bug feven mamen gegaen aen't crtmg-

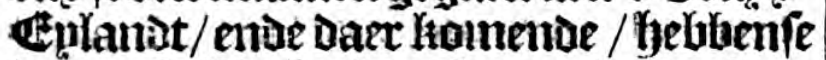
Daer in't 1 D. heel beel apen mater's ge= fien / Daer beurfe mantefts fere berbitiot waren / aljo Datfe baer haefteden watie mocligten/ wederom aen be felututen te homen/ bach raepten noclj eer/t 300 epe = ren biefe met haer namen. sten de frilu: ten fomenue/ bertelibenfe ongatye bat fo foo baet open waters hebonden fabden alfe ober fen marbten / bopende bat oit

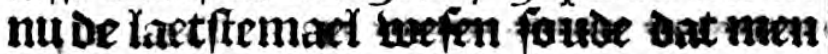
be febupten aber 't ps flepen moften/en= de dat ong beartaen 't felvige niet meex gemoeten foude / ende freatienalfo mal= kanderen een moet aen. MOp hooktten de eperen metter lzaeft/ende betien bie on= Der malfanoerea / ende tonden flure to wetch ontrent $\bar{Z}$. T. Dingen baer dich te malten/ om de falgant ende back te luater te benughen/mo. moeften Die bel 270 . terien ober 't pos trecken. Dat men met grooter couxagie Dede / am dat bog hoguten dattet de Ia:

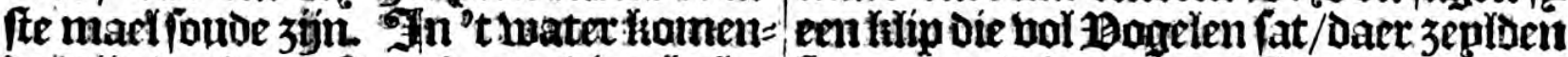
Enoer terf tont Daer na berliet ong bet pgs / Dat men Daer gants unt quamen/

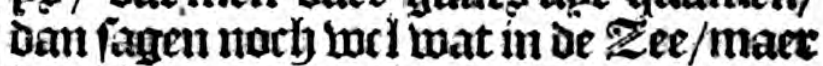
bat finderoe onge nict / ende gingen ont-

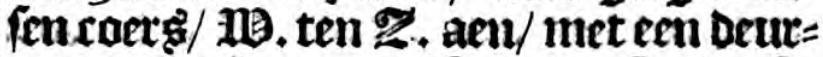

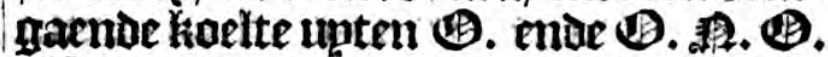
alfoo dat wy niften Dat men in elck etmaet ontrent 18. mijlen zcultbe / Dare

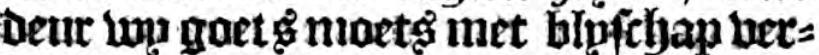
valt waren / Goot Danctitende / Dat bp ong unt foo beel f maxicheden (baet in feljeen dat men bergaen fontwe bebten) betloft enve getedoet yadoe / bettrauttwenbe ap fing goetheyt/ Dat fjn ong inoch vomeztaen gendaich helpen foute.

Den 20. Díto / hebbendenach beertíje: hen voaztgangly / zijn fp ontrent Son getiomen boos bp Den Swarten-hoek ตnde ap den abont ontrenft 1 B. Son fas

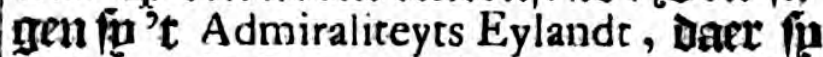

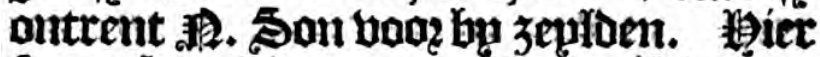
fagen fie wet bo be 200. Xibatruffetizen op een feljog 琴 / zentaen dicht la faer

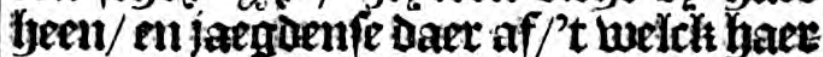
bn naef niet ten beften bertaen bacbe / want alfa't getuetoige frethe fers sijn / foe f wommenfe dapper tot yaet aen $/$ atsoffe baer Ieet badoen wile Ien weeeren/ eqtar baerden rontom de

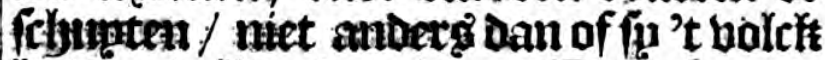
badben wiffen vernielen: TBan fin ont= quarment nach boaz de goede haelte / maer 't en was bocth niet wel gedaen fa= pende toolven wactier te makten. Ben 21. paffeer den fí Capo de Plancio, ende Langenes. Den 22. Kamende antrent Bageto Capo de Cant, is Veers uolctit aen lanot bangb. meraen / om eenige Dagels ende eneren tre foecken/ dacly en bonben der geen / mate daer nae ontrent $\mathcal{Z}$. Son fauten fü

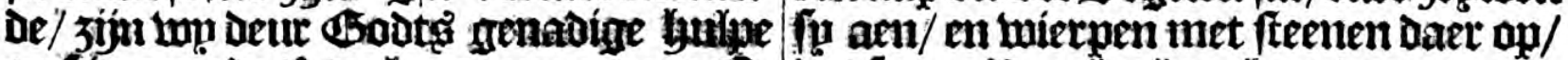

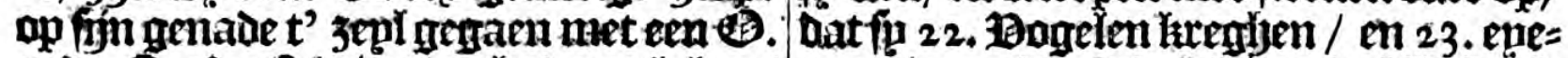

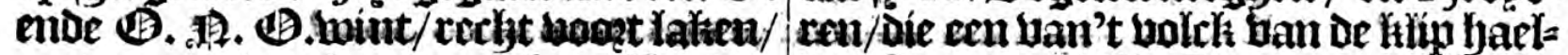

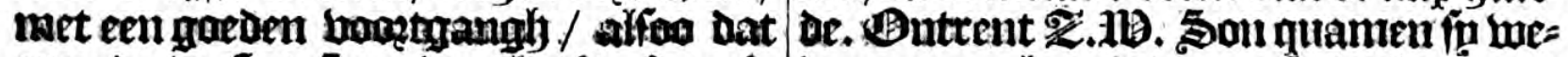

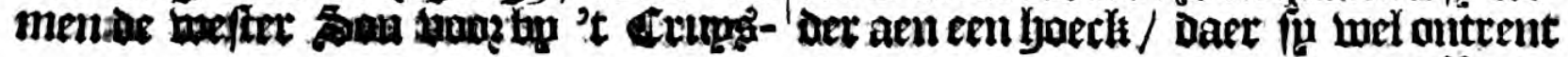




\section{Derde Schip-vaert van Willem Barentfz.}

125. Dogelen fregen / die men met De / kanderen aen't landt/ Daer for een goed hantot op lyaer neften greep/ ware unt haluen bonben/befelyermt ban meert af febeen dat fo bopeniemant beeg en bad= winton. Hier gingen /p mede op 't lan oen ban boos Boffent ende andere bilde ende raepten ljout / daer bp fp de gevan beeften / Die tegenga die booge fteple hlip= men Bogelen hoochten. Den 23. Dit pen niet op en houmen / wait ljaddenfe waft bonicket ende miftíg beder met ce

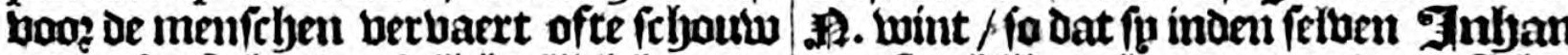

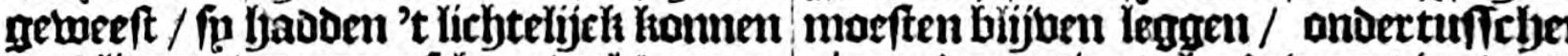
ontuliegen / maer mufbeen wel/waten= gingender enige te landelwaet in / eno

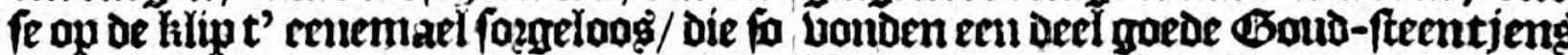

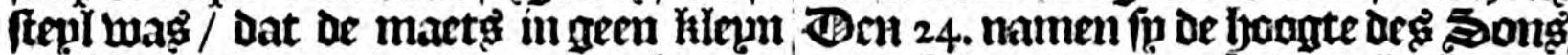
perijekel en waren ban armen en beenen enve bebontoen bat fn daer lagben of te beeken / In fonder in't af filimmen. 73. graven 10. Inin. Sy moeften noch a Defe Bogets yadoen elck maer een ex aen ftrant blijben/ende gingen al weuer in't ueft / ende dat op de bloote lilippen olu fteentjeng rapen / ban de befte die f

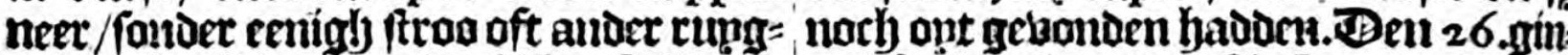

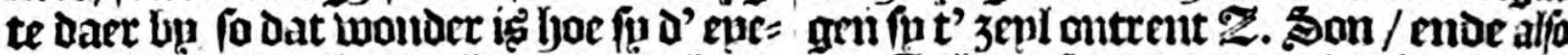
ren in fodanigen foube haumen unt

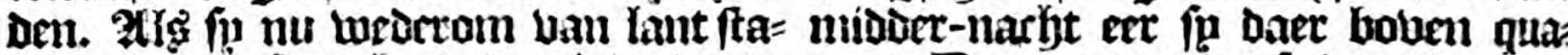

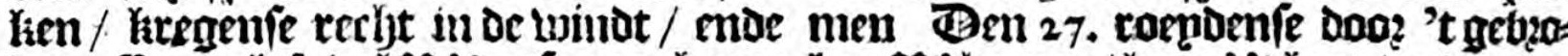

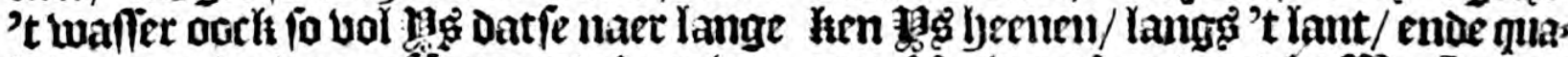
laberen ende bergeefie moente daer we= men's abont ontrent De A1. Saiter

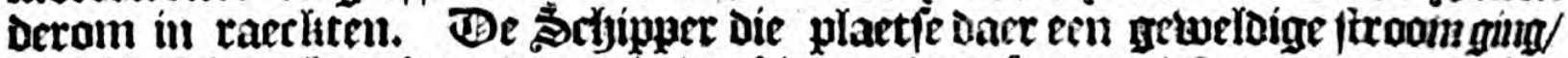

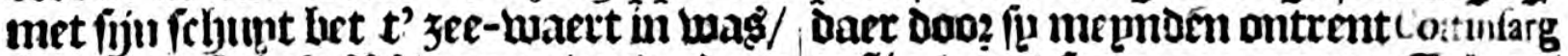

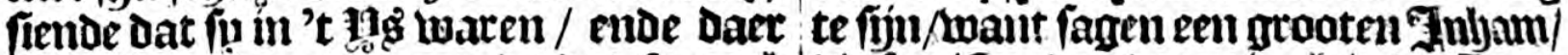

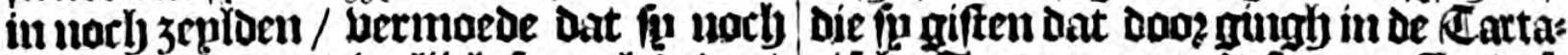

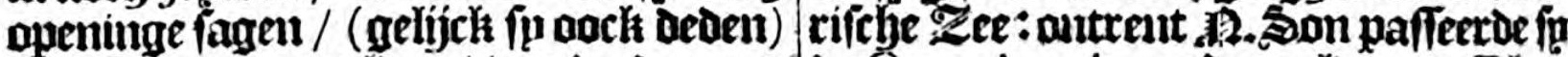
Daer fn nae toe zenlden / wende daerom de Cruys-hoeck, ende zeplien tuffenen mede na baer tae / ende quamen Iṇ mal=/'t vafte lamt ende een Eplanot Doos.
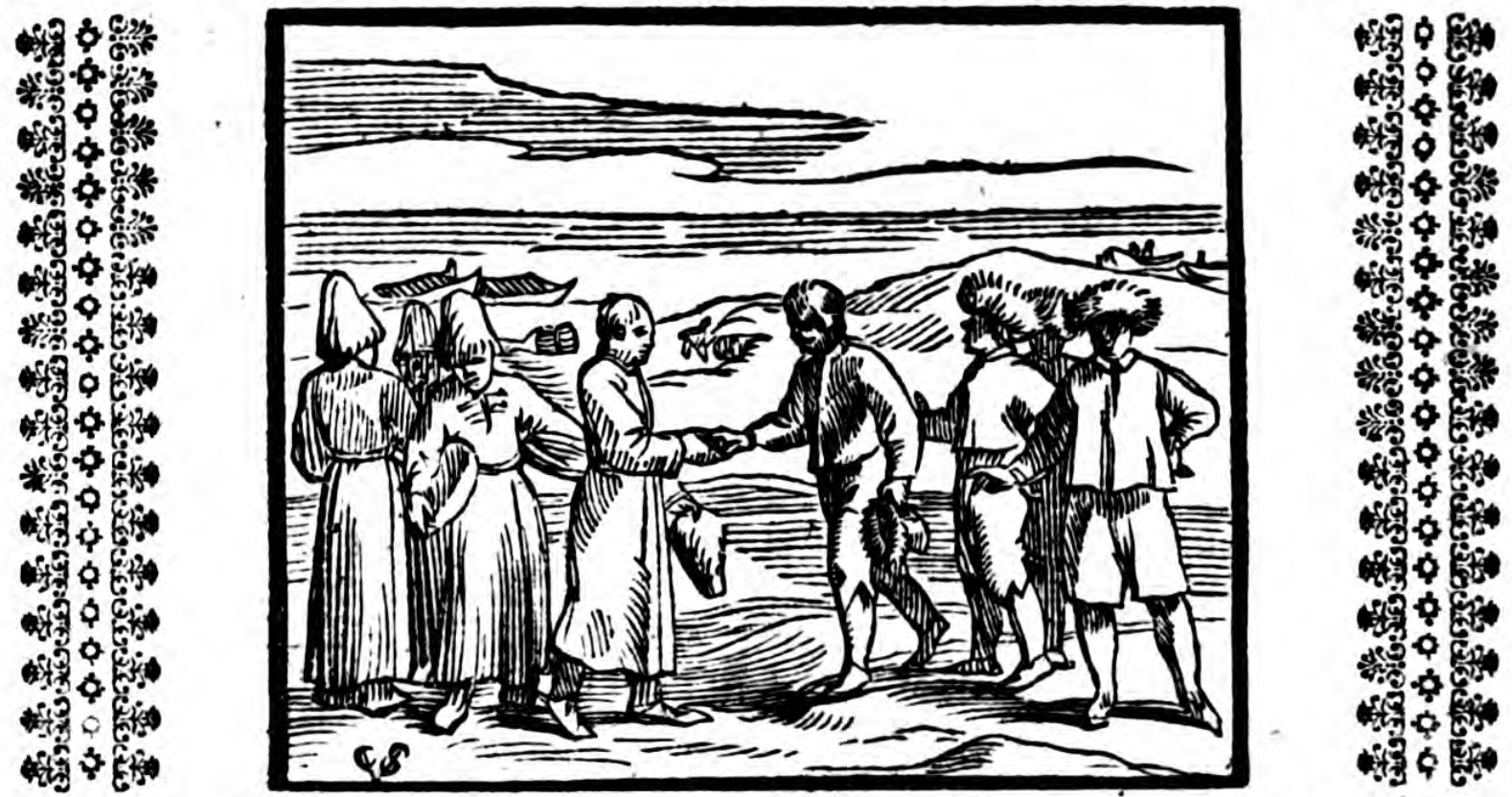

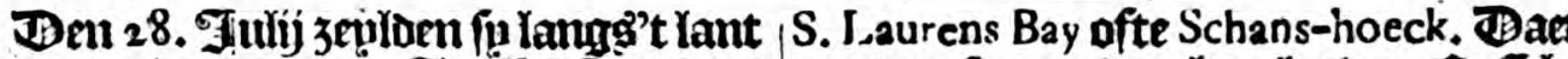

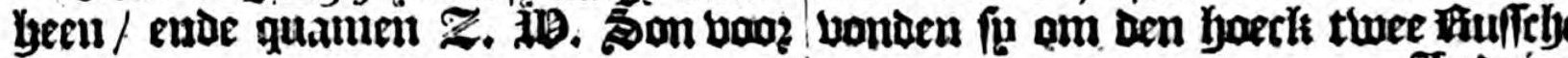
Tougien 
Om't Noorden na China.

eprin: Todnien lewgen / waer Dooz pp ten Deele oen rwee berblíjot waren / Dat fperng ter plact fe cotgren. quamen daer fo menfethen bonden/maer ten Deele waren fo ouck beoucht/om dat Díe sllanfichen wel dertigh fterch wae= $\mathrm{ren} /$ niet wetende wat bet bous bolch mocht wefen / abilte of andere ondunt= fibe. De tholfanors quamen met groo= ten arbept aen lamot / 't toelel' de thuf= feben fiende lieten baeren arbeyt ftaen / ende nuamen nae de ollaniers toe/ boch fonder gelweer. Jis alkanderen ge= naeliende/ de den fp aen weder zíje/ elch th op fín maniere/ malkanderen groote eerbiedinge. Sommine onder baer wer= Den de onfe hennende/ende fauenfe deer= líck aen / waer ober be onfé haer mede quamen te kennen / ende fagben dat bet de felbe waeren / die de reng te booren ( Joen fu doo? de Weygats voeren)in baer Schíp getweeft hadoen/ ende daerom/ po pipel mercken fonien / ober haer ont= fet ende befommert waten/fiende dat fo nu fo mager ende ontftelt/ met podanifre open felyuptjenge daer quamen / merben/ die fo doen fo mel geftelt met foo lyeerlije= ken Brthip / ban alles bel berfien / ge= den hadoen.

vouben praerta mut be
Bnder haer waerenter twee die den Srfyipuer ende Veer bifendelijelt op de

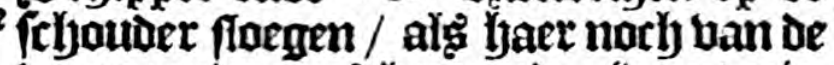
boozgaente rengs łannende (wait nie= mant dan fu twee en haider doen ban de onfe in De Weygats gelweeft) ende bzaeg Den haer nae haer Crabble, of Sorhip. Sn geen Tolch helobende / beounden bart Dat fị 't Scljip in't pos berloren badoen / waex op de suffeben enuden/Crabble

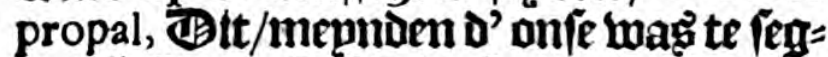
gen/hebon't Sechip berlooren? ende ant= woozden Crabble propal, jae hup bebben 't berlooren. Doen bedunden fo / Datíe met ong wijn in 't Selhip gedzoncken badben/ Daer buer dat een ban de maet in De feljunt liep ende tapte wat watersi/ bat hp haer liet pzoeben / maer fo felyul= Den baer boofoen / feguende no dobre, dat is niet goet. Daer nae is de Schip=/ringe/maer bondenoer niemant. gbemaechit. per baer naerder getreden/ moe heeft hem in de mont laeten fien / om te ken= nen te geben dat ly met de fíne ban't Fefyetr-bunck gequelt wage / entoe of fugeen raed daer toe wiften: Sn ver= ftonden dat de onfélyonger babien / $\mathrm{en}=$ de een ban lyaer liep ter font nae be Tlod= gie / balenoe een ront roggen-23zodid ontrent acht pont flwaer fommigbe ge: roochte Dogels: De Getyipper danchte baer / ende gaffe meder eentyalf dozín befljunten / Iendente twee bau baer nocbeeng fofbenckende ban de ABijn die bu badoe / ' ander marts gingen daer ji lagben / enoe koockiten bu baer buer wat befchupt met mater / om wat warms / in't líf te latígen / wel blijoe zínde ende God danckende dat fy' 't eer= the in Dertfyien . Ma aenden eeng wederont

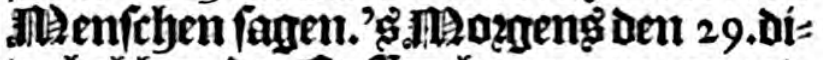
to lyebben de fufien begromen gereet: fibap te maechen on huegh te zenten/ gravende unt den ringel pommige tan= nen met Juraen / diefe daer onder begra= ben Jadoden / ende nut' fofyeep bzachten. d' Onfe niet wetende waer fin beenen hildem / fagen datfe nae de Wergats lie= pen / daet ober bebbenfe mede zenl ge= maectit / ende volgboen yaer nae / dan 't werot io miftigly ende mottigh dat in malfanoeren quijt werden: Goch jijn d'onfe ebentuel boost gezenit tuficlyen de

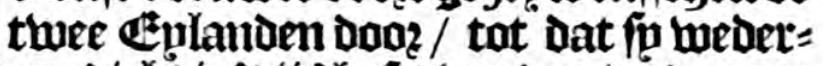
om diclyt in't ýge befet merien/ende geen openingen famen / waer doo? fug bermoe: om ontrent Weygats te maeten $301 n$ / en=

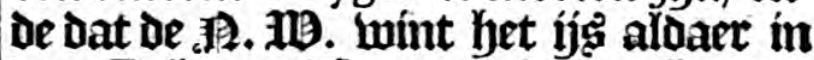
den Infyam t' famen gedeeben hadde: Sín daerom met groater maepte we: Derom te rugge gebaren tot aen be twee boomoemde Geplanden / ende bebiben aen' $t$ een ban dien lyun Schuten baft

Den 31, roepuon fọ ban dit Eplant na een ander daer twee ftumfien op fon= Den/ daer booz fu mepnoen dat albaer eenígb boilk gelegen hadoe op haex nees $\mathbb{J}^{2}$ 


\section{4i2 Derde Schip-vaert van Willem Barentfz.}

xest:. Sygingen bier t' haren grooten ghe: blaperen Yuck op 't lant/ want bondender II bladeren / die baer Lomberlích wel te paffe quamen / gemerekt fu beel fiectien Hadoen ja meeft al/ alfóe ban't felyeur= buncle geplaentht waren / Dat pu naulwe= fijck boost mocliten. Spaten defe bla= deten by band: vol op/want fo inteollant beel lyadoen booren feggen ban baeren krautht/maer bebonden die meerder dan fu geboot oft gemeent hadoen / ende't hielp haer foo merchelích ende lyae=

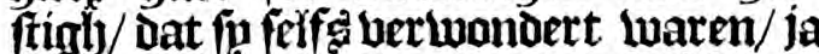
fommige aten terfont weder befrlyupt/ Dat fu katats te boaren niet ljadon hon= nentom.

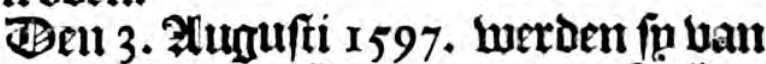
enp fes beraet lan Nova Se mbla ober te fteetien

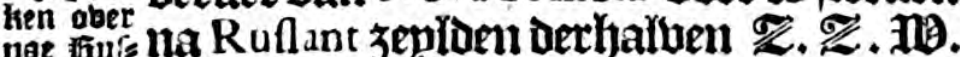

lanot. aen tot De oofter son tae/ende quanren Doen lueverom in bet jja 't weicli ljaer gant/ch bebeeft marelite/ want pin bad = dent al obertefet ende adien tilyefpet.

Ditus in't ijg fittende met filte/ raep= Den fiu met grooten berdietiuen arbept Baer Door/ende raeckiten ontrent $\mathscr{Z}$. $\mathfrak{1 1}$.

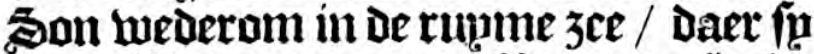
geen íg bernamen : 7 oozt-zentende meunoen fu de thufe liuft te genaeriten/ maer ontrent De $\mathbf{3}$. 1 W. Son raeckten fulneder in't ijs / Daer dooz fo gant/fy berflagen waren/duchtende dattet baer altýt aenfjangen foude / ende dat pug nim: mermeer daer ban foudé ont flaten woz= Den: $\mathbb{e n d e}$ alfa fin met de lock niet wel boot konden/ nock den froectit ban't ijg te boben zeplen/ werden pup ged wongljen faer dare in te begeben: Thaer in zijnde hadoen fi 't luat beter / ende quamen met mooten arbept ín't apen water. De Srlyipper / Die in d' ander feljupt hage /

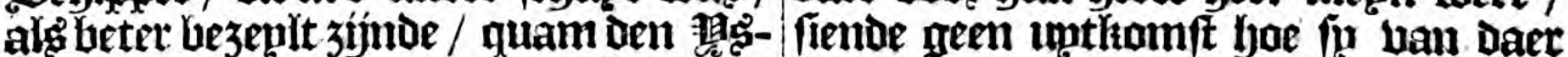
hoeck te bouen / en quamen alfo weder = fouden ralten. Te fieckte ende honger om bumalfanderen. Den 4. Dito ons berteerie feljier haer blenfth ende bloet :

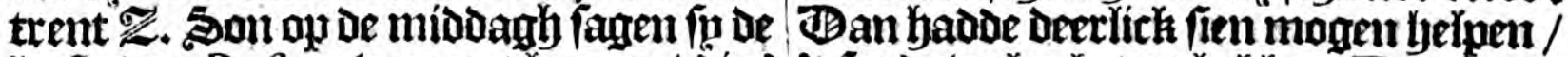

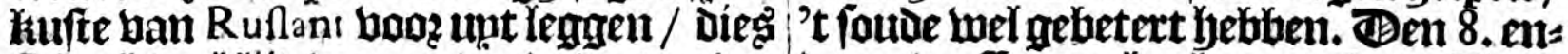

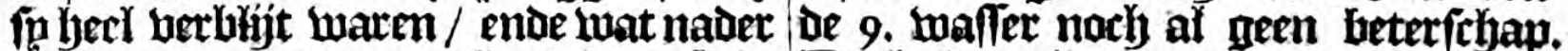

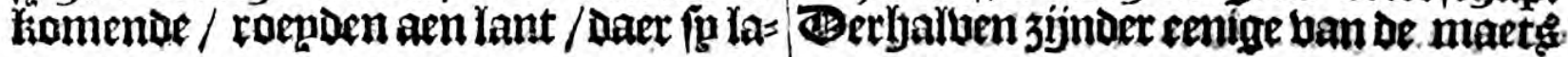




\section{Om't Noorden na China.}

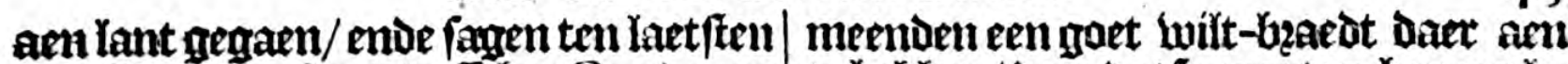
een 2 Baecken ftaen/tuffeljenCandetioes endoe't bafte Laut baut haffiant/ daer tunt fin bermaeden dat bet de tours hag daer be fiffen lenen quamen $\mathbf{I}$ ' om hamen bondenfe moer wegen een

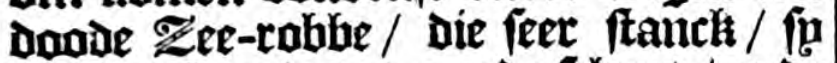
te he haben/ Dooz datfét uroaten houtger Yes

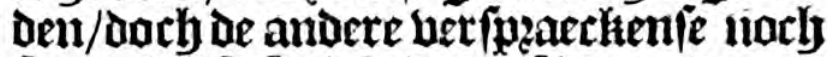
feggende/fú fouden Daer Dé doot aent eten $\mathfrak{m}$ Daerom liever noch Lwat Yijden/moge=

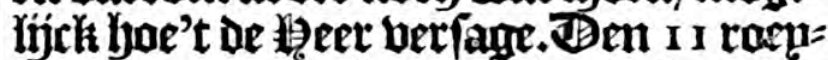

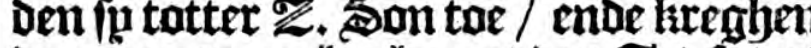

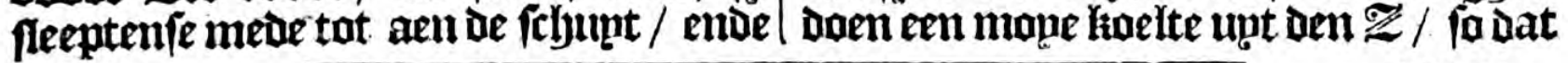
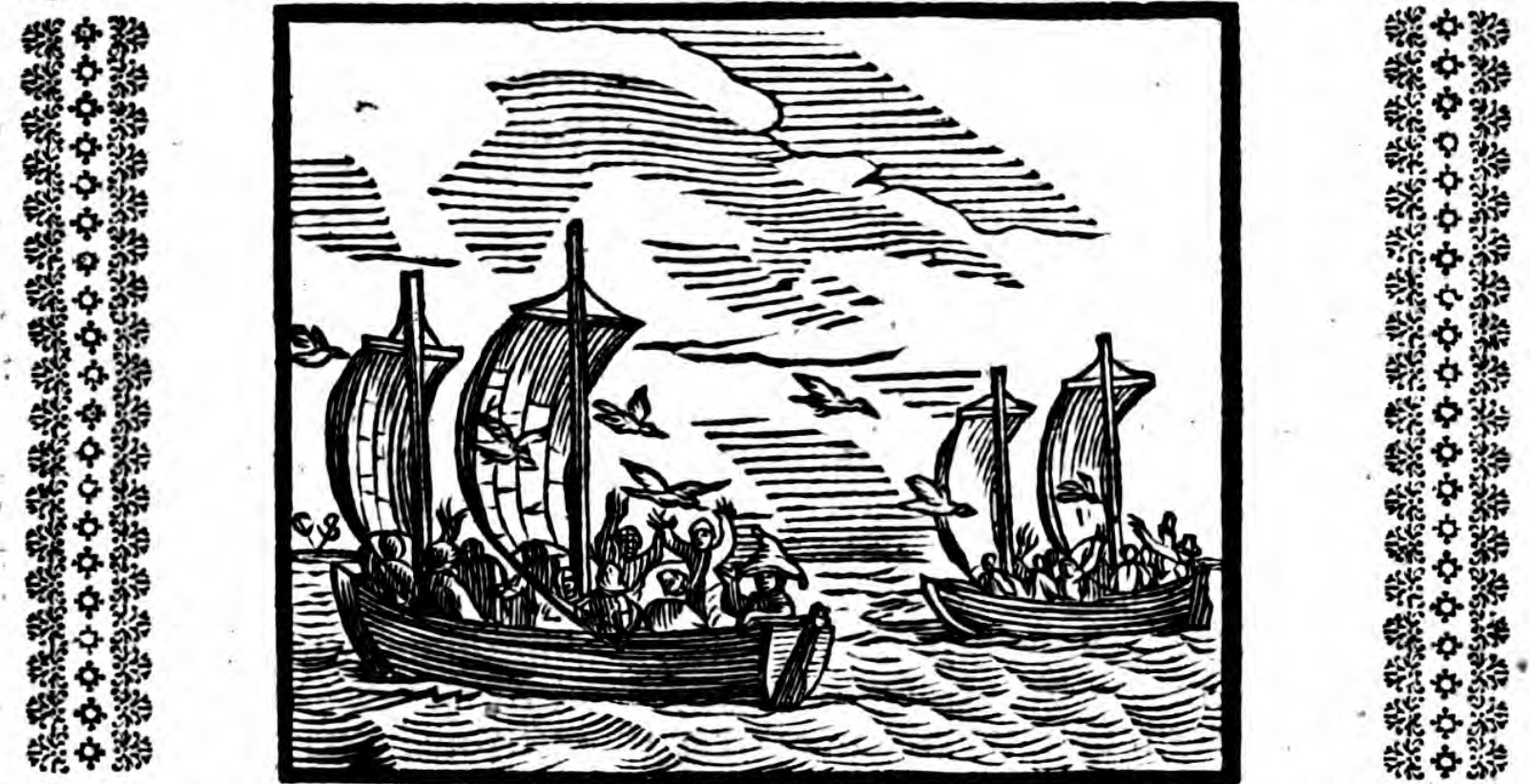

for 3 eñl maechten met een gaè̃ baostgamgly / en kreglen 4, a 5 . Dogelen foa

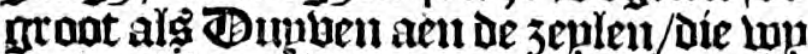
met onfé ljanden meven / Dat put wat berlyeugoen / maer fabontge begont yart

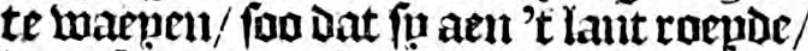
daaer fọ unt ginuge am ber

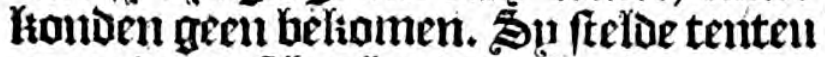
om onder te febtulen luant 'jet begon ge=

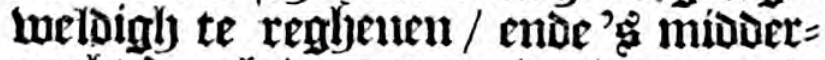
nacht to oret Dapper te tonderen ente Ghlixemen/ Doo alle 't welche de nraets wederom lijfter mifímaedgly waeren/ fiende datter nacly geen ont fer vooe ogen wag / ende fommiglye luenflyten om baet boot.

Diten 1 2. dito omtrent (19.5on fagen=

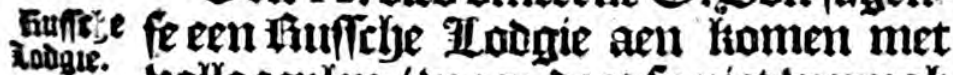
bolfe zenten / waer Dooz fu niet buepngh berbihjot waeren//yaer baeftende wat fo morljten am de fibunten in't diep te frijen/ende daer na toe te 3 eplen. 20u yaer kamende gingl de feldiper in de

近odgie/ vagagende lyae berte funocl ban Candenoes maerel / maer Kande fuldetip

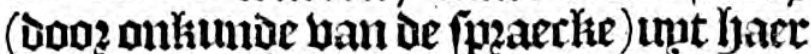
niet berftaen : Sp ftaection biff vingeten op/ 't welchs de onlfe baer lieten Dutucken fo beel te hesumben/ als datter bijf Crupfien op 't lät ftonden / bzarbiten

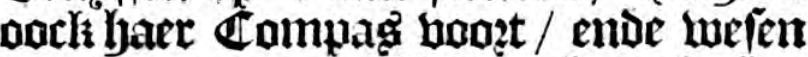
Dat het lanot A. ID, ban yaer laygl / 'twelf de onfe op yaer eompage me ue al=

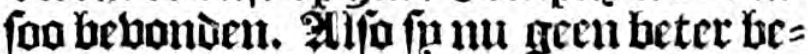
frabent bande laffen kantoen bernemen/ traot de Srhipper Lwat nader:ende luees haer een toune met bifey die fu yadoe/ bzaglyende of fin die bifton bertinapeny/

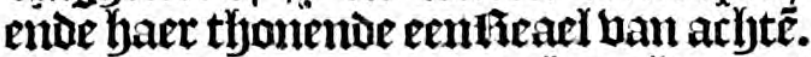
Sp Dit berftaentwe/ gaven bem lyontuert ente twee bifichan/met fommigre katex= Geng die fin ban meel aefouen hadjen/in 't kolten bau baren bifkly. Omtrent $\approx$. Soul februden de onfe ban haer / wet berblijt jijnoe datfe wat bictualie be= kamen badoe/ want f́n bawden langen If 2 tijot 


\section{Derde Schip vaert van Willem Barentfz.}

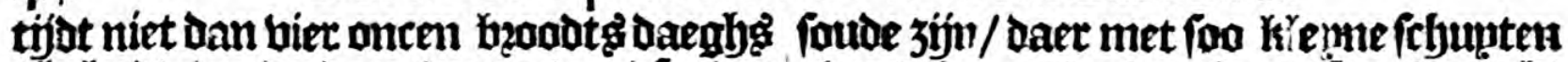
uhefhait / ende daer water toe / fonder pets ter IBerelt meer. De biffeben deel= den pu onder maffamieren om/elch eben beel / poo wel de minfte alg de meefte/ fonder mider feljept. Den 13.gingbender twee ban de maet aen lanot/om te ber= nemé of de hoeck bam Candenoes aldaer in 3 ee ftrechte/wederam fiomende/ $3 \mathfrak{e n}=$ Den/fn ltonden anderş niet bemerclien/ of't was de ljaeclis die fin glyemeent had = Den. THaer aber in alle garts moet zijn= de bederom in de frljunten gingen/entde raepde fon bn't lant heenen. Beg na=

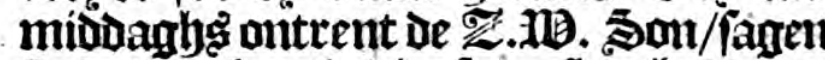
fo dat de fjoeck die fin gefien hadoen/ ontviel nae't zupien y daer booz fin nu boaz getwiag hielden dat het de haecti bam Candenoes was / van daer fu mennoen te zenlen ouer oe mont van be Mitte

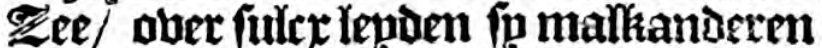

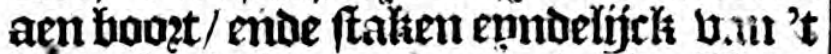
laint af / aldis meenden / ouer de HBitte Zee naer lfuplanot toe : Allougs zeplende met een gaede bnotgangl iffer omtrent midiernaclit een groot onweer unt den nowien ontfaen / waet boo? de twee

ABe twe itbupten berdoos Yen ban maikan. ereren. fehunten van malkanderen berdoolden. Den r4. bermont beel op te klaren/ po dat die in Veers fithupt waeren baer anoere maetş effen fagen / en deden alle neer= ftighent om bu lyaer te kamen / maet konden niet / obermits dat lyet miftigth merdt. Jen 15 . boozt 3eplende met een mope ketelte / farren fin outtenot be $\mathscr{Z}$. Son lyet lant/ende meenden dat pp nu

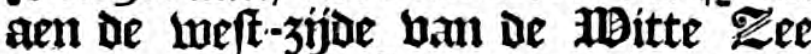
waren luga bp Candenoes, Kamende diefht onder't lamd / faghen fo daer fege Ifufteye ILodgien legren/ Daer fona toe 3eulden/ ende bagaghaen de fhuffen bae berte fu noch ban Kilduyn waeren: (Tie bedumden haer / datfe nach berde ban baer waren/als noch zínde aen de boftzijde van Candenoes, fuymeten yaer yan= Den in een/en willde daer mede hedupdē/

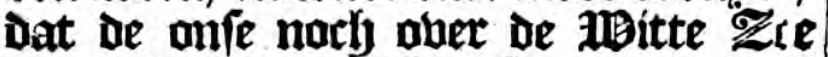
moeften/ ende dat bet feet periculeug ober te baren/gaben de onfe gebzarght zínde/cen bzoot/ Dat fu alfo dzoogb met goede fmaeck al roeuende op aten/maex fonden niet ghelooben dat pu noch eerft daer omtrent waren / want lieten baer Dumekten alober de witte Eee te wefen. -Ben 16 fetteden fu baer rours na een fiuficlye Iodrie tae die fa aen fuurboost gefien ljadoe daer fin met gronte moepte bu quamen / bragende baer na Sembla de Cool oft Kilduyn, maer fig febudoen baer hoofoen / moe gaben te kienmen dat lyet Sembla de Candenoes was: : Dit en uelon foe de anfe niet/ enve betgerde ban baer eenige fujife/ py gaben haer een deel Reljollen / daer de sotjips per haer een f tucls geltg boo? gaf / ende zeploe de onfe doen van haer af om doo? 't gat te komen daer fé voo lanen/alfó

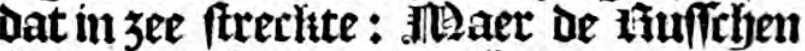
fiende dat fig op onwegly waren / ente dat de bloet meeft berloopen lwagh/ fondé ther mamen inet een groot broot in een Klleyn Flolletjen nae de onfe tae/ende bedupoan baer / fig foube luederom aell hatr fehip fromen / om maffanderen naeder te anderteclyten. De onie galuen haet een ftuck gelt met men ftuk timuen/ maer fu bleben al bu ongs / en vie ban de groote Il oogie ftaken fueclit en boter om foong/om de onfé bu lyaer te locken; fitomende bp bart / baetien de onfe baet Caett booz den danly / iwaet upt fot haer onterrechten/ dat /e norly aen be

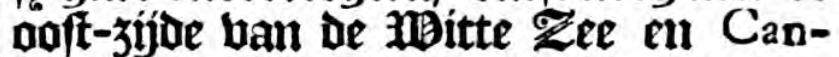
denoes waren. Bit berftaende / waren de ourfe feer beducht/ dat ṕn noch fo yerre repue te doen lyadoé ouer de Mitte Yee/ maer alletmeeft om baer andere mar: Kergi die in de bock waeren : De schiuk

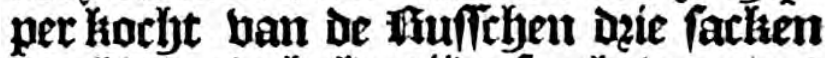
ineelg/ darde balbe zíjoe pueck / een pot met tuffebe boter/en een tonnelten met fonigly / tot pzohiefie fo van fín bolelt alt ban de andere maet of fig weder bu een quamen. Intidoelertijt was de bloet

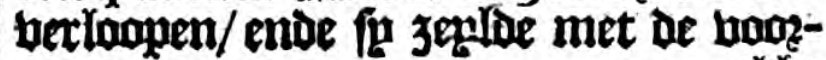




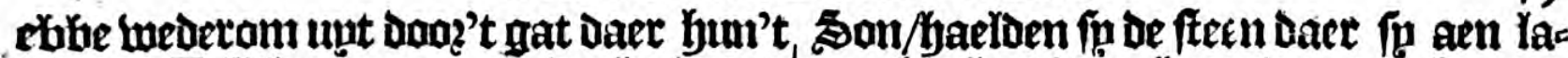

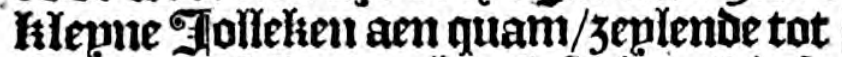

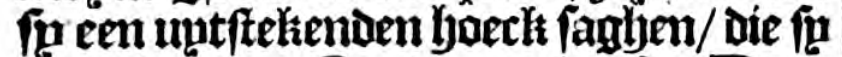
meenden dat Candenoes was: Daet Gleben fp op den abont Yegren/en Kaock = ten een yot uol water en meel / Dat haex feer Leel fmaeckte/om Dat Daer wat 'pek

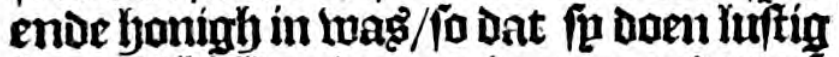
Kermisa ljielden/ maer waren ondertuf/" felyen befammert met baer andere

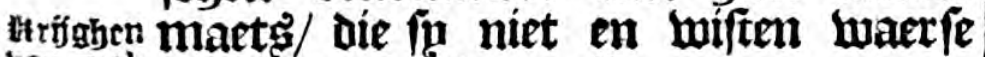
ba:l aal morliten glyebleben zijn.

Den I 7. aen't ancker Yeggende fauten fu' mozgeng in Den Dageraet een ufut

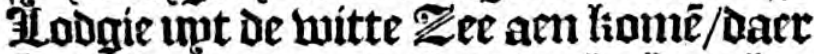

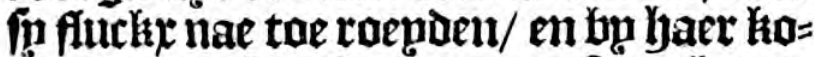
mende krege terftont ongeep ft een bzoot:

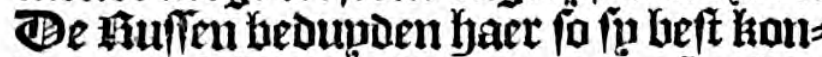
Den/ Dat fur baer andere maet gefpzolten hawden / en Datter 7 . mamen op lwaren.

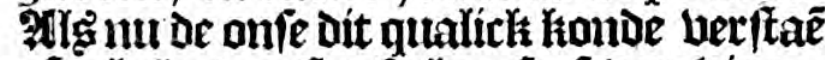
oft ghelonusen foo ftataen fó feben vinge= ren ap/en wefen ap de fétunt/baer mede betenktende Dattet mede fodanigen open

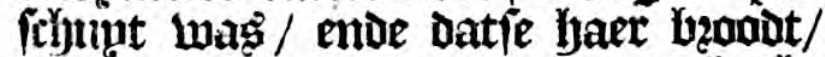
Wlengs / bifth en anders meer berkectht hadoen. Toe onfe betblifot zínde/ werde bp haer een Compasije fiende/'t welle fis

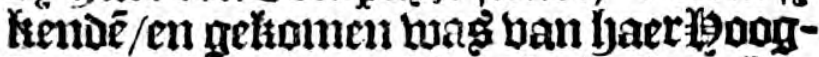
boot - -man / beapghoen derbalben boe lanye bet geleden has dat fip de maets Gefien hadoen en lwaet ontrent; $\mathbf{S} p$ be $=$ Duñen/ De daegrly te baren/enoe Deden de alle alle cre ende beiendtichap / die haer mootelijech Dauckten/ende twel ge $=$ indet fínde dat ip tijoinge badoen bam fate markerg/te meer om dat be felve fo wel gefpijit waren/ daer po alfermeeft in hefiommert waten getweeft. Eoep= Den derthalben met alle blijt na lyaer toe/ of fofe betamen ftonden/ ende den gant= fethen darg geroent hebbende lang; 't lant yenten/ bonden antrent de midoetnaclyt een afwateringhe ban berfich water// Daerom fu te lande gingen/Gaelden wa= ter ende kregen mede eenige lepelbladeren.

Den 18. 's mozgeng ontrent de O. te lande nae de buyfen/ daer bum groote fien foe lyp aen frepoe zijoen onthail/aen o'een in 't $\mathscr{Z}$. (1). enue aen i' ander $\mathscr{Z}$.

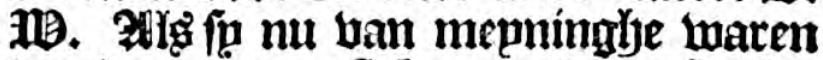
van daet ober te ftelten ulae de weft-zijoe ban de witte Tee aen de Tlapiche kuft/ bebonden fu dat lyaer eetre baetgen met water meeft untweleckt wat? / ende ber = míts ju wel 40. mílen weegts te vaeren hadoen / eer fa fu eenigh verfoly water fouden Kamen/ focliten fu norb eerft aen lant te roenen om lerfety water te balen maer om dat het rantom fa feer batren= de/ boeften't luict beftaen / en uíngen ap Gasto genade t' $z \mathfrak{e n l} /$ maet kende een af = ftercker ontrent 12. 19 . Bon/ende 3 ent= den dien gaut flyen narbt/atge mede din daglj daer aen bolgenie met foo goeve

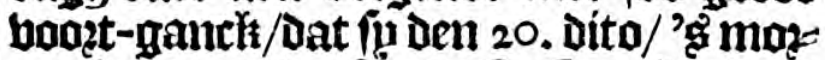
gengs ontrent de (1B. fagben aen de weft-zijos ban de Mitte jee/'t well: fu donę't rupfien ban de lant5ee gewaer Luerden/eer in't fagen/Dan= fende Joot dat by ljaer in dien tijt ban

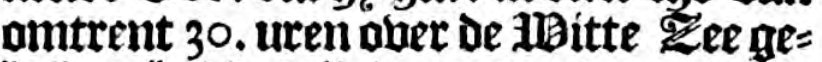
bolpen yadde/ jínde onttendt 40, mij= Ien wergly?.

Boo?'t lant wefende / ende fiende niet beel boozded ta te homnen doen met barê/ zín fo daer tuffenen eenige falippen in. aelopen op cen gaede reede / en een wen= nigh Línnen komende/ fagen een groote Ififfe Ilosgie leggen/met nocly eenighe hunfen daet menflyen in waeten/ roeps Den derljaluen na de Ilougie toe/maeck= ten faet feljunt daer aen baft/en mingen men/m plaets ban haer merp-antzer op/ ende roepden al langbe't lant henen tot de $\mathscr{Z}$. Son toe/ Doen fauen fo een unt= ferckende boeck / met febemeringe ban

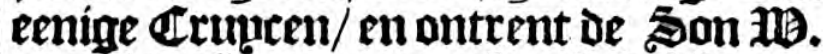
merektenfe aen de tepkeng befrbepde= lick dattet de hoech banCandenoes Lwa:/ aen de mont ban de witte Eee / Daer fu langhe nae berlanglit hadoen. Defen

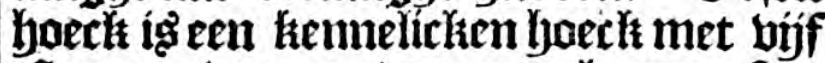
Crupen daer op / en men kan perfect $\mathbb{N}^{2} 3$ byients 


\section{$4^{6}$ Derde Schip-vacrt van Willem Barentfz.}

bzient flyap gefthieden / want werden in bareftohen gelept/Daer fo haer natte hle Deren dzoougdé / en boozt

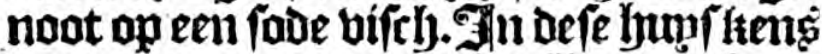
waren 13. manmen/ die alle mazglien met ture febuten boeren unt vifichen/ Daer twe ban liaer lyet belwint ober lyad= Den. Sp beljielpen haer feer foberlick en=

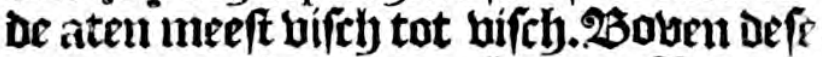
13. Inamen waren nocls twee Tlapen met deie bijuen/ende een fint / die wel dapper armelich leef dé ban't ober fetjot Dat haer de huffen gaben/ fo een ftuck bifchende eenige yoofden bie fu werth wierpen/Lellie de Jappen bau met grooter danckbarthent op namen / io dat fu ban loegen faer armoede/ be onfe boe fober fo 't fetwen marbten heblen/ norb tat medolijoen helweenboen/ ende beel Deden ont fettell.

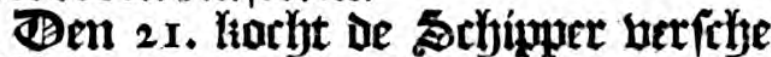
vifty/ende aten die/ Dat in langhe nist ghe fhiet en wag/ Den bumek bol / Daer bu kokende pap uan meel en water / in plact fe uan beoot/foo dat fi mu goedta mots maren. Deg namiodacoly gin=

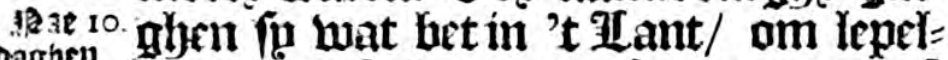

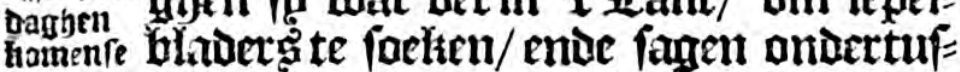

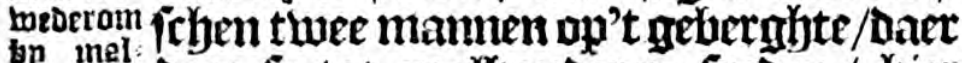
hanberen. Dons fis tot malkanderen fouden / bier maet meer bolckit moonen/ maer keer = Den/fonder meer arfyt daer op te flaen/ nae of fithunt toe. De twee mammen/ zínue ban bare maet unt be andere folute/quamen den berth af na de

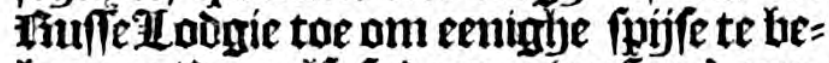
homen/Dam alfófe daer onber/iens aenquamen/ende geen qeit by yaer fadien fon hasdenfe boos baet genomen een paer bzoeften unt te fehieten (die fo wel twee of te dzie ober een hadoen ) om de

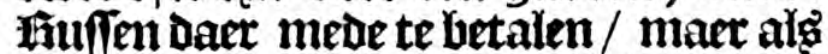
for begonnen te naderen/Fauenfé d'ander fefunt leggen/en werden malfanderen

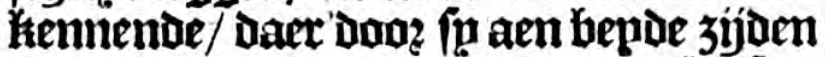
feer verbilijt / mallanderen ombelfen= de / en met marfiandeten wat regeten belbende/oginchende ban den klaren als in den fihín boaz-kn đeulen loout. De $=$ ie maet get haer fobute haoden in worly meerier fluarintsent geweeft / ads d'andere daer wu ban berthaelt heblen/ dancliten daetom gefamentlít den nae: den Gadt / Dat bu haer fan genadelick glyefpaent / ende noch mederom by mallianderen gebracht hadde. Ten 22.quamen D'antuere maet met de back ap de folyeut/Daer Donz ppalle getijek wet berblíjot waren / ende betwillingen boen de Hock van de fiufen dat hip haer em

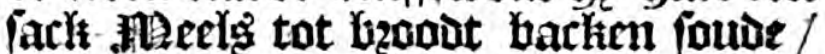
't welelz hp dede: Indidoelertijot de Dif: folyerge unt der Eee komenue horbt de Srhipuer van baer vier Tabeljaumen / jie ju te buex deden / ende alş fp mu aber maeltijot waten/ quam de buerfte vall de suifen by baex / ellde fiente dat fu Luepnigh bzoout hadom / gaf baer een bzoot ; Sp noouen hem om met haer te eten / bact wilde daer niet toe luerfaen / vernitg het bare bafteldaugh was / ente de onfe entige boter of t wet ober de Bifets ge fuolten hadien: Ila men konoe nict

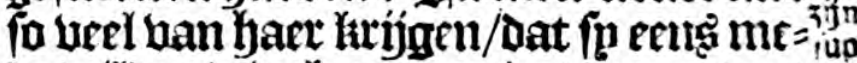
de wilom beincken / on dat be nap wattier

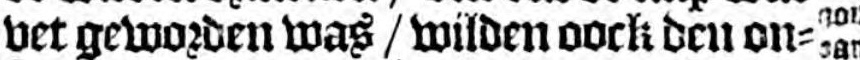
fen bate napyen niet leenen om unt teba

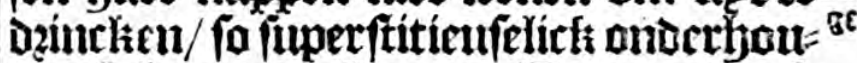
den fu hare bafteldagen. Den 23. Geeft de serfupper den Guerften ban de thif: fen een goeden Dźinck-penning gegeben/ ende den ltock betafit ban jijn moente in't backen/ daer hall fo ben bende feer bedanckten / ente de tluee febunten jijn op beil abont omtrent wefter-Gon ban baex t' zepl gegaen tegen't fooghfte wa= ter. Den 24. amtrent onfter san zín ju bp de feven Eplanden geltamen/ Daer fu beel Biffechere bonden die fu bzaeghoen nae Kool oft Kilduyn, mbe wefen hacx al weft aen / 't welch fị mede fo verfton= den / wierpen baer oork een Cabbeljaut tae / die de onfe boaz den yoeden bonatgangh die fu badoen / baer niet en kion= Den betalen / maer bedancktenfe / feev verwondert zínoe aber baere beleefts 


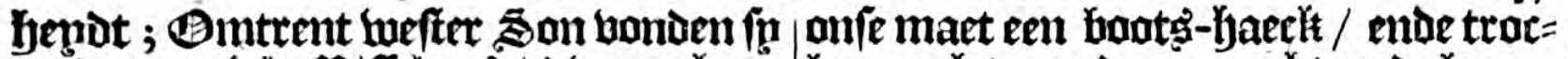
weder eninge Biffebers / Die nae haer toe roepdoen / ende bzaeghoen waer haer

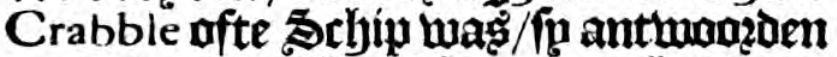

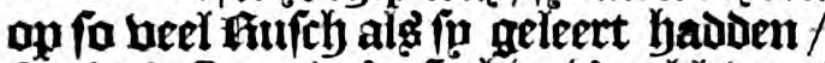
Crabble Propal, 't Sithip is gebleben/ waer op de Biffetyers bederom ríepen/ Cool Brabante Crabble, Daer unt de onfe verftonden/ Datter tot Cola eenighe alze= Dertant fetbe Brthepen waren.

Den 25. Dita hebiben fu Kilduyn am=

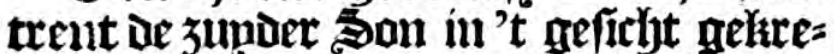
ten / enoe zín amtrent $\%$. Z. MB. Fon aen't weft-eynue de felwen elplant

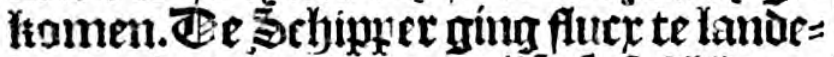
maert in / mobe bouter biff oft feg hlenme hutu/teng/Daer Lappen in woonden/die

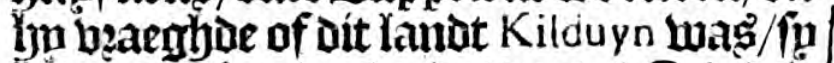
Liedunuen jae / ende Datter tot Cola Die Brabintifche Crabblen lagent maer dat de there dien Dagly fouton af-baren. TRe

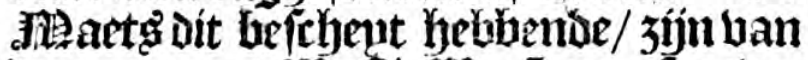
Darr ointrent 13. W. TB. Son af-neba= ker / in menninge zijnise naer $W$ arthuys te 3 enyten/lliaer in 't 3 enlen zínde / gaf or wint fo ge herloig ap/Dat fo't ober nactht niet en do:fen in ₹ee fouben / ende na= men yaer courg achter twee fitlippen na 't Il andet tae: Baer bonden fon weder een fileun butghen faen met deze mamen

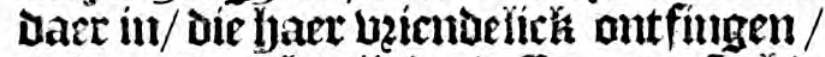

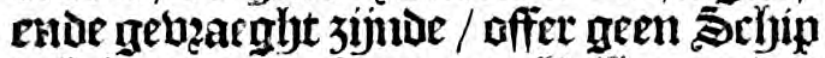
te betiomen way om waer follant te ba= $\mathrm{ren} /$ ant woosoen mede fo $\mathrm{o}^{3}$ ander il ap= pen gedaen hadoen/ Datter dzie Selhepen lagen daer baun de twee dien dagt menn=

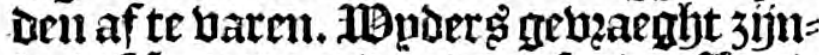
de/ of f́n met een ban de onfe aber li ano nae Cool mildent gaen/men fouldet baer rerlick toonen/ont

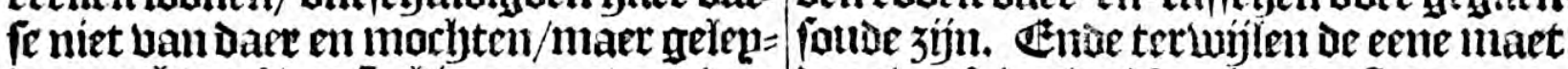

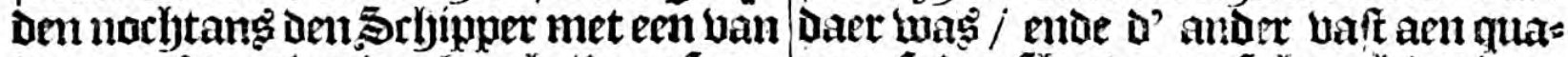

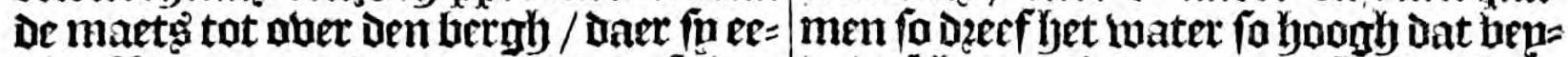
nige IL aypen bonden / Luaer ban fn den de de fibututen in't Luater wefimeten wer=

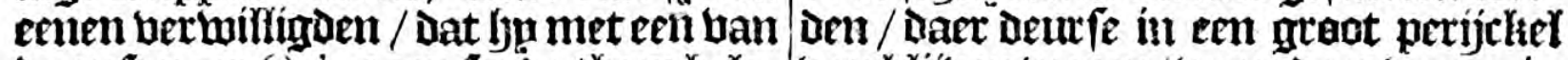
ae omfe naer Cola gaen foube/ hem belo= baul bilijuen waren/ want daer waten in

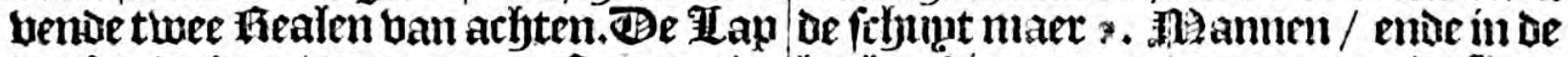

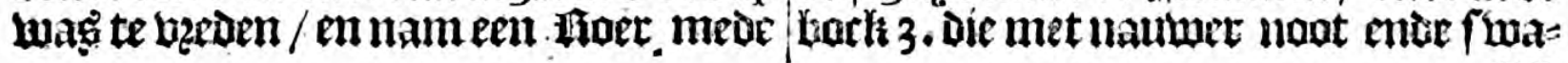


48 Derde Schip-vaert van Willem Barentiz.

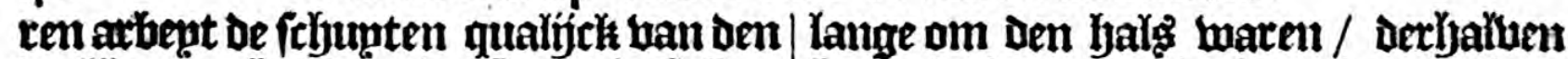
wall handen youdon / Dat fe niet in ftuken gefmeten werden. IDp dat fiende / wa: ren feer beducht ende kanden baer niet helpen/ eben wel norty Gadt Danckende Dat hy ong eerft fa berde aebzacht hadare dat men eben buel baozet fouden moghen homen / oft luy de felbuuten berlooren / als' Dachende nacht getweldigh / Daer deur unp groot ellendt ende angemack leden / want wo werden deur nat/ende Konden ong niet deckten nach daet boa: befther=men / maer die in de frbupten warenle= Den noch den meeften noat/ Datfe in fulte= ften weet ende reglyen aen de leeger wal berblijben maeften.

Den 28. Ilugufti maft tamelijeft me= Dete / alfo Dat wap de fetyunten wederom op't 'Jant Yaet den/oun die refte bau't goet Daer unt te loffen / om dat períćckel te ontgaen daer de fehtunten in nhetweef Yjadien/ om dat de wint noch ftijf uuten

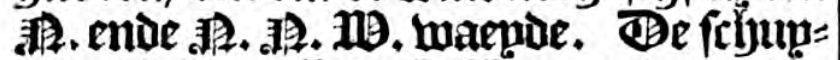

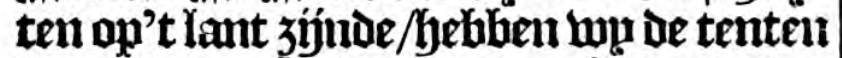
Daer ober gefpaimen am ong daer onder te bebelwen /want het $\mathfrak{w a g h ~ n o c l y ~ a l ~ e b e n ~}$ miftiry / regenachtigly weder/qroot ber= Laugen hebbende tijoinge te krijgen ban

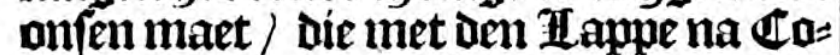
lagegaen was/om tijoinge te bernemen

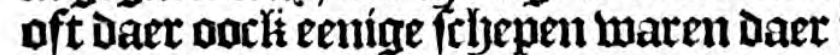
mede men nae Molfandt mochte varen. In islertijt dat wu daet lagben / Jaelde = men dadbelificx te lantot-waert in nock ban de bilaen we beffen ende braembefien die wy aten / ende ong gront gaet deden.

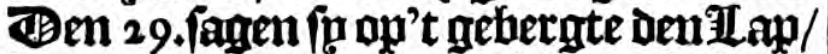
Die met een ban de maet naer Cola ge= gaen lwa: / weder aen Kamen/ Doch fon = Der fín met-gefefle / dat lyaer wonder maf/ende maxen Daer in beduclyt/ maer de ILap bp haer Kamende / gaf den

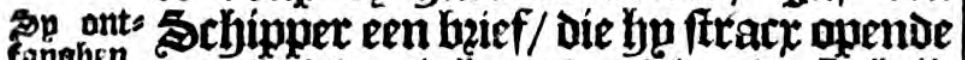

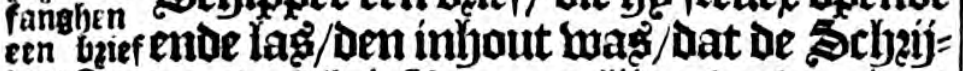

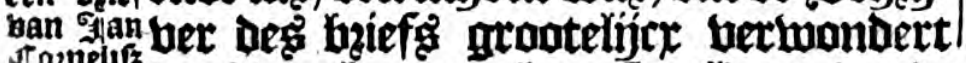

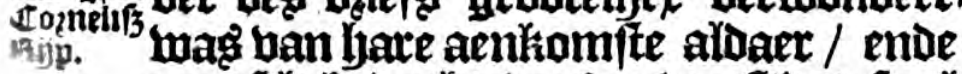
hu nu des te meer in lyacr faimfte ber= blijt wag/ ende foude terftont met allen noot dzuft van eten ende dzanken bo ljaer fomen/ondertepckent/ bu mp lan Cornelifz Rijp. TBooz defe blijoe týpingtye bebhen fu den Ilappe zijn belouf be pen= ningen getreben / ende daet-en-boben noch eenige hiterderen (ald boxen / kou= fen ende atoers) gefetjoncken / dat bu lyeel op fijn ollantich toe-gemaecht

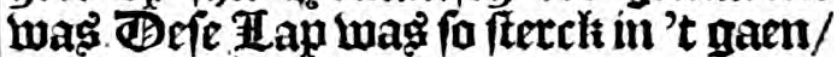
dat lyet monder om feggen iz/ Lwant in't unt-gaen/fá zijn rewf-gefel fende/waren= fe met een fijbe, gamgly twee daghen en twee nathten ap de hurgly/ eer fun te Cola quamen / enve nufn't 't wederom kamen was hy maer enen etmael ander weghe / Dat immers de rechte belft febeefoe: - Baer buer de maet tot malltamderen

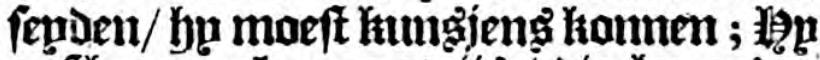
gaf haer oock een patrýs / die yn anoer wene geftyoten yador.

Win 30. waren [p noeb beftammert/ wie die Jan Cornelifiz. was die aen ljaet gefebzeben fadod. Onder ander werter wefent/oft niet wel die Jan Cornelifz we= ien mocht die met lyaer in een compag: nie gevaren hadde/ bocty ontgabent haet wever / om dat fu immers poo feer wan= baopten ban fín leben/alg' lyp ban't tya= $\mathfrak{r e} /$ mequende dat hy 't noch nuader ge= haot hadoe dan $3 \mathfrak{p} /$ ende aber lange ber $=$ gaen was. In't epnde bont de schipper nach onder zign bezieben de ham dt ban die Ian Cornelilz. die met hem gebaren hads de/ enae bevonden doen dat bet de felbe

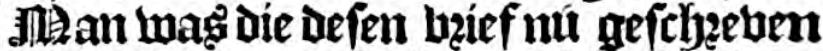
hadoe / daerom fímmers foo feer ber= bljot waten ban fijn befoudeniffe / alta

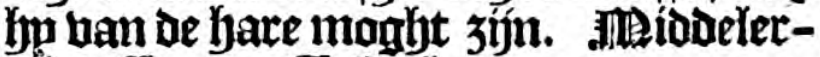
trjot iffer een \$olle komen aen raenen Daer lan Cornelifz felben in mas'/met de Mla an bu baer untgefonden: Tlaer ont= fingen pu malfanderen aen landt met mooter bitíf f́hap / als bf elck aen weder 3 jijoe den mineren wan der doot wederom

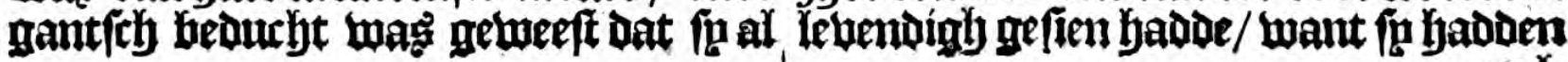


Om't Noorden na China.
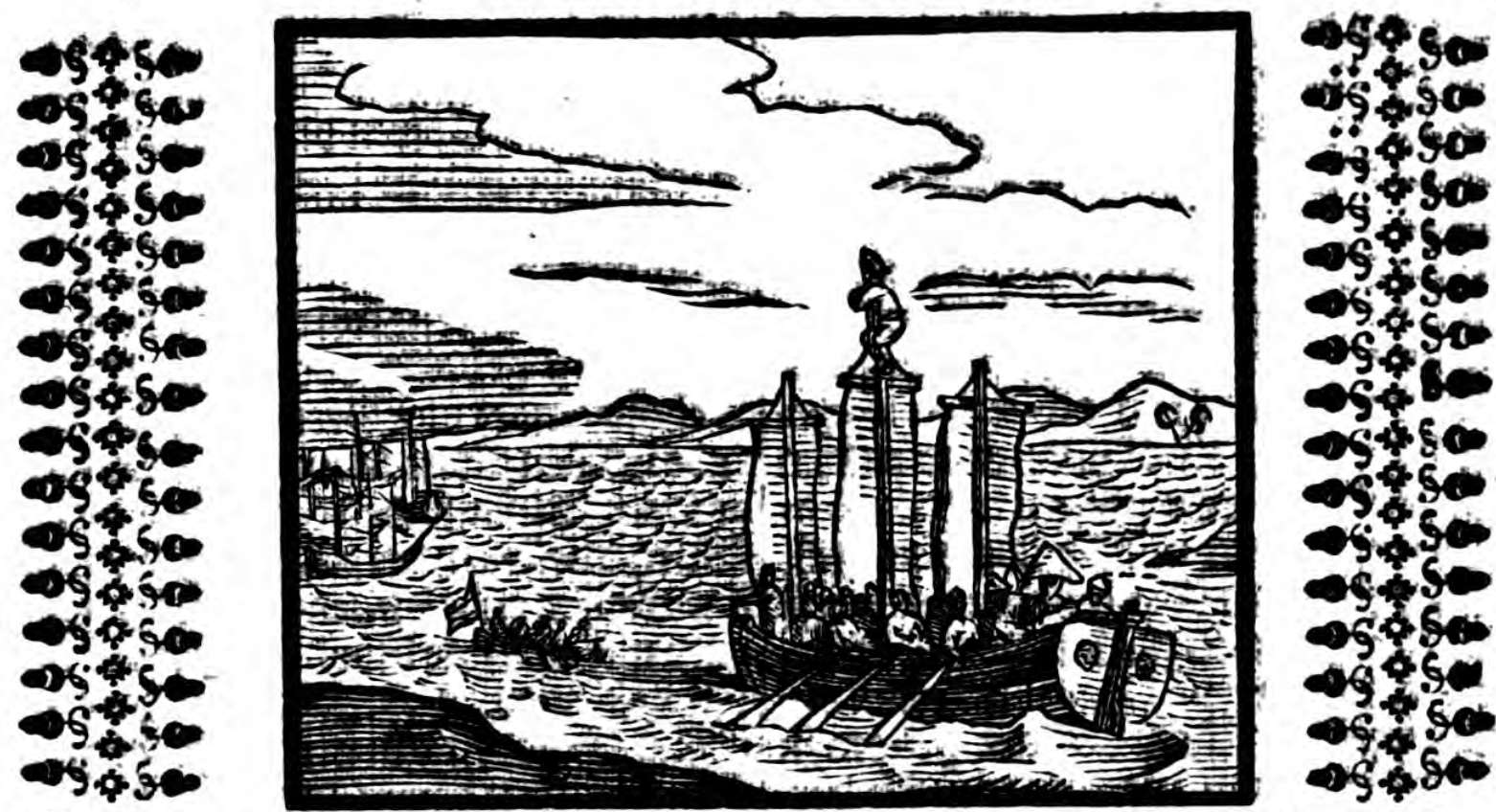

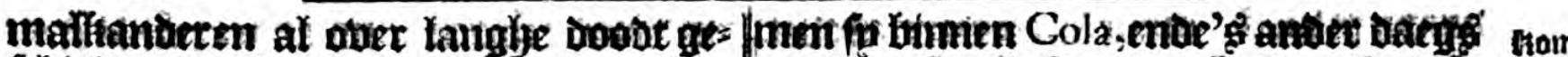

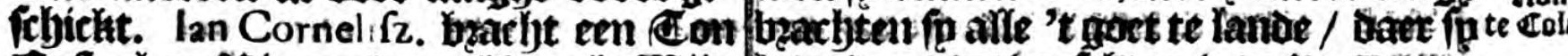

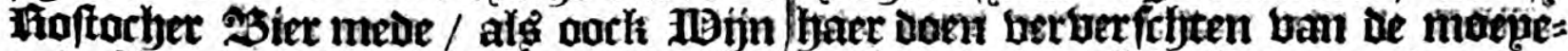

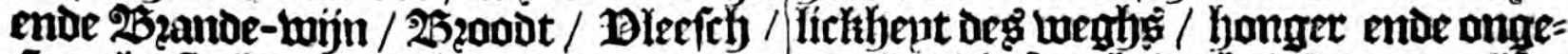

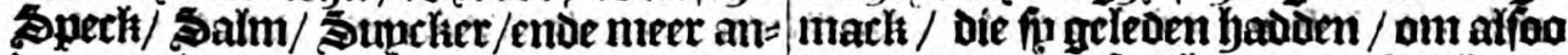
der probiande / Dat de maet feer ber= Lederom tot gefonthent ende fterchite te

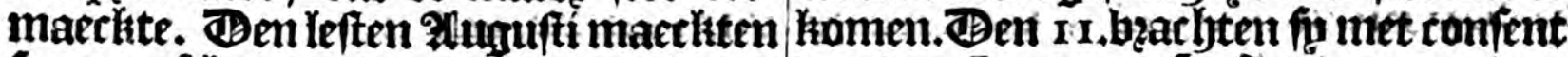

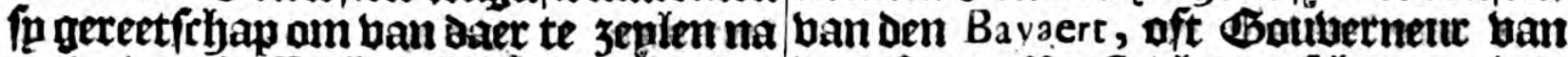

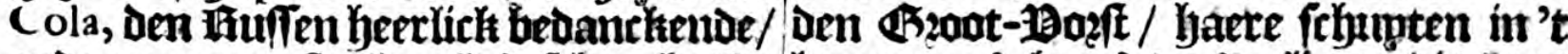

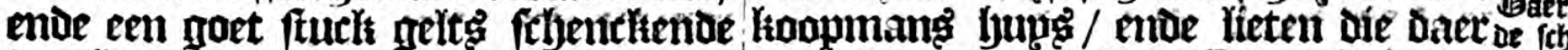

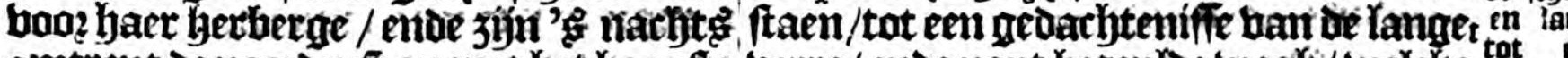

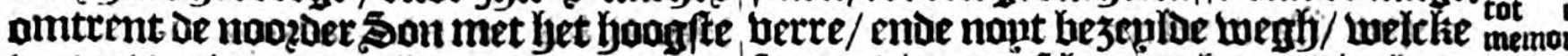
water ban daer gezenilt.

fo met die open fehunten bu na vier bon=

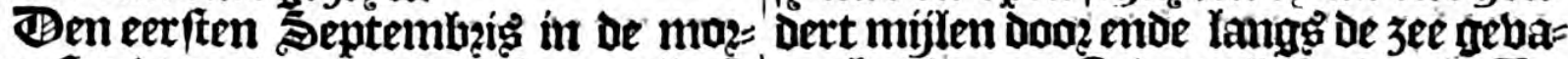
gen-ftonot omtrent oofter Son / zín in ren badoen tot Cola toe/ Daier ban de Fn=

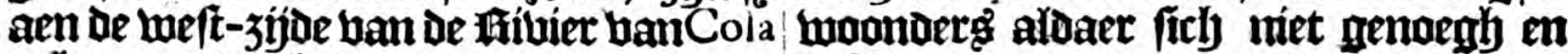
geltamen / 3eplende ende roepende boagt fanden bertwonderen

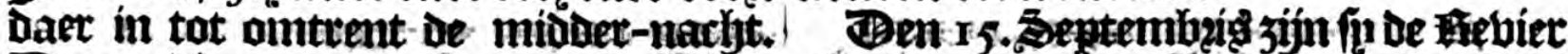

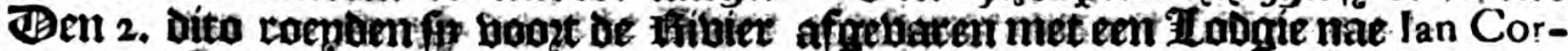

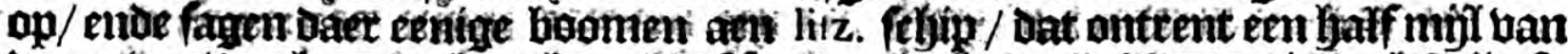

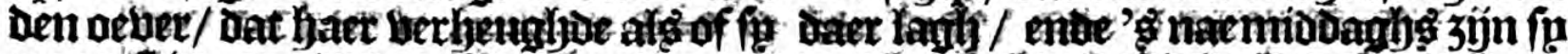

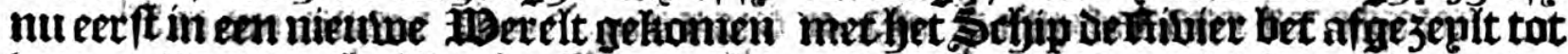

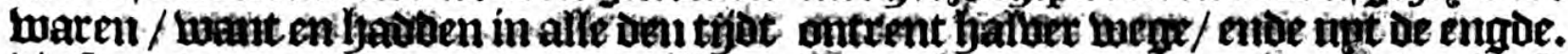

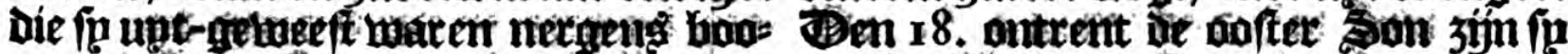

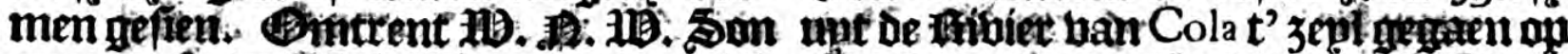

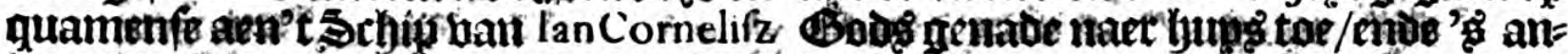

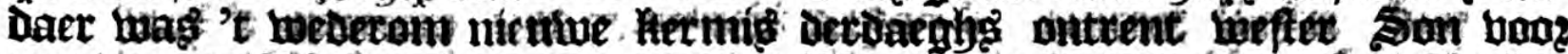

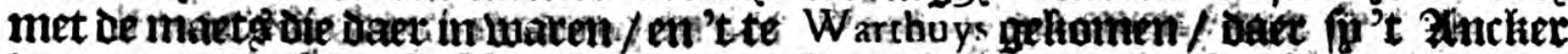
boorentmet of fellue Srfjupier mede wa fieten baffen/om dat lan Cornelifz. Daex ren getweeft op de reps ban't boolteden nocly gaet maeft imemen/ende bleben=

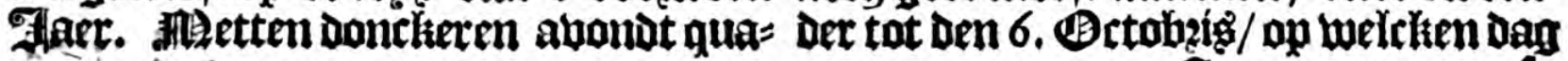




\section{so Derde Schip-vaert van Willem Barentfż. om't N. na China

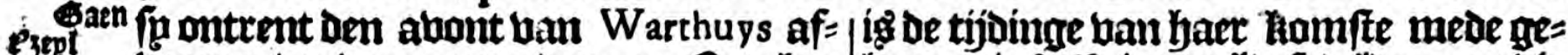

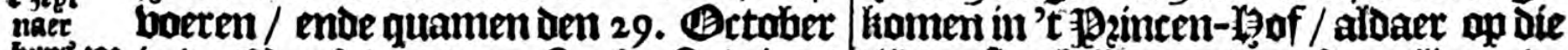

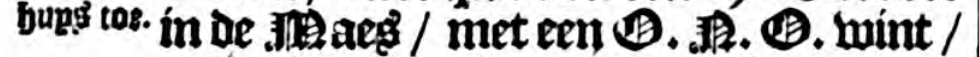

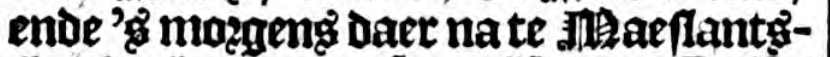 fungs te lanoe / repfoen alfídooz Delft/ ben 1 aeglyende 1 arertem/ende nuamen

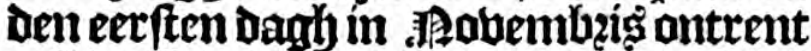

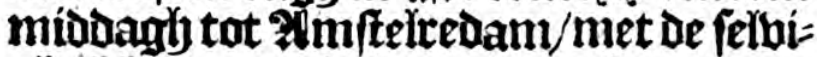 ghe hleederen die fin op Nova Sembla ge= Dzagen hadoen/ met bonte mutien ban witte Baffen / ende gingen in tat Pieter Haffelaer, die een ban de 2 Selwint-lyet= $=$ berge der ftad ban 2 Imfteltedam getweeft wag/ tot untruftingthe luan de twee fche= pen / te weten lan Cornelifz. ende on=

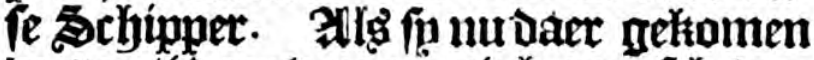 waten/(daer van menigh menfth ber= wondert waga / want men haer al ober lange doot gerekenthadde:) en dat ge

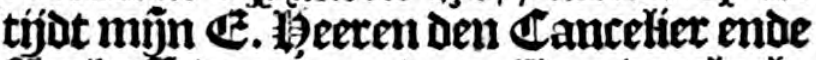 Wmbafladeur ban den afler-boozluch =

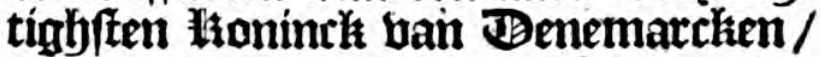 13aoztwegen/Batten ende IBenden aber Tilafel fat. Derbalben sijn fin teef fonot derwaert allyaelt dong mijn Erbout/ende tiwee foeren van der ftad/ ende lyebhen aldaer boos den boomaem= de 2burgemefteren bertellinge gedaen ban haer repfen ende weder-baren/ ende zijn daer nae elch nae humg gegaen die daet t' lyug hoozden / maer Dié daer niet $t^{\prime}$ huyg boozden sijn in een 1 (erberge ge: ftelt fammige dagen/tat dat fón baer gelt ontfangen lyadoen/ende daer nae iagelctit ijng weenth geteyft / met gaet genae=

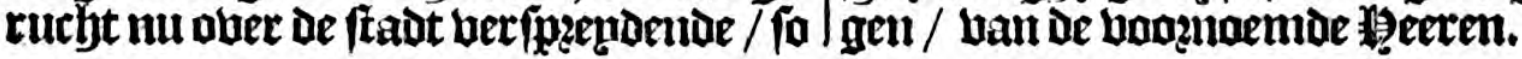

De namen van de geene dievan defe Reyfe weder gekomen zïn. Iacob Heemskerck, Commijs Ian Hillebrantfz. ende Sichipper. $\mathrm{Mr}$. Hans Vos, Barbicr. Laurens Willemfz. Pieter Cornelifz. Pieter Pieterfz. Vos. Iacob Ianfz. Sterrenburgh. Ian van Buyfen. Gerrit de Veer. Leenaert Hendrickfz. Iacob Ianfz. Hoogh wout. Iacob Evertfz.

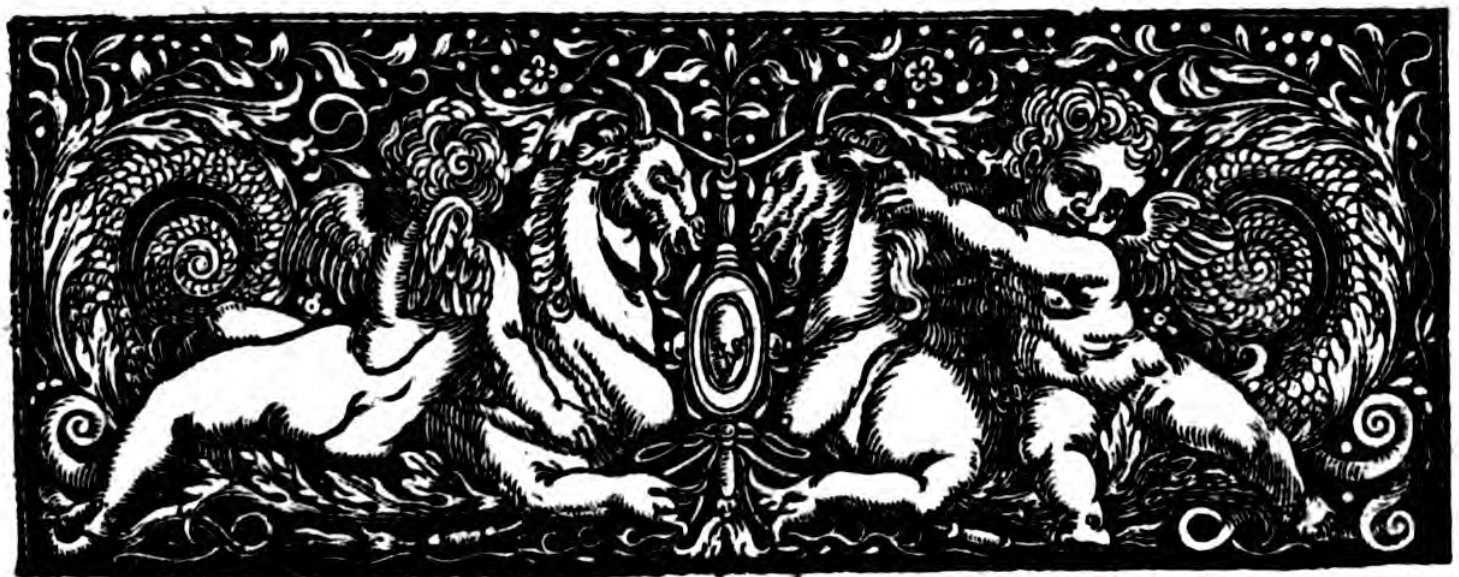


Fol: 5 í.

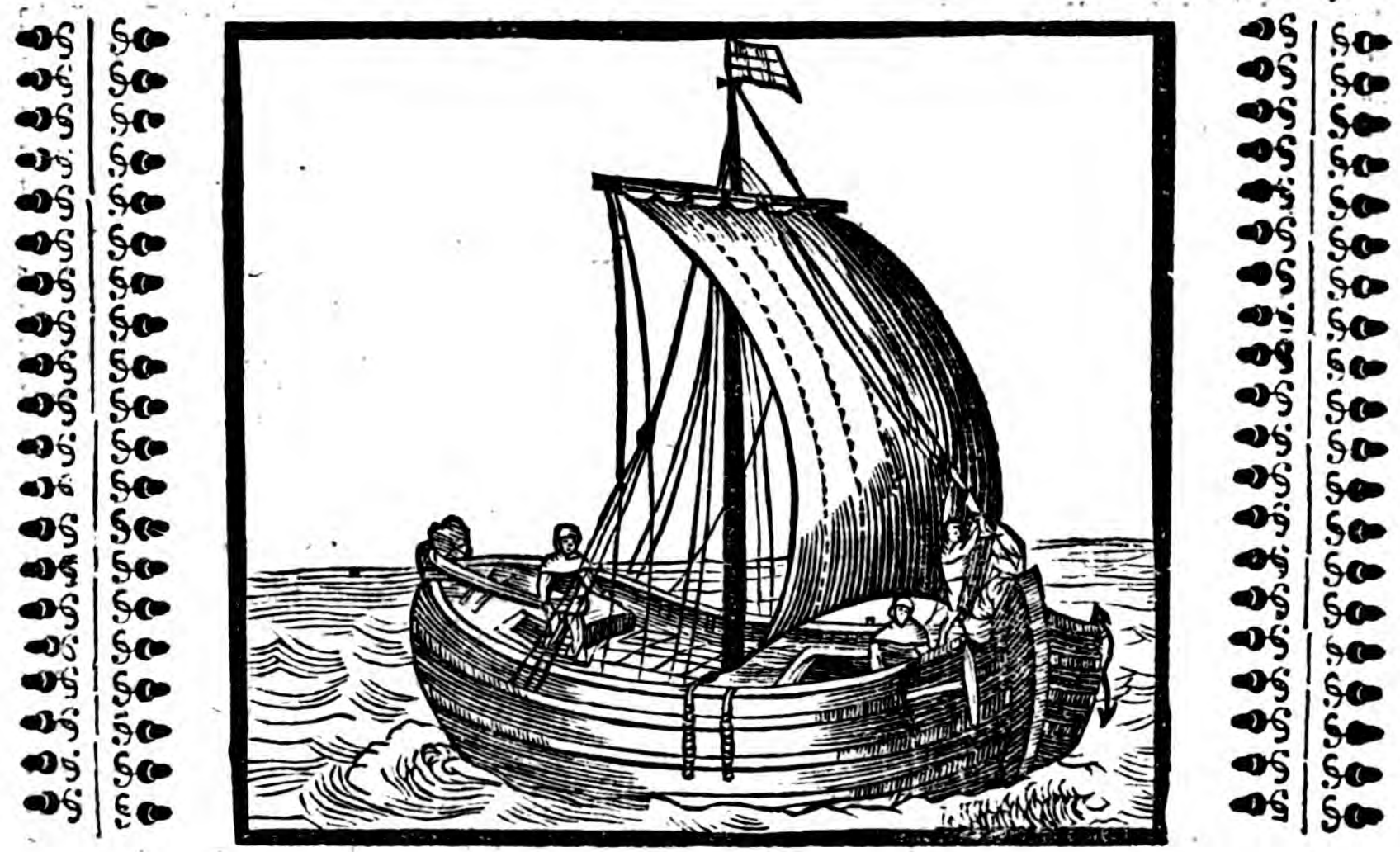

\section{Journael van Herry Hutfon,}

\section{Gedaen in den Iare 1609.}

\section{Op het believen van de Bewint-hebberen van de Ooft- Indifche Geoctroyeerde Compagnie uytgefonden, om de paffagie by het Noort-ooften, ofte Noort-weften om naer China te foe- ken, met een Vlie-boot, wel'voorfien, ende met 20 mannen foEngel- fche, als Nederl inders bemant, gelijk uyt het volgende te fien is.}

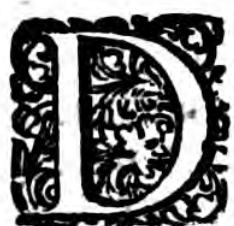

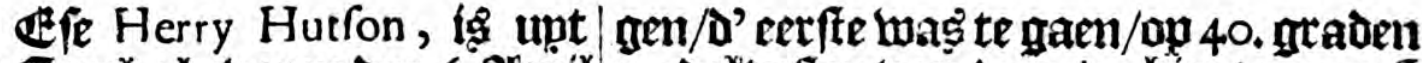

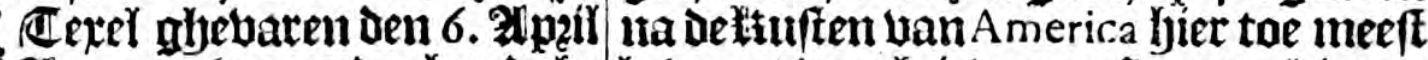

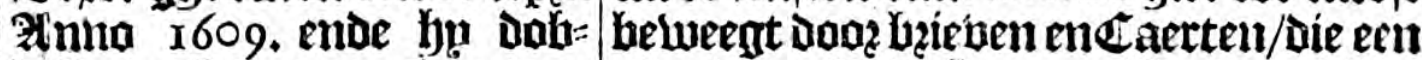
betoe de Cabo yan a

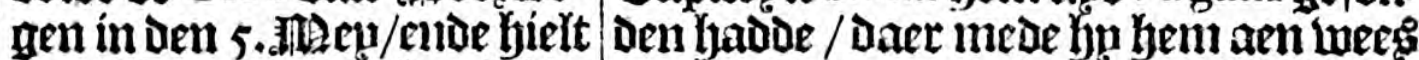

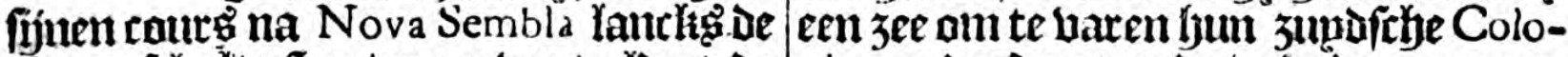

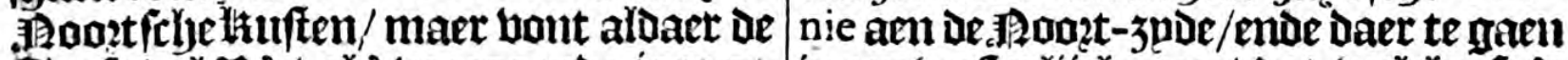

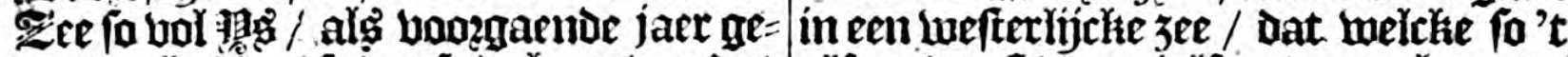
bonden hadoe / fo dat fu de bape ban dat alfa getweeft Lare (arfa erbarent hept tot

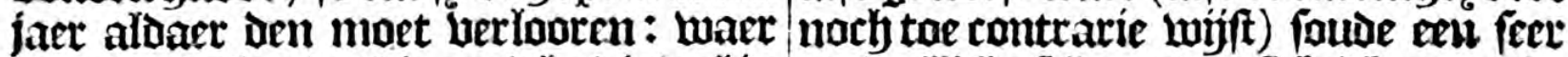

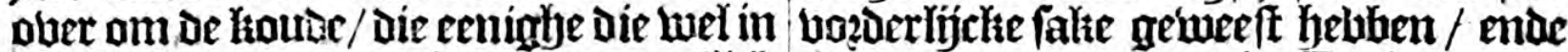

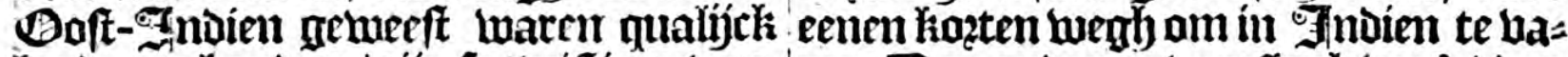

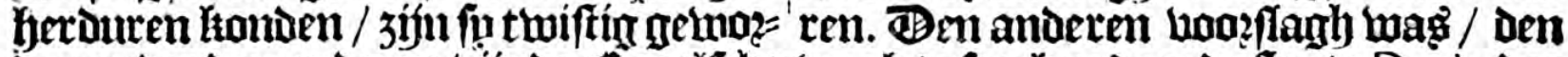

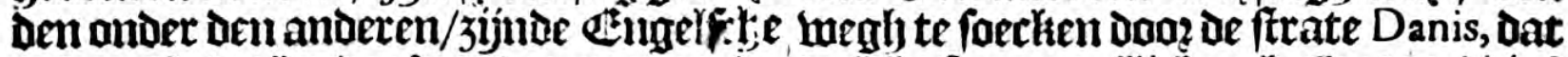

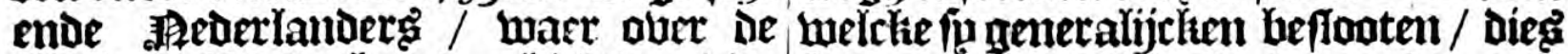

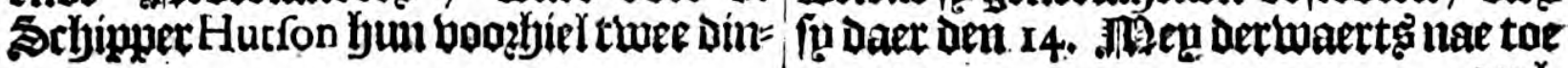


setitoen / en quamen met goeden wint ben leften an we aen't Eplant ban Faro,

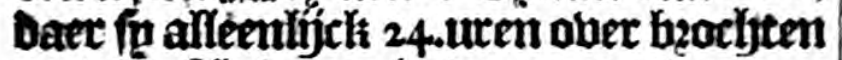
met berfh water in te nemen / vertree henoe/boeren totten 18 . Flutij tot op se Atuftem bam Nova Francia op 44.graven/ baer fo moeften intopen/om cenen nieu= tren bonz-maft te beltomen / ben baren betlouren lyebbende / die fp daec bomien enve opfteloen / pu bonden ore plact é be meaem om Gabbeljaente banten/alg oocli m traffique ba goede gupom ente Belfien ofte ABeneringe/wat abaex om hlepme dingen te behomen wag/ maet lyet Sebju - bolek leef de qualick met het 1 aut-bolek/ Dingen met getweit nemende / waer ouer in twiftighender Den amoeren werden/ be Engelíthe bze: fende dat in bermant waren en de beek $=$ fte/ende daetonme bzef den bobzer te berfoecken / aldits febepden fu ban daex Den 26. Fluth / ende bielden de Zer tat ben 3 iumuti / enve quamen un I anot op 42. meaden: 1Ban daer boerm fo boos= ber totten I 2. OAumufti / quamen weder bn Tamot op de I atitude bain 37. D2p? Eerr quart/ ban daet hielden bu't Ilant / tot Eution bat fin quamen op 40. en òn quart ma= jeeft

Den / aldaer fo vonoen eenen goeven in ganck tuffechen twee hou fuen / entue boe= ren daer inme den 12 . September / een alfoo feljoonen thibiere alg men hande binden / wijot ende diepe / ende graeden Zuteter-gront / epndetick quamen op oe Tatititue bam twee-en beertight mi= nuten met fun groot sobix. Ban haer Schip-boout boer boonter in de ribiere. bomien fo laloectente weetbaer volel / maer bimen in't unterfte banden bien= Delifotit ende beleeft bolck / Die beel hif-

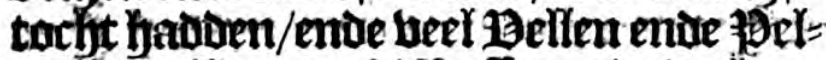

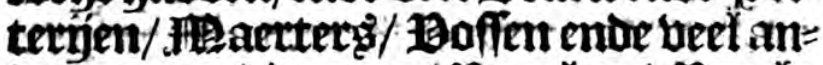

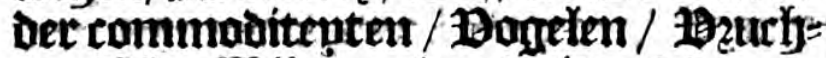
ten/felve Mibinoanuen/ witte chore roo= be/ende thamblont beteef delfiction.met:

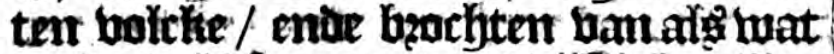
meite / atgi pin nu ontrent bijftigh mijlen

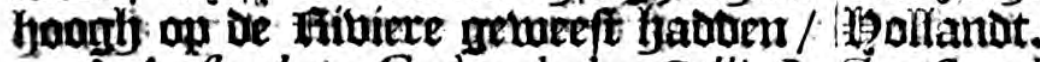
t. Arnfterdain, Gedruckt by Gillis Ioost.n Saeghman, in de Nieuwe-ftraet. 3jin weder gelteert den bierden(B) tobrig ende bum weder ter Zee begetuen: baer hadoen meet loumen untuerecht mos den/ badoe daer gaede wille in't sochipbolch greweeft/ende mede't reboech ban cenige nootouft futches niet beethinuert. In Zee bebibenbum beraedflaedht/en= de waeten ban berfethenden opinien / De - Bnder-fithipuer een faederlander wat barmepninge / op Terra Nova te gaen vecwinteren/ ende de zoont-wefte yaf = fanir uan Danis te Dongfoekten/Daer Lag or Erbipper Hut Ton teghen / Die begef de fijn gemutineert bolck / on dat fo by wiflen hadoen gedzenglits / ende mede / Dooz ar houde des binters / hun gelsed fousen berteeren / ende dan moeten kee= reil: Deet ban't bolch teer moe fiectie= lícck / niemanot nochtang pparf ban t' lyups na follant te baxen / Iam Den Schuper 't weirk naderkanot achter= Dencken gaf / dieg lyn boazfoegh na 量r= lant te baren enve te berwinteren / Dat ip alle taeftemben / dan ten leften jọn in Eirgelant tot Dertmouth Den 7. 120= bember gheltomen / bau waer fu haer

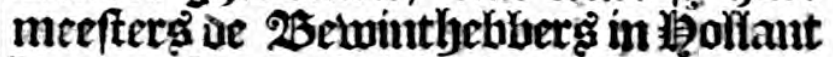
haer revfe bertwittight; boos-flagh Doen=

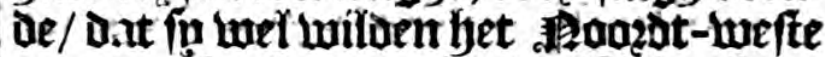
gaen uerioecken / miots bifthien bon= Dent gutben in gelide meer in noatoguft te beftesen / beniffeng den loon / ende dat pu in 't Schip alteede hadoen/ Dies

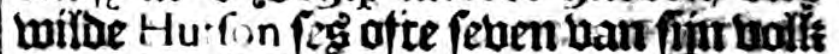

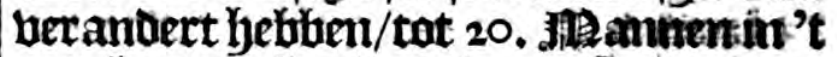
netal op matienoe / Ece. en fouben ban Dertmouth t' zenl gaen ontrent den

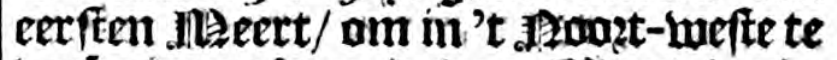
wriar tenen't epnde ban flicert / ende dare is natent wam alpgil ende balf for ober te brengen met 10 albififyen te van= gen en 23 reften te Doaden ontrent bet Eplamot van Panar, ende dan nae fyet

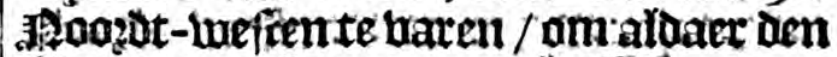
tijot obet te bzenten tot half Septem=

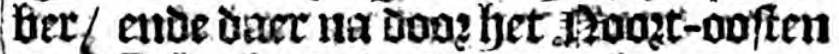

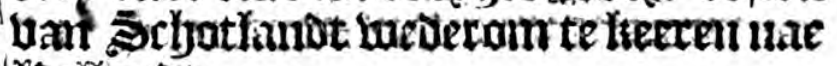
Holfanot. 

1

$$
\text { - }
$$


. 
- 


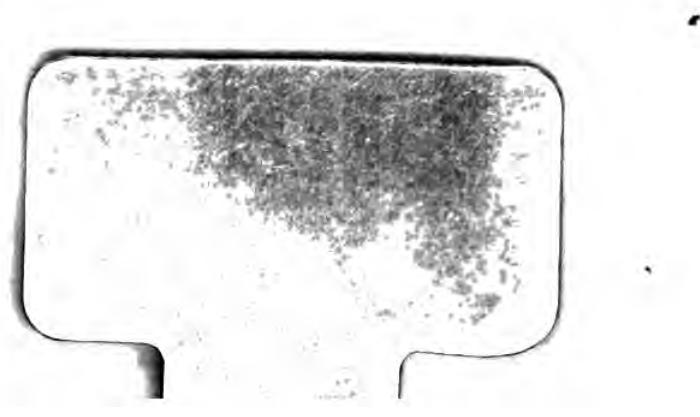




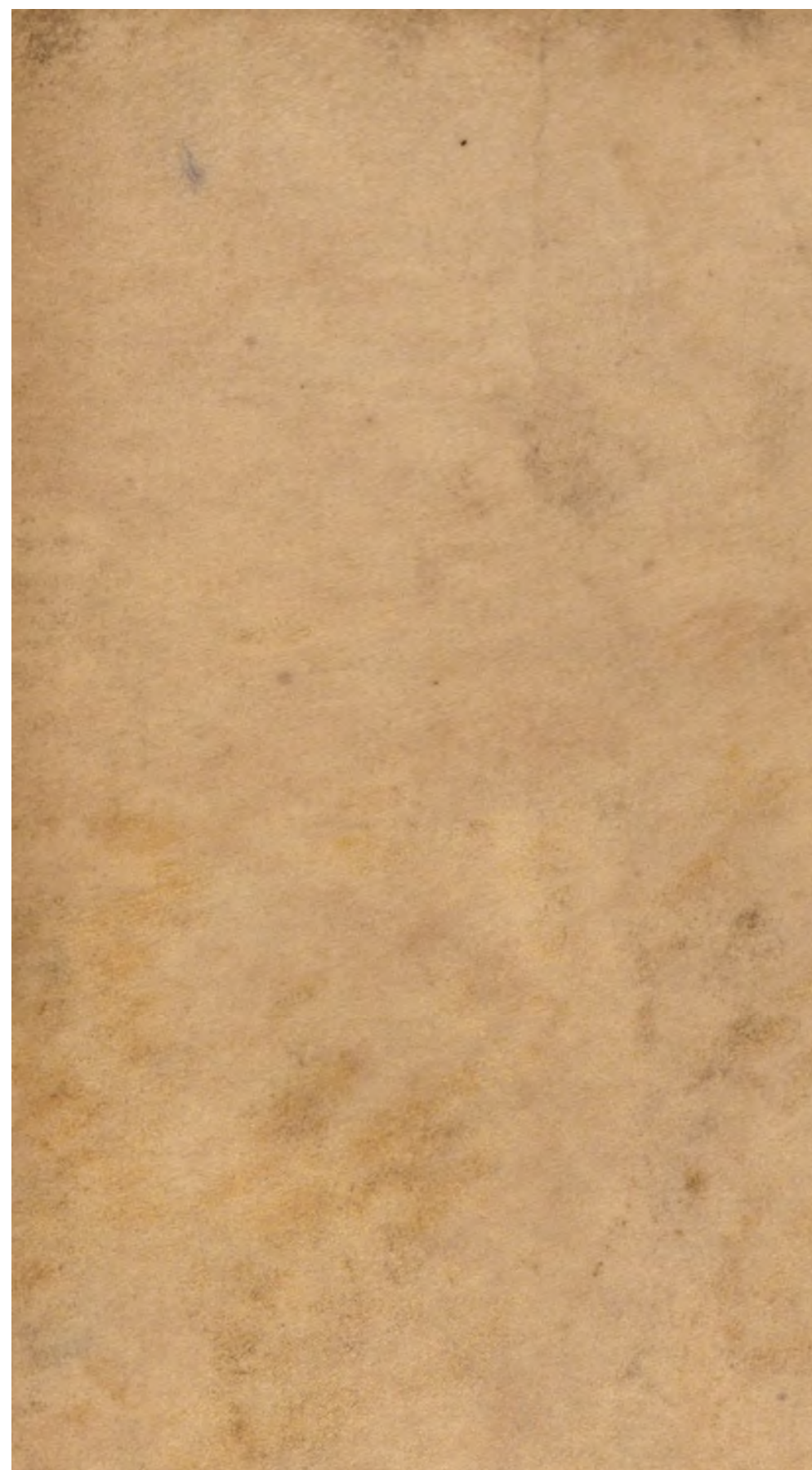

Ses 